\title{
Beta Function and Anomaly of the Fermi Surface for a d = 1 System of Interacting Fermions in a Periodic Potential
}

\author{
F. Bonetto ${ }^{1}$, V. Mastropietro ${ }^{2}$ \\ ${ }^{1}$ Dipartimento di Matematica, Università di Roma, 00135 Roma, Italia \\ 2 Dipartimento di Matematica, Università di Tor Vergata, 00133 Roma, Italia
}

Received: 25 April 1994

\begin{abstract}
We derive a perturbation theory, based on the renormalization group, for the Fermi surface of a one dimensional system of fermions in a periodic potential interacting via a short range, spin independent potential. The infrared problem is studied by writing the Schwinger functions in terms of running couplings. Their flow is described by a Beta function, whose existence and analyticity as a function of the running couplings is proved. If the fermions are spinless we prove that the Beta function is vanishing and the renormalization flow is bounded for any small interaction. If the fermions are spinning the Beta function is not vanishing but, if the conduction band is not filled or half filled and the interaction is repulsive, it is possible again to control the flow proving the partial asymptotic freedom of the theory. This is done showing that the Beta function is partially vanishing using the exact solution of the Mattis model, which is the spin analogue of the Luttinger model. In both these cases Schwinger functions are anomalous so that the system is a "Lutttinger liquid." Our results extend the work in [B.G.P.S], where neither spin nor periodic potential were considered; an explicit proof of some technical results used but not explicitly proved there is also provided.
\end{abstract}

\section{Introduction and Statement of the Results}

We study by renormalization group techniques the analyticity properties of the Beta function and the behaviour of the pair Schwinger function for momenta near the Fermi surface for a one dimensional system of $n$ fermions moving in a common periodic field $-\partial_{\vec{x}} U(\vec{x})$ and interacting by a short range pair potential. We consider both spinless $\sigma=0$ or spinning fermions $\sigma= \pm 1 / 2$. The recent interest about interacting electrons in a periodic potential [D.M., Sh.] motivates our study. The one dimensional hamiltonian is

$$
\begin{gathered}
H=T+\lambda V, \\
T=\sum_{\sigma} \int_{-L / 2}^{L / 2} d \vec{x} \psi_{\vec{x}, \sigma}^{+}\left(-\frac{\partial^{2}}{2 m}+U(\vec{x})-\mu\right) \psi_{\vec{x}, \sigma}^{-},
\end{gathered}
$$




$$
V=\sum_{\sigma, \sigma^{\prime}} \int_{-L / 2}^{L / 2} d \vec{x} d \vec{y} v(\vec{x}-\vec{y})\left(\psi_{\vec{x}, \sigma}^{+} \psi_{\vec{x}, \sigma}^{-}\right)\left(\psi_{\vec{y}, \sigma^{\prime}}^{+}, \psi_{\vec{y}, \sigma^{\prime}}^{-}\right),
$$

where $\psi_{\vec{x}, \sigma}^{ \pm}$are creation or annihilation field operators with spin $\sigma$ on the Fock space of a fermion system confined in a box $(-L / 2, L / 2)$ with periodic boundary conditions and at zero temperature, $m>0$ is the electron mass, $\mu$ is the chemical potential, $U(\vec{x})=U(\vec{x}+a)$ is a $C^{\infty}$-smooth periodic potential, which for simplicity will be assumed even $U(\vec{x})=U(-\vec{x})$; $a$ is the lattice spacing, $\lambda v(\vec{r})$ is the spinindependent, electron-electron, interaction, supposed to be even in $\vec{r}$, bounded $C^{\infty}$ smooth and $p_{0}^{-1}$ is the interaction range. Clearly we must have $L=N a, N$ integer, and we choose a system of units in which $\hbar=1$.

It is well known (see for example [Ko.,T.P.]) that it is possible to find two functions $\varepsilon(\vec{k})$ and $\phi(\vec{k}, \vec{x})$ defined for complex $\vec{k}$ and satisfying the equation:

$$
-\phi(\vec{k}, \vec{x})^{\prime \prime}+U(\vec{x}) \phi(\vec{k}, \vec{x})=\varepsilon(\vec{k}) \phi(\vec{k}, \vec{x})
$$

with $\phi(\vec{k}, \vec{x}+a)=e^{i \vec{k} a} \phi(\vec{k}, \vec{x})$. Two such functions are holomorphic everywhere except on the vertical segments joining the point $\vec{k}_{n}=(n+1) \pi / a+i h_{n}$ with the point $\vec{k}_{n}^{*}$, and the point $-\vec{k}_{n}^{*}$ with the point $-\vec{k}_{n}$, where $h_{n}, n=0,1,2, \ldots$ is a sequence of real numbers such that $\lim _{n \rightarrow \infty} h_{n}=0$. On the cuts the functions present a jump discontinuity and near the point $\vec{k}_{n}$ we have

$$
\varepsilon(\vec{k})=\varepsilon_{n}+c_{n}\left(\vec{k}-\vec{k}_{n}\right)^{1 / 2}+o_{n}\left[\left(\vec{k}-\vec{k}_{n}\right)^{1 / 2}\right],
$$

where $c_{n}$ is a bounded constant, $\lim _{n \rightarrow \infty} 2 m a^{2}(n+1)^{-2} \pi^{-2} \varepsilon_{n}=1$, and

$$
\phi(\vec{x}, \vec{k})=\frac{D(\vec{x})}{\left(\vec{k}-\vec{k}_{n}\right)^{1 / 4}}\left(1+C(\vec{x})\left(\vec{k}-\vec{k}_{n}\right)^{1 / 2}+o_{n}\left[\left(\vec{k}-\vec{k}_{n}\right)^{1 / 2}\right],\right.
$$

where $C(\vec{x})$ and $D(\vec{x})$ are holomorphic functions. The symmetry of $U(\vec{x})$ clearly implies analogous formulas for $\vec{k}_{n}^{*},-\vec{k}_{n}$ and $-\vec{k}_{n}^{*}$. Finally it is possible to fix the mean of $U(\vec{x})$ so that $\varepsilon(0)=0$.

For $\vec{k}$ real, $\phi(\vec{k}, \vec{x})$ are called "Bloch waves" and $\varepsilon(\vec{k})$ is the "dispersion relation." The functions $\varepsilon_{n}(\vec{k})$ and $\phi_{n}(\vec{k}, \vec{x})$, where $n$ is the "band index," are $\varepsilon$ and $\phi$ restricted to the segments $(n \pi / a,(n+1) \pi / a]$ and $[-(n+1) \pi / a,-n \pi / a)$. The periodic boundary conditions imply that $\vec{k}=\frac{2 n \pi}{L}$. Physically one defines the Fermi momentum $p_{F}$ so that the ground state energy of the hamiltonian Eq. (1) has the minimum at $n=\frac{2 p_{F} L}{2 \pi}$ when $\mu=\varepsilon\left(p_{F}\right)$, and the Fermi velocity $v_{0}$ is defined as the minimum energy increase by adding a particle to the ground state, divided by $\vec{k}_{0}-p_{F}$, if $\vec{k}_{0}$ is the momentum of the particle added. We require that $p_{F}=\frac{2 \pi}{L}\left(n_{F}+1 / 2\right)$, where $n_{F}$ is an integer (and this of course is possible if $p_{F} a / \pi$ is a positive rational number). It will be easy to see that our results remain valid also without this simplifying condition. The band "containing" the Fermi momentum (i.e. the band such that $\left.\phi\left(p_{F}, \vec{x}\right) \equiv \phi_{n}\left(p_{F}, \vec{x}\right)\right)$ will be called the conduction band.

The creation or annihilation operators $\psi_{\vec{k}, \sigma}^{ \pm}$of a Bloch wave are defined by

$$
\psi_{x, \sigma}^{ \pm}=e^{x_{0} T} \psi_{\vec{x}, \sigma}^{ \pm} e^{-x_{0} T}=\frac{1}{L} \sum_{\vec{k}} e^{ \pm(\varepsilon(\vec{k})-\mu) x_{0}} \phi(\vec{k}, \pm \vec{x}) \psi_{\vec{k}, \sigma}^{ \pm}
$$


The "propagator" of a Bloch wave in a volume $L$ with temperature $\beta^{-1}$ is given by

$$
\begin{aligned}
g(x, y) & =\delta_{\sigma, \sigma^{\prime}} \frac{1}{L \beta} \sum_{k} \frac{e^{-i k_{0}\left(x_{0}-y_{0}\right)}}{-i k_{0}+E(\vec{k})} \phi(\vec{k},-\vec{x}) \phi(\vec{k}, \vec{y}) \\
& \equiv \delta_{\sigma, \sigma^{\prime}} \frac{1}{L \beta} \sum_{k} e^{-i k_{0}\left(x_{0}-y_{0}\right)} \phi(\vec{k},-\vec{x}) \phi(\vec{k}, \vec{y}) g(k)
\end{aligned}
$$

Here $x=\left(x_{0}, \vec{x}\right), k=\left(k_{0}, \vec{k}\right), e^{\imath k_{0} \beta}=-1, e^{l \vec{k} L}=1, E(\vec{k})=\varepsilon(\vec{k})-\mu$. It is important to note that in terms of the operators $\psi_{\vec{k}, \sigma}^{ \pm}$, the interaction $V$ can be written

$$
\begin{aligned}
V= & \sum_{\vec{n}, \sigma, \sigma^{\prime}}\left(\frac{1}{L}\right)^{4} \sum_{\vec{k}_{1}, \vec{k}_{2}, \vec{k}_{3}, \vec{k}_{4}} \lambda \hat{v}_{n}\left(\vec{k}_{1}, \vec{k}_{2}, \vec{k}_{3}, \vec{k}_{4}\right) \delta\left(\vec{k}_{1}+\vec{k}_{2}-\vec{k}_{3}-\vec{k}_{4}+\vec{n} \frac{2 \pi}{a}\right) \\
& \times \psi_{\vec{k}_{1}, \sigma^{+}}^{+} \psi_{\vec{k}_{2}, \sigma^{\prime}}^{+} \psi_{\vec{k}_{3}, \sigma^{\prime}}^{-} \psi_{\vec{k}_{4}, \sigma}^{+},
\end{aligned}
$$

if $\delta(\vec{k})=L \delta_{\vec{k}, 0}$ and $\delta_{i, j}$ is the Kronecker delta. The presence of the periodic potential has the effect that the sum of the "incoming" and "outgoing" momenta (i.e. $\vec{k}_{1}, \vec{k}_{2}$ and $\vec{k}_{3}, \vec{k}_{4}$ respectively) is not zero but it is equal to a vector of the reciprocal lattice $2 \vec{n} \pi / a$, that is momentum is not conserved. The terms in which $\vec{n} \neq 0$ are called Umklapp terms.

If the fermions are spinless and there is no periodic potential, then one can prove rigorously, [B.G., B.G.M., B.G.P.S.] that the pair Schwinger function has an anomalous long distance behaviour. This essentially means that the occupation number at the Fermi surface is not discontinuous but is given by $n_{\vec{k}}-n_{p_{F}} \simeq|| \vec{k}\left|-p_{F}\right|^{2 \eta(\hat{\lambda})} \operatorname{sign}\left(|\vec{k}|-p_{F}\right), \eta(\lambda)=O\left(\lambda^{2}\right)$ (anomalous Fermi surface). In the present paper we derive new, corresponding, results about one dimensional interacting Fermi systems in a periodic potential, both for spinning or spinless fermions.

If the fermions are spinless and the conduction band is not filled the Fermi surface is anomalous at small enough coupling; in fact we prove:

Theorem 1.1. Given a $C^{\infty}$-smooth pair potential $\lambda v(\vec{x}-\vec{y})$ with short range $p_{0}^{-1}, a$ fermion mass $m$, a Fermi momentum $p_{F}$ and a $C^{\infty}$-smooth periodic even potential $U(\vec{x})$, and if $p_{F} \neq \frac{n \pi}{a}, n$ integer, then there exists $\varepsilon>0$ such that one can define, for $|\lambda|<\varepsilon$, functions $p_{F}(\lambda), \eta(\lambda)$ analytic in $\lambda$ and divisible by $\lambda^{2}$, such that:

1. the one dimensional spinless Fermi system with hamiltonian

$$
\sum_{i}^{n}\left(-\frac{\partial_{\vec{x}_{i}}^{2}}{2 m}+U\left(\vec{x}_{i}\right)-\mu\right)+\lambda \sum_{i<j} v\left(\vec{x}_{i}-\vec{y}_{j}\right)
$$

admits a ground state with a Euclidean pair Schwinger functions $S(k)$ verifying, for $|\vec{k}|-p_{F}^{0}$ and $k_{0}$ small, where $p_{F}^{0}=p_{F}+p_{F}(\lambda)$, the relation

$$
S(k)=S_{0}(k)\left|p_{0}^{-1} k^{\prime}\right|^{2 \eta}+A_{k}(\lambda)\left|p_{0}^{-1} k^{\prime}\right|^{-1+2 \eta},
$$

where $\left|k^{\prime}\right|=\sqrt{\left(|\vec{k}|-p_{F}^{0}\right)^{2}+v_{0}^{-2} k_{0}^{2}}, S_{0}(k)$ is the Schwinger function for the free gas with Fermi momentum $p_{F}^{0}$ and Fermi velocity $v_{0}$ and $\left|A_{k}(\lambda)\right|<C|\lambda|$, for a suitable constant $C$; 
2. $\eta(\lambda)$, the "anomalous exponent," has the expansion $\lambda^{2} \hat{v}_{0}^{2}+O\left(\lambda^{3}\right)$ with the coefficient $\hat{v}_{0}$ given by $\hat{v}_{0}=\hat{v}_{0}\left(p_{F},-p_{F},-p_{F}, p_{F}\right)$.

This result agrees with the considerations in [Sh.] based on an analogy with some solvable models, suggesting that the periodic potential in a spinless $d=1$ interacting Fermi system has "no effect" unless $\lambda=O(1)$ (i.e. the results are not qualitatively different from those of the case of translation invariant spinless fermions in the continuum). If the fermions are spinning we have that, if the interaction is small and repulsive and the conduction band is neither filled nor half filled, the Fermi surface is anomalous; more precisely we prove:

Theorem 1.2. Given a $C^{\infty}$-smooth pair potential $\lambda v(\vec{x}-\vec{y})$ with short range $p_{0}^{-1}$ and with $\hat{v}_{0}\left(-p_{F}, p_{F},-p_{F}, p_{F}\right)>0$, a fermion mass $m$, a Fermi momentum $p_{F}$ and given a $C^{\infty}$-smooth periodic even potential $U(\vec{x})$, and if $p_{F} \neq \frac{n \pi}{2 a}, n$ integer then there exists $\varepsilon>0$ such that one can define functions $p_{F}(\lambda), \eta(\lambda)$ analytic in $\lambda$ for $|\lambda-\varepsilon / 2|<\varepsilon / 2$, and divisible by $\lambda^{2}$, such that:

1. the one dimensional spinning Fermi system with hamiltonian

$$
\sum_{i}^{n}\left(-\frac{\partial_{\vec{x}_{i}}^{2}}{2 m}+U\left(\vec{x}_{i}\right)-\mu\right)+\lambda \sum_{i<j} v\left(\vec{x}_{i}-\vec{y}_{i}\right)
$$

admits a ground state with a Euclidean pair Schwinger function $S(k)$, verifying for $|\vec{k}|-p_{F}^{0}$ and $k_{0}$ small, where $p_{F}^{0}=p_{F}+p_{F}(\lambda)$, the relation

$$
S(k)=S_{0}(k)\left|p_{0}^{-1} k^{\prime}\right|^{2 \eta}+A_{k}(\lambda)\left|p_{0}^{-1} k^{\prime}\right|^{-1+2 \eta},
$$

where $\left|k^{\prime}\right|=\sqrt{\left(|\vec{k}|-p_{F}^{0}\right)^{2}+v_{0}^{-2} k_{0}^{2}}, S_{0}(k)$ is the Schwinger function for the free gas with Fermi momentum $p_{F}^{0}$ and Fermi velocity $v_{0}$ and $\left|A_{k}(\lambda)\right|<C|\lambda|$, for a suitable constant $C$;

2. $\eta(\lambda)$, the "anomalous exponent," has the expansion $\lambda^{2} \hat{v}_{0}^{2}+O\left(\lambda^{3}\right)$ with the coefficient $\hat{v}_{0}$ given by $\hat{v}_{0}=\hat{v}_{0}\left(p_{F},-p_{F},-p_{F}, p_{F}\right)$.

The above theorems show that the Fermi momentum and the Fermi velocity in the free system (i.e. $\lambda=0$ ) and in the interacting system (i.e. $\lambda \neq 0$ ) are different: one usually says that the interaction renormalizes the values of the Fermi momentum and Fermi velocity. From a physical point of view it is more natural to fix the value of these quantities in the interacting theory so that we can replace the hamiltonian Eq. (1) by an hamiltonian containing two free parameters to be tuned so that the ground state has some fixed value of the Fermi momentum and Fermi velocity (we call them simply $p_{F}$ and $v_{0}$ ). So we shall study

$$
H=T+\lambda V+\alpha T+v N,
$$

where $N=\sum_{\sigma} \int_{-L / 2}^{L / 2} d \vec{x} \psi_{\vec{x}, \sigma}^{+} \psi_{\vec{x}, \sigma}^{-}$and we prove, for instance in the spinning case, that there exists an $\varepsilon>0$ so that it is possible to fix $\alpha(\lambda), v(\lambda), \eta(\lambda)$ analytic in $|\lambda-\varepsilon / 2|<\varepsilon / 2$ and divisible by $\lambda^{2}$ such that the Euclidean pair Schwinger function verifies, for $|\vec{k}|-p_{F}, k_{0}$ small, the relation

$$
S(k)=S_{0}(k)\left|p_{0}^{-1} k^{\prime}\right|^{2 \eta}+A_{k}(\lambda)\left|p_{0}^{-1} k^{\prime}\right|^{-1+2 \eta},
$$


where $\left|k^{\prime}\right|=\sqrt{\left(|\vec{k}|-p_{F}\right)^{2}+v_{0}^{-2} k_{0}^{2}}, S_{0}(k)$ is the Schwinger function for the free gas with Fermi momentum $p_{F}$ and Fermi velocity $v_{0},\left|A_{k}(\lambda)\right|<C|\lambda|$, for a suitable constant $C$ and the $\eta(\lambda)$, "anomalous exponent," has the expansion $\lambda^{2} \hat{v}_{0}^{2}+O\left(\lambda^{3}\right)$ with the coefficient $\hat{v}_{0}$ given by $\hat{v}_{0}=\hat{v}_{0}\left(p_{F},-p_{F},-p_{F}, p_{F}\right)$. This result is of course equivalent to Theorem 1.2 , by a trivial application of a implicit function theorem. Analogous considerations can be made for the spinless case. From Eq. (10) we see that our choice of $\alpha(\lambda), v(\lambda)$ fix the value of the Fermi momentum to $p_{F}$; our choice will fix also the value of the Fermi velocity to $v_{0}$. This is not clear from Eq. (10): it is not, strictly speaking, proved here but it should be clear from the proofs that follow. We note that there are many ways different from Eq. (9) to introduce two free parameters in the hamiltonian (for instance one can have instead of $\alpha T$ a term like $\alpha \sum_{\sigma} \int_{-L / 2}^{L / 2} d \vec{x} \psi_{\vec{x}, \sigma}^{+} U(x) \psi_{\vec{x}, \sigma}^{-}$or $\left.\alpha \sum_{\sigma} \int_{-L / 2}^{L / 2} d \vec{x} \psi_{\vec{x}, \sigma}^{+}\left(\frac{\partial_{\vec{x}}^{2}}{2 m}-\mu\right) \psi_{\vec{x}, \sigma}^{-}\right)$, but it is easy to check that what follows does not depend on the particular choice Eq. (1). Finally in the cases not covered by the theorems we are not able to give rigorous results. The difficulties we met in such cases are discussed briefly in the last section.

Technical comments. The proof of the theorems is based on the renormalization group and follows the ideas in [B.G., B.G.M., B.G.P.S] for a system of spinless fermions with no periodic potential. However our proof contains new technical results necessary to treat our model. The first difficulty one meets in studying fermions in a periodic potential is that the estimates on a Bloch wave propagator are not trivial as in the case of plane waves and requires a careful analysis, for the presence of the non-analyticity points in the dispersion relation and in the Bloch waves themselves. Due to this fact and contrary to the case studied in [B.G., B.G.M., B.G.P.S] we are not able to find a definition of quasi-particles such that the free quasi-particle propagator "at scale $h$ " decays exponentially with the distance; we have only a power law decay (see below, or [B.G.P.S.], for the precise notion of quasi-particles and of scales). In [B.G.P.S.] the convergence of the anomalous Beta function, as a power series in the running coupling constants (redefined precisely below, for completeness), was proved by using in an essential way that the fixed scale quasi-particle propagators have an exponential decay; extending the proof to propagators with a weaker decay, i.e. power-law, is non-trivial and requires some new technical results (see App. 2,3).

The use, for spinning fermions, of the localization introduced in [B.G., B.G.M., B.G.P.S] leads to local terms containing irrelevant operators (this was the reason for the apparent difficulties found by [B.G.] in the spinning case). From our definition of localization we have in the spinning case six relevant running couplings when the band is not half-filled, and seven when it is half-filled; the Umklapp scattering is relevant only at half-filling. In the spinless case there are four running couplings like in the $U(\vec{x})=0$ case and Umklapp is never relevant, not even in the half-filled case (by Pauli's exclusion principle).

In the spinless case the exact solution of the Luttinger model, [M.L.], is the key to the proof that the Beta function is vanishing, the renormalization flow is bounded and that the Fermi surface is anomalous. Schwinger functions and running couplings are analytic for $|\lambda|<\varepsilon$. However there is a major difference between the spinless and spinning case, namely in the spinning case Schwinger functions are not analytic in $\lambda$ around $\lambda=0$ and this is a manifestation of the fact that the analysis of the renormalization group flow is substantially different from the spinless 
case: the beta function is not vanishing, not even to the second order. We show nevertheless that, if the conduction band is not half filled and the interaction is replusive, it is possible to control the flow, but this can be done only giving up analyticity (but retaining almost surely Borel summability). This is done proving that the Beta function is partially vanishing using the exact solution of the Mattis model [M.]. Our proof is conceptually similar to the proof in [B.G.P.S.] for the vanishing of the Beta function in the spinless case and provides the proof of some technical results stated and used in [B.G.P.S.] for this proof but not explicitly proved there.

Another noticeable difference is that our discussion is consistently performed at finite volume and temperature $\beta^{-1}$, taking care in getting bounds which are uniform in $L, \beta \rightarrow \infty$ (in [B.G.P.S.] the theory is developed directly for $\beta=\infty, L=\infty$ and the uniformity in $\beta, L$ is not really discussed). As a byproduct we extend and derive explicitly the expression of the running couplings at the cut off scale [B.G.P.S.] Eq. (7.10).

\section{Multiscale Decomposition and Effective Potential}

We consider a Grassmann algebra, whose elements $\psi_{k, \sigma}^{s}$ verify $\left\{\psi_{k, \sigma}^{s}, \psi_{k^{\prime}, \sigma^{\prime}}^{s^{\prime}}\right\}=0$. The Euclidean fields are, if $\varepsilon= \pm$,

$$
\psi_{x, \sigma}^{\varepsilon}=\frac{1}{\beta L} \sum_{k} e^{i \varepsilon k_{0} x_{0}} \phi(\vec{k}, \varepsilon \vec{x}) \psi_{k, \sigma}^{\varepsilon},
$$

where $e^{i k_{0} \beta}=-1, e^{i \vec{k} L}=1$ (see [B.G.P.S.] or [B.G.]). Although $\beta$ and $L$ are kept finite we will write $\int \frac{d k}{(2 \pi)^{2}}$ instead of $\frac{1}{\beta L} \sum_{k}$ to make the notation more clear. A "functional integration" is defined on the monomials by the Wick rule

$$
\int P(d \psi) \psi_{x_{1}, \sigma_{1}}^{+} \ldots \psi_{x_{n}, \sigma_{n}}^{+} \psi_{y_{1}, \sigma^{\prime}}^{-} \ldots \psi_{y_{n}, \sigma_{n}^{\prime}}^{-}=\sum_{\pi \in P_{n}}(-1)^{\pi} \prod_{i} g\left(x_{i}, y_{\pi(i)}\right) \delta_{\sigma_{i}, \sigma_{\pi(l)}^{\prime}},
$$

where $P_{n}$ is the set of all the permutation of $n$ elements and $(-1)^{\pi}$ is the parity of the permutation. The above integration rule is extended to a more complicated expression by linearity. We call this rule for associating numbers to grassmannian monomials, and by linear extension, to grassmannian polynomials a grassmannian integration with propagator $g(x, y)$ or a measure with propagator $g(x, y)$. For our purposes the case in which $g(x, y)$ is given by Eq. (5) will be of interest.

All the properties of the Gibbs state generated by the hamiltonian Eq. (1) at temperature $\beta^{-1}=0$ can be deduced from the functional $V_{e f f}$ defined by

$$
e^{-V_{\text {eff }}(\varphi)}=\frac{1}{\mathscr{N}} \int P(d \psi) e^{-\bar{V}(\psi+\varphi)},
$$

where $\mathscr{N}=\int P(d \psi) e^{-\bar{V}(\psi)}$ is a normalization constant (so that $V_{\text {eff }}(0)=0$ ) and $\bar{V}=\lambda V+\alpha T+v N$ with, if $x_{i}=\left(x_{0, i}, \vec{x}_{i}\right)$,

$$
\begin{gathered}
V=\sum_{\sigma, \sigma^{\prime}} \int_{\Lambda \times \Lambda} d x_{1} d x_{2} v\left(\vec{x}_{1}-\vec{x}_{2}\right) \delta\left(x_{0,1}-x_{0,2}\right) \psi_{x_{1}, \sigma}^{+} \psi_{x_{2}, \sigma^{\prime}}^{+} \psi_{x_{2}, \sigma^{\prime}}^{-} \psi_{x_{1}, \sigma}^{-}, \\
T=\sum_{\sigma} \int_{\Lambda} d x \psi_{x, \sigma}^{+}\left(-\frac{\partial_{\vec{x}}^{2}}{2 m}+U(\vec{x})-\mu\right) \psi_{x, \sigma}^{-} \quad N=\sum_{\sigma} \int_{\Lambda} d x \psi_{x, \sigma}^{+} \psi_{x, \sigma}^{-},
\end{gathered}
$$


and $\Lambda=(-\beta / 2, \beta / 2) \times(-L / 2, L / 2)$. The Schwinger functions are defined by

$$
\begin{aligned}
& S\left(x_{1}, \sigma_{1}, \ldots, x_{n}, \sigma_{n}, y_{1}, \sigma_{1}^{\prime}, \ldots, y_{n}, \sigma_{n}^{\prime}\right) \\
& \quad=\frac{1}{\mathscr{N}} \int P(d \psi) e^{-\bar{V}(\psi)} \psi_{x_{1}, \sigma_{1}}^{+} \ldots \psi_{x_{n}, \sigma_{n}}^{+} \psi_{y_{1}, \sigma_{1}^{\prime}}^{-} \ldots \psi_{y_{n}, \sigma_{n}^{\prime}}^{n} .
\end{aligned}
$$

We decompose the measure into a product of two independent measures, i.e. $P(d \psi)=P\left(d \psi^{u . v .}\right) \cdot P\left(d \psi^{i . r}\right)$. The grassmannian integral in Eq. (11) can be rewritten as

$$
\begin{aligned}
& e^{-V_{e f f}(\varphi)}=\frac{\mathscr{N}_{0}}{\mathscr{N}} \int P\left(d \psi_{i . r .}\right) e^{-V^{0}\left(\psi_{l,}+\varphi\right)}, \\
& e^{-V^{0}\left(\psi_{l . r .}+\varphi\right)}=\frac{1}{\mathscr{N}_{0}} \int P\left(d \psi_{u . v .}\right) e^{-\bar{V}\left(\psi_{i r}+\psi_{u v}+\varphi\right)},
\end{aligned}
$$

where $\psi^{u . v .}, \psi^{i . r .}, \phi$ are anticommuting grassmannian fields, $\mathscr{N}_{0}=\int P\left(d \psi_{\text {u.v. }}\right) e^{-\bar{V}\left(\psi_{\text {u.t. }}\right)}$ and $P\left(d \psi^{u . v .}\right), P\left(d \psi^{i . r}\right)$ denote respectively the grassmannian integrations with vanishing cross propagator and with propagators $g_{u . v .}, g_{\text {l.r. }}$ given by

$$
\begin{aligned}
& g_{u . v}(x, y)=\int \frac{d k}{(2 \pi)^{2}} \frac{e^{-i k_{0}\left(x_{0}-y_{0}\right)}}{-i k_{0}+E(\vec{k})} \phi(\vec{k},-\vec{x}) \phi(\vec{k}, \vec{y}) h\left(k_{0}^{2}+E\left(\vec{k}^{2}\right)\right), \\
& g_{i . r}(x, y)=\int \frac{d k}{(2 \pi)^{2}} \frac{e^{-i k_{0}\left(x_{0}-y_{0}\right)}}{-i k_{0}+E(\vec{k})} \phi(\vec{k},-\vec{x}) \phi(\vec{k}, \vec{y})\left(1-h\left(k_{0}^{2}+E(\vec{k})^{2}\right)\right),
\end{aligned}
$$

where $h(t)$ is a $C^{\infty}$ function in its argument $t$ and it is identically 1 if $t>\mu^{2}$, hence $h\left(k_{0}^{2}+E(\vec{k})^{2}\right)$ is identically 1 if $\vec{k}$ is above the first band $(|\vec{k}|>\pi / a)$ and it is also identically 1 for $k_{0}, \vec{k}$ near $(0,0)$ : this property shows that the integral in $g_{u . v}(x, y)$ involves only $\left(k_{0}, \vec{k}\right)$ 's which are "far" from the Fermi surface $k_{0}=0, E(\vec{k})=0$, thus justifing the u.v. name.

It is possible to check that (see App. 1)

$$
\begin{aligned}
g_{u . v .}(x, y) & =G(x-y)+R(x, y) \\
G(x) & =H(\vec{x}-\vec{y}) H\left(x_{0}-y_{0}\right) \theta\left(x_{0}-y_{0}\right) e^{\left(x_{0}-y_{0}\right) \mu}\left(\frac{m}{2 \pi\left(x_{0}-y_{0}\right)}\right)^{1 / 2} e^{-\frac{m(\vec{x}-\vec{y})^{2}}{2\left(x_{0}-y_{0}\right)}},
\end{aligned}
$$

where $R(x, y) \leqq \frac{C_{N}}{1+\left(p_{0}|x-y|\right)^{N}},|x|^{2}=x_{0}^{2} v_{0}^{2}+\vec{x}^{2}$ for all $N$, and $H(t)$ is a smooth function of compact support such that $H(t)=1$, if $|t| \leqq 1$ and $H(t)=0$ if $|t| \geqq \gamma \geqq 1$.

The Eq. (16) shows that $g_{u . v}$. has the same properties of the ultraviolet propagator with $U(\vec{x})=0$, so that we can repeat the proofs leading to the theory of the ultraviolet problem for the $U(\vec{x})=0$ case in [B.G.P.S.], (it is trivial to include the presence of the spin in developing the proof). In [B.G.P.S.] as well as in our case the following statement is rather easy and the difficult part does not concern the ultraviolet problem but only the infrared one:

Theorem 2.1. There exists $\varepsilon>0$ such that $V^{0}$, see $E q(14)$, can be written for $|z| \leqq \varepsilon, z=(\lambda, \alpha, v)$ and if $\psi \equiv \psi^{i . r}$, in the following way as a sum of a term 
linear in the couplings:

$$
\begin{aligned}
V^{0}(\psi)= & \sum_{\sigma, \sigma^{\prime}} \lambda \int_{\Lambda} d x d y v(x-y) \psi_{x, \sigma}^{+} \psi_{y, \sigma^{\prime}}^{+} \psi_{y, \sigma^{\prime}}^{-} \psi_{x, \sigma}^{-}+2 \lambda \int d x d y v(x-y) R(x, y) \psi_{x, \sigma}^{+} \psi_{y, \sigma}^{-} \\
& +\sum_{\sigma} \int_{\Lambda} d x(v-2 \lambda K(x)) \psi_{x, \sigma}^{+} \psi_{x, \sigma}^{-}+\alpha \sum_{\sigma} \int_{\Lambda} d x \psi_{x, \sigma}^{+}\left(-\frac{\Delta}{2 m}+U(x)-\mu\right) \psi_{x, \sigma}^{-}
\end{aligned}
$$

with $K(x)=\int_{\Lambda} v(\vec{x}-\vec{y}) R(y, y) d y$ and of a remainder of order at least 2 in the couplings $\lambda, \alpha, v$,

$$
\begin{aligned}
& \sum_{\sigma} \int_{\Lambda} d x \psi_{x, \sigma}^{+}\left(-\frac{\Delta}{2 m}+U(x)-\mu\right) \psi_{y, \sigma}^{-} W_{2}(x, y ; z) \\
& \quad+\sum_{\sigma_{1}, \ldots, \sigma_{n}} \sum_{\substack{n, n_{1}, n_{2} \\
n_{1}+n_{2}=n}} \int d x_{1} \ldots d x_{2 n} \psi_{x_{1}}^{+} \ldots \psi_{x_{n_{1}}}^{+} \psi_{x_{n_{1}+1}}^{-} \psi_{x_{2 n-n_{2}}}^{-} \\
& \quad \times\left(-\frac{\Delta}{2 m}+U\left(x_{2 n-n_{2}}\right)-\mu\right) \psi_{x_{2 n-n_{2}+1}}^{-} \ldots\left(-\frac{\Delta}{2 m}+U\left(x_{2 n}\right)-\mu\right) \\
& \quad \times \psi_{x_{2 n}}^{-} W_{n_{1}, n_{2}}\left(z, x_{1}, \ldots, x_{2 n}\right),
\end{aligned}
$$

where the kernels $W_{n_{1}, n_{2}}$ are products of suitable delta functions times bounded functions analytic in $z$ if $|z| \leqq \varepsilon$ and, if $d\left(x_{1} \ldots, x_{n}\right)$ is the length of the shortest tree connecting the points ("tree distance" or "graph distance"), the following bounds hold:

$$
\int d x_{1} \ldots d x_{2 n}\left|W_{n_{1}, n_{2}}\left(z, x_{1}, \ldots\right)\right|\left(1+d\left(x_{1}, \ldots, x_{n}\right)\right)^{N}<c(N) \Lambda|z|^{\max (2, n-1)}
$$

while $W_{2}$ satisfies the weaker bound

$$
\left|\int d x d y \phi\left(\vec{k}_{1}, \vec{x}\right) \phi\left(-\vec{k}_{2}, \vec{y}\right) e^{-l k_{0}\left(x_{0}-y_{0}\right)} W_{2}(x, y ; z)\right| \leqq D|z|^{2}
$$

for $\left|\vec{k}_{1}\right|,\left|\vec{k}_{2}\right| \leqq \pi / a$ and $|z| \leqq \varepsilon$.

A more interesting and difficult problem is the analysis of the "infrared" integration Eq. (13): We decompose the grassmannian integration $P(d \psi)$ into a product of independent grassmannian integrations, that is $P\left(d \psi_{i . r .}\right)=\prod_{h=-\infty}^{0} P\left(d \psi^{h}\right)$. This can be done by setting $g_{l . r .}(k)=\sum_{h=-\infty}^{0} g^{h}(k)$ and by writing $\psi_{i . r .}=\sum_{h} \psi^{(h)}$, with $\psi^{h}$ being a family of grassmannian fields with vanishing "cross propagator" (i.e. independent) and with propagator $\int \psi_{k_{1}, \sigma_{1}}^{h} \psi_{k_{2}, \sigma_{2}}^{h} P\left(d \psi^{h}\right)=\delta_{\sigma_{1}, \sigma_{2}} \delta\left(k_{1}-k_{2}\right) g^{h}\left(k_{1}\right)$ :

$$
g^{h}(k)=\frac{f\left(\gamma^{-2 h+2}\left(k_{0}^{2}+E(\vec{k})^{2}\right)\right)}{-i k_{0}+E_{0}(\vec{k})},
$$

where $f(x)=h(x)\left(1-h\left(\frac{x}{\gamma^{2}}\right)\right)$ is a $C^{\infty}$ function with compact support and $\gamma>1$. However such decomposition is not suitable for a renormalization group analysis, because $g^{h}(k)$ have no good scaling properties (as the system has two intrinsic scale lengths, i.e. $a$ and $p_{F}$ ). In order to overcome this difficulty we introduce new grassmannian fields $\psi_{k, \vec{\omega}, \sigma}^{h}$, called quasi-particles field operators, with propagators 
Fermi Surface for a $d=1$ Interacting Fermions in Periodic Potential

$g_{\vec{\omega}}^{(h)}(k)$ and vanishing cross propagators, such that

$$
\psi_{k, \sigma}^{h}=\sum_{\vec{\omega}= \pm 1} \psi_{k, \vec{\omega}, \sigma}^{h}, \quad \psi_{k, \sigma}^{h}=\sum_{\vec{\omega}= \pm 1} \psi_{k, \vec{\omega}, \sigma}^{h}, g^{h}(k)=\sum_{\vec{\omega}= \pm 1} g_{\vec{\omega}}^{h}(k) .
$$

Although there are infinitely many ways to represent $g^{h}(k)$ in this form, there is at least one such that $g_{\vec{\omega}}^{h}(k)$ has good scaling property. Define

$$
g^{h}(k)=\sum_{\vec{\omega}= \pm 1} \theta(\vec{\omega} \vec{k}) \frac{f\left(\gamma^{-2 h+2}\left(k_{0}^{2}+E(\vec{k})^{2}\right)\right)}{-i k_{0}+E(\vec{k})},
$$

where $\theta$ is the step function. If $k=k^{\prime}+\left(0, \vec{\omega} p_{F}\right)$, i.e. $\vec{k}^{\prime}$ is the momentum measured from the Fermi surface and is restricted to values of the form $\frac{2 \pi}{L}(n+1 / 2)$, where $n$ is an integer, it is possible to prove that:

Lemma 2.1. If $v_{0}$ is different from zero then the quasi-particle propagator can be written as

$$
\gamma^{-h} g_{\vec{\omega}}^{h}\left(\gamma^{-h} k^{\prime}\right)=\gamma^{-h} \bar{g}_{\vec{\omega}}^{h}\left(\gamma^{-h} k^{\prime}\right)+C_{h}\left(\gamma^{-h} k^{\prime}\right),
$$

where

$$
\bar{g}_{\vec{\omega}}^{h}\left(\gamma^{-h} k^{\prime}\right)=\frac{f\left(\gamma^{2}\left(\left[\gamma^{-h} k_{0}\right]^{2}+\left(\left[\gamma^{-h} \overrightarrow{k^{\prime}}\right] v_{0}\right)^{2}\right)\right.}{-i\left[\gamma^{-h} v_{0} k_{0}\right]+\vec{\omega}\left[\gamma^{-h}{\overrightarrow{k^{\prime}}}^{\prime}\right.} v_{0},
$$

and $C_{h}(t)$ is $C^{\infty}$ with support contained in $(-\pi / a, \pi / a)$ and such that $\left|C_{h}(t)\right| \leqq M$, where $M$ does not depend on $h, T, L$.

Note that $v_{0}=0$ only if $p_{F}=0, \pi / a$, as we have supposed that $\left|p_{F}\right| \leqq \pi / a$.

We define the "position space" quasi-particle fields as

$$
\begin{aligned}
\hat{\psi}_{x, \sigma}^{h, \pm} & =\int \frac{d k}{(2 \pi)^{2}} e^{ \pm i\left(k_{0} t+\vec{k} \vec{x}\right)} \psi_{k, \sigma}^{h} \quad \hat{\psi}_{x, \sigma}^{h, \pm}=\sum_{\vec{\omega}= \pm 1} e^{ \pm l \vec{\omega} p_{F} \vec{x}} \hat{\psi}_{x, \vec{\omega}, \sigma}^{h, \pm}, \\
\hat{\psi}_{x, \vec{\omega}, \sigma}^{h, \pm} & =\int \frac{d k^{\prime}}{(2 \pi)^{2}} e^{ \pm i\left(k_{0} t+\vec{k} \cdot \vec{x}\right)} \psi_{k^{\prime}+\vec{\omega} p_{F}, \vec{\omega}, \sigma}^{h} \quad \hat{g}_{\vec{\omega}}^{h}(x)=\int \frac{d k^{\prime}}{(2 \pi)^{2}} e^{-l\left(k_{0} t+\vec{k} \cdot \vec{x}\right)} g_{\vec{\omega}}^{h}\left(k^{\prime}\right) .
\end{aligned}
$$

Note that, at variance with the work in the translation invariant case, [B.G.], the fields $\sum_{h, \vec{\omega}} e^{ \pm i \omega \vec{\omega} p_{F} \vec{x}} \hat{\psi}_{x, \sigma, \vec{\omega}}^{h, \pm}$ cannot be identified with the fields $\psi_{x, \sigma}^{l . r}$ above: this is an important difference with respect to [B.G.]. The relation between $\hat{\psi}_{x, \sigma, \vec{\omega}}^{h, \pm}$ and $\psi_{x, \sigma}^{i . . \pm}$ is more complicated as it is given by

$$
\psi_{x, \sigma}^{l . r . \pm}=\int d k \phi(\vec{k}, \pm \vec{x}) e^{ \pm \imath k_{0} x_{0}} \int d x^{\prime} e^{\mp i k x^{\prime}} \sum_{h, \vec{\omega}} e^{ \pm i \vec{\omega} p_{F} \vec{x}^{\prime}} \hat{\psi}_{x^{\prime}, \vec{\omega}, \sigma}^{h, \pm}
$$

The natural definition, if we wanted to operate in analogy with [B.G.], would be introducing the field

$$
\psi_{x, \vec{\omega}, \sigma}^{h, \pm}=\int \frac{d k^{\prime}}{(2 \pi)^{2}} e^{\mp l k_{0} x_{0}} \frac{\phi\left(\overrightarrow{k^{\prime}}+\vec{\omega} p_{F}, \pm x\right)}{\phi\left(\vec{\omega} p_{F}, \pm \vec{x}\right)} \psi_{k^{\prime}+\vec{\omega} p_{F}, \vec{\omega}, \sigma}^{h, \pm}
$$

with propagator $g_{\vec{\omega}}^{h}(x, y)$ and to set $\psi_{x, \sigma}^{i, r, \pm}=\sum_{\vec{\omega}, h} \phi\left(\vec{\omega} p_{F}, \pm \vec{x}\right) \psi_{x, \vec{\omega}, \sigma}^{h, \pm}$. Such definition would be "the same" as in [B.G.], with of course plane waves replaced by Block waves, but the definition of "localization" would become very cumbersome, so we prefer not to use these fields. 
It is not difficult to check that:

Lemma 2.2. For $N>1$ we have:

$$
\begin{aligned}
& \hat{g}_{\vec{\omega}}^{h}(x)<\gamma^{h} \frac{C_{N}\left(a, p_{F}\right)}{\left.1+p_{0}^{-N}\left[\gamma^{2 h}(\vec{x})_{\pi}^{2}+v_{0}^{2} \gamma^{2 h}\left(x_{0}\right)_{\pi}^{2}\right)\right]^{N / 2}}, \\
& \hat{C}_{h}(x)<\gamma^{2 h} \frac{C_{N}\left(a, p_{F}\right)}{\left.1+p_{0}^{-N}\left[\gamma^{2 h}(\vec{x})_{\pi}^{2}+\gamma^{2 h} v_{0}^{2}\left(x_{0}\right)_{\pi}^{2}\right)\right]^{N / 2}},
\end{aligned}
$$

where $\hat{C}_{h}(x)$ is the Fourier transform of $C_{h}\left(\gamma^{-h} \vec{k}\right),(\vec{x})_{\pi}=\frac{\sin \vec{x} \pi / L}{\pi / L}$ and $\left(x_{0}\right)_{\pi}=$ $\frac{\sin \pi / \beta}{\pi / \beta}$. Moreover

$\hat{g}_{\vec{\omega}}^{h}(x) \leqq \gamma^{h} \frac{C_{N}\left(a, p_{F}\right)}{1+\gamma^{h N}\left|p_{0} x\right|^{N}} \quad \hat{C}_{h}(x) \leqq \gamma^{2 h} \frac{C_{N}\left(a, p_{F}\right)}{1+\gamma^{h N}\left|p_{0} x\right|^{N}}$ for $|\vec{x}| \leqq L / 2,\left|x_{o}\right| \leqq \beta / 2$

with $|x|^{2}=v_{0}^{2} x_{0}^{2}+\vec{x}^{2}$.

One could hope that, by using an analytic (rather than $C^{\infty}$ ) cut off function $h$ to realise the decomposition Eqs. (15),(19), and by making a shift in the integral of the infrared propagator $g_{\vec{\omega}}^{h}(x, y)$ or $\hat{g}_{\vec{\omega}}^{h}(x)$ to a complex line with imaginary part $\gamma^{h} \bar{h}, \gamma^{h} \bar{h} \neq h_{n}$, following a path similar to that of Appendix 1, one would obtain that $\hat{g}_{\vec{\omega}}^{h}(x)$ decays exponentially for large distance. However this does not happen: essentially because one cannot use in this case the symmetry properties used in Appendix 1 and because of the accumulation of the non-analyticity points on the real $\vec{k}$ line. We strongly suspect that there is no way to realize a multiscale decomposition for our problem such that the quasi-particles have propagator with exponential decay in the $x$-variables. In any event we were not able to find it: so that we preferred a compact support cut-off. In this way the analysis of the perturbative expansion is clearer, as the distinction between ultraviolet and infrared term is sharper.

\section{The Effective Potential in the Infrared Region}

In this section we set $v_{0}=1$ for simplicity and we begin the analysis of the infrared problem, which consists in the study of the possibility to give a rigorous meaning to $V_{\text {eff }}(\phi)$ defined by the functional integration Eq. (13). We start by studying the functional integral $\int P\left(d \psi_{i . r}\right) e^{-V^{0}\left(\psi_{i . r}\right)}$, which is the normalization constant in Eq. (9). We can represent $V^{0}(\psi)$, see Eq. (17), (18), in terms of quasi-particles fields $\psi_{\vec{k}, \vec{\omega}, \sigma}^{\varepsilon}$, where $\varepsilon= \pm 1$, so that $V^{0}(\psi)$ is given by a sum of terms like

$$
\begin{gathered}
V_{m}^{0}(\psi)=\int \frac{d k_{1}^{\prime}}{(2 \pi)^{2}} \ldots \frac{d k_{n}^{\prime}}{(2 \pi)^{2}} f^{m}\left(k_{1}^{\prime}, \ldots, k_{m}^{\prime}, ; \underline{\vec{\omega}}\right) \delta\left(\sum_{l=1}^{m}\left(k_{i}^{\prime}+\vec{\omega}_{i} p_{F}\right) \varepsilon_{i}+\frac{2 n \pi}{a}\right) \\
\prod_{i=1}^{m} \psi_{k_{l}^{\prime}+\vec{\omega}_{i} p_{F}, \vec{\omega}_{i}, \sigma_{i}}^{\varepsilon_{\varepsilon_{i}}} .
\end{gathered}
$$

We can isolate the relevant part of $V^{0}(\psi)$ by introducing a localization operator $\mathscr{L}$ on the Fermi surface acting on $V^{0}(\psi)$ as follows; $\mathscr{L} V_{m}^{0}(\psi)=0$ for $m>4$, while (see discussion after Eq. (30), (31) for a motivation of the localization name given to $\mathscr{L}$ ) a "natural" definition for $\mathscr{L}$ if $m=2,4$ should be to 
computing $f^{m}\left(k_{1}^{\prime}, \ldots, k_{m}^{\prime}, ; \underline{\vec{\omega}}\right)$ and (if $\left.m=2\right)$ its derivative at the Fermi surface, i.e. for $k_{1}^{\prime}=, \ldots,=k_{m}^{\prime}=0$. However $k^{\prime}$ cannot assume the value 0 as $k^{\prime}=k-\left(0, \vec{\omega} p_{F}\right)$ has the form $k^{\prime}=\left(2 \pi / L\left(n_{1}+1 / 2\right), 2 n_{2} \pi / \beta+1 / 2\right)$, with $n_{1}, n_{2}$ integer, for the antiperiodic boundary temporal conditions and the definition of $p_{F}=2 \pi / L\left(n_{F}+1 / 2\right)$, and this takes to the complicated formulae below:

$$
\begin{array}{rl}
\mathscr{L} \int \prod_{l=1}^{4} & d k_{i}^{\prime} f^{4}\left(k_{1}^{\prime}, k_{2}^{\prime}, k_{3}^{\prime}, k_{4}^{\prime} ; \underline{\vec{\omega}}\right) \delta\left(k_{1}^{\prime}+k_{2}^{\prime}-k_{3}^{\prime}-k_{4}^{\prime}\right. \\
& \left.+\left(\vec{\omega}_{1}+\vec{\omega}_{2}-\vec{\omega}_{3}-\vec{\omega}_{4}\right) p_{F}+\frac{2 n \pi}{a}\right) \\
& \psi_{k_{1}^{\prime}+\vec{\omega}_{1} p_{F}, \vec{\omega}_{1}, \sigma} \psi_{k_{2}^{\prime}+\vec{\omega}_{2} p_{F}, \vec{\omega}_{2}, \sigma^{\prime}}^{+} \psi_{k_{3}^{\prime}+\vec{\omega}_{3} p_{F}, \vec{\omega}_{3}, \sigma^{\prime}} \psi_{k_{4}^{\prime}+\vec{\omega}_{4} p_{F}, \vec{\omega}_{4}, \sigma} \\
= & \delta_{\left(\vec{\omega}_{1}+\vec{\omega}_{2}-\vec{\omega}_{3}-\vec{\omega}_{4}\right) p_{F}+n \frac{2 x}{a}, 0} f^{4, L}(\underline{\vec{\omega}}) \int \prod_{i-1}^{4} d k_{i}^{\prime} \delta\left(k_{1}^{\prime}+k_{2}^{\prime}-k_{3}^{\prime}-k_{4}^{\prime}\right) \\
& \times \psi_{k_{1}^{\prime}+\vec{\omega}_{1} p_{F}, \vec{\omega}_{1}, \sigma} \psi_{k_{2}^{\prime}+\vec{\omega}_{2} p_{F}, \vec{\omega}_{2}, \sigma^{\prime}} \psi_{k_{3}^{\prime}+\vec{\omega}_{3} p_{F}, \vec{\omega}_{3}, \sigma^{\prime}} \psi_{k_{4}^{\prime}+\vec{\omega}_{4} p_{F}, \vec{\omega}_{4}, \sigma},
\end{array}
$$

where $\delta_{a, b}$ is the Kronecher delta equal to 1 if $a=b$ and zero otherwise;

$$
\begin{aligned}
\mathscr{L} \int d & k_{1}^{\prime} d k_{2}^{\prime} \delta\left(k_{1}^{\prime}-k_{2}^{\prime}+\left(\vec{\omega}_{1}-\vec{\omega}_{2}\right) p_{F}+2 n \pi / a\right) f^{2}\left(k_{1}^{\prime}, k_{2}^{\prime} ; \underline{\vec{\omega}}\right) \\
& \times \psi_{k_{1}^{\prime}+\vec{\omega}_{1} p_{F}, \vec{\omega}_{1}, \sigma} \psi_{k_{2}^{\prime}+\vec{\omega}_{2} p_{F}, \vec{\omega}_{2}, \sigma}^{-}= \\
& \delta_{\left(\vec{\omega}_{1}-\vec{\omega}_{2}\right) p_{F}+2 n \pi / a, 0} \int d k_{1}^{\prime} d k_{2}^{\prime} \delta\left(k_{1}^{\prime}-k_{2}^{\prime}\right)\left[f^{2, L_{a}}(\underline{\vec{\omega}})+E\left(\vec{K}_{1}^{\prime}+\vec{\omega}_{1} p_{F}\right)\right. \\
& \left.\times \vec{\omega}_{1} f^{2, L_{b}}(\underline{\vec{\omega}})+k_{1}^{0} f^{2, L_{c}}(\underline{\overrightarrow{\vec{\omega}}})\right] \psi_{k_{1}^{\prime}+\vec{\omega}_{1} p_{F}, \vec{\omega}_{1}, \sigma} \psi_{k_{2}^{\prime}+\vec{\omega}_{2} p_{F}, \vec{\omega}_{2}, \sigma},
\end{aligned}
$$

where if $\tilde{\partial}_{\vec{k}} f(\vec{k})$, the discrete derivative, is defined for instance as $\frac{f(\vec{k}+2 \pi / L)-f(\vec{k})}{2 \pi / L}$, and an analogous definition is set for $k_{0}$, and:

$$
\begin{aligned}
f_{h_{v_{0}}}^{4, L}(\underline{\vec{\omega}})= & \frac{1}{4} \sum_{l, j=1}^{2} f_{h_{v_{0}}}^{4}\left[\left((-1)^{i} \frac{\pi}{\beta},(-1)^{j} \frac{\pi}{L}\right),\left((-1)^{l} \frac{\pi}{\beta},(-1)^{j} \frac{\pi}{L}\right),\right. \\
& \left.\left((-1)^{i} \frac{\pi}{\beta},(-1)^{j} \frac{\pi}{L}\right),\left((-1)^{i} \frac{\pi}{\beta},(-1)^{j} \frac{\pi}{L}\right) ; \underline{\vec{\omega}}\right], \\
f_{h_{v_{0}}}^{2, L_{a}}(\underline{\vec{\omega}})= & \frac{1}{4} \sum_{l, j=1}^{2} f_{h_{v_{0}}}^{2}\left((-1)^{l} \frac{\pi}{\beta},(-1)^{j} \frac{\pi}{L} ; \underline{\vec{\omega}}\right), \\
f_{h_{v_{0}}}^{2, L_{b}}(\underline{\underline{\vec{\omega}}})= & \frac{1}{2} \sum_{i=1}^{2} \tilde{\partial}_{\vec{k}_{1}^{\prime}} f_{h_{l}}^{2}\left((-1)^{l} \frac{\pi}{\beta},-\frac{\pi}{L} ; \underline{\vec{\omega}}\right), \\
f_{h_{v_{0}}}^{2, L_{c}}(\underline{\underline{\vec{\omega}}})= & \frac{1}{2} \sum_{i=1}^{2} \tilde{\partial}_{k_{1}^{0}} f_{h_{v_{0}}}^{2}\left(-\frac{\pi}{\beta},(-1)^{l} \frac{\pi}{L} ; \underline{\vec{\omega}}\right) .
\end{aligned}
$$

In Eq. (22) the Kroneker $\delta$ can be satisfied only by $n=0$ and $\vec{\omega}_{1}=\vec{\omega}_{4}=-\vec{\omega}_{2}=$ $-\vec{\omega}_{3}, \vec{\omega}_{1}=\vec{\omega}_{3}=-\vec{\omega}_{2}=-\vec{\omega}_{4}$ or $\vec{\omega}_{1}=\vec{\omega}_{2}=\vec{\omega}_{3}=\vec{\omega}_{4}$ unless $p_{F}=\pi / 2 a$, i.e. the conduction band is half filled, in which case the Kroneker $\delta$ can also be satisfied also by $n=1$ and $\vec{\omega}_{1}=\vec{\omega}_{2}=-\vec{\omega}_{3}=-\vec{\omega}_{4}$, i.e. umklapp is relevant only if the conduction band is half filled. In Eq. (23) we must have $n=0$ and $\vec{\omega}_{1}=\vec{\omega}_{2}$. 
The relevant part of $V^{0}(\psi)$ in the spinning case is then

$$
\begin{aligned}
& \mathscr{L} V^{0}=v_{0} F_{v}+\alpha_{0} F_{\alpha}+\zeta_{0} F_{\zeta}+g_{1,0} F_{1}+g_{2,0} F_{2}+g_{3,0} \delta_{p_{F}, \pi / 2 a} F_{3}+g_{4,0} F_{4}, \\
& F_{v}=\sum_{\vec{\omega}, \sigma} \int d k_{1} d k_{2} \psi_{k_{1}^{\prime}+\vec{\omega} p_{F}, \vec{\omega}, \sigma} \psi_{k_{2}^{\prime}+\vec{\omega} p_{F}, \vec{\omega}, \sigma}^{-} \delta\left(k_{1}^{\prime}-k_{2}^{\prime}\right), \\
& F_{\alpha}=\sum_{\vec{\omega}, \sigma} \int d k_{1} d k_{2} E\left(\vec{k}_{1}^{\prime}+\vec{\omega} p_{F}\right) \psi_{k_{1}^{\prime}+\vec{\omega} p_{F}, \vec{\omega}, \sigma}^{+} \psi_{k_{2}^{\prime}+\vec{\omega} p_{F}, \vec{\omega}, \sigma}^{-} \delta\left(k_{1}^{\prime}-k_{2}^{\prime}\right) \\
& F_{\zeta}=\sum_{\vec{\omega}, \sigma} \int d k_{1} d k_{2}-i k_{1}^{0} \psi_{k_{1}^{\prime}+\vec{\omega} p_{F}, \vec{\omega}, \sigma} \psi_{k_{2}^{\prime}+\vec{\omega} p_{F}, \vec{\omega}, \sigma} \delta\left(k_{1}^{\prime}-k_{2}^{\prime}\right) \\
& F_{1}=\sum_{\vec{\omega}, \sigma, \sigma^{\prime}} \int \prod_{i=1}^{4} d k_{i} \psi_{k_{1}^{\prime}+\vec{\omega} p_{F}, \vec{\omega}, \sigma}^{+} \psi_{k_{2}^{\prime}-\vec{\omega} p_{F},-\vec{\omega}, \sigma^{\prime}}^{+} \psi_{k_{3}^{\prime}+\vec{\omega} p_{F}, \vec{\omega}, \sigma^{\prime}}^{-} \psi_{k_{4}^{\prime}-\vec{\omega} p_{F},-\vec{\omega}, \sigma}^{-} \delta\left(\sum_{l} \varepsilon_{l} k_{i}^{\prime}\right), \\
& F_{2}=\sum_{\vec{\omega}, \sigma, \sigma^{\prime}} \int \prod_{i=1}^{4} d k_{i}^{\prime} \psi_{k_{1}^{\prime}+\vec{\omega} p_{F}, \vec{\omega}, \sigma}^{+} \psi_{k_{2}^{\prime}-\vec{\omega} p_{F},-\vec{\omega}, \sigma^{\prime}}^{+} \psi_{k_{3}^{\prime}-\vec{\omega} p_{F},-\vec{\omega}, \sigma^{\prime}}^{-} \psi_{k_{4}^{\prime}+\vec{\omega} p_{F}, \vec{\omega}, \sigma}^{-} \delta\left(\sum_{i} \varepsilon_{i} k_{l}^{\prime}\right), \\
& F_{3}=\delta_{p_{F}, \pi / 2 a} \sum_{\vec{\omega}, \sigma, \sigma^{\prime}} \int \prod_{l=1}^{4} d k_{i}^{\prime} \psi_{k_{1}^{\prime}+\vec{\omega} p_{F}, \vec{\omega}, \sigma}^{+} \psi_{k_{2}^{\prime}+\vec{\omega} p_{F}, \vec{\omega}, \sigma^{\prime}}^{+} \psi_{k_{3}^{\prime}-\vec{\omega} p_{F},-\vec{\omega}, \sigma^{\prime}}^{-} \psi_{k_{4}^{\prime}-\vec{\omega} p_{F},-\vec{\omega}, \sigma}^{-} \\
& \delta\left(\sum_{l} \varepsilon_{i} k_{i}^{\prime}\right) \\
& F_{4}=\sum_{\vec{\omega}, \sigma, \sigma^{\prime}} \int \prod_{i=1}^{4} d k_{l}^{\prime} \psi_{k_{1}^{\prime}+\vec{\omega} p_{F}, \vec{\omega}, \sigma}^{+} \psi_{k_{2}^{\prime}+\vec{\omega} p_{F}, \vec{\omega},-\sigma}^{+} \psi_{k_{3}^{\prime}+\vec{\omega} p_{F}, \vec{\omega},-\sigma}^{-} \psi_{k_{4}^{\prime}+\vec{\omega} p_{F}, \vec{\omega}, \sigma}^{-} \delta\left(\sum_{l} \varepsilon_{l} k_{l}^{\prime}\right),
\end{aligned}
$$

where $\varepsilon_{1}=\varepsilon_{2}=-\varepsilon_{3}=-\varepsilon_{4}=1$. We note that the relevant part of $V^{0}(\psi)$ is similar to the phenomenological hamiltonian introduced in [S.] via heuristic considerations. Note, moreover, that there are no terms renormalizing the periodic potential in the relevant part of $V^{0}(\psi)$. In the spinless case $\sigma=0$ and $\mathscr{L} V_{h}=v_{0} F_{v}+\alpha_{0} F_{\alpha}+\zeta_{0} F_{\zeta}+$ $\lambda_{0} F$, where

$$
F=\sum_{\vec{\omega}} \int \prod_{i=1}^{4} d k_{i}^{\prime} \psi_{k_{1}^{\prime}+\vec{\omega} p_{F}, \vec{\omega}}^{+} \psi_{k_{2}^{\prime}-\vec{\omega} p_{F},-\vec{\omega}}^{+} \psi_{k_{3}^{\prime}-\vec{\omega} p_{F},-\vec{\omega}} \psi_{k_{4}^{\prime}+\vec{\omega} p_{F}, \vec{\omega}} \delta\left(\sum_{i} \varepsilon_{l} k_{l}^{\prime}\right) .
$$

Therefore, in the spinless case, $g_{2, h}=g_{1, h}=\lambda_{h}$ and $F_{3}=F_{4}=0$ (because of the anticommuting property of the grassmannian variables).

The most natural definition for the effective potential on scale $\gamma^{-k}, k<0$ for the infrared problem (but not the correct one, as it will appear clear in the following) would be:

$$
\left.e^{-V^{h}\left(\psi^{\leqq h}\right)}=\frac{\mathscr{N}^{0}}{\mathscr{N}} \int P\left(d \psi^{h+1}\right) \ldots P\left(d \psi^{0}\right) e^{-V^{0}(\psi \leqq 0}\right)
$$

where $\psi^{\leqq k}=\sum_{h=-\infty}^{k} \psi^{h}$. The inductive evaluation of Eq. (25) is made by writing at each step $V^{h}\left(\psi^{h}\right)=\mathscr{L} V^{h}\left(\psi^{h}\right)+\mathscr{R} V^{h}\left(\psi^{h}\right)$, where $\mathscr{L} V^{h}$ is given by an equation like Eq. (24) with $\left(v_{0}, \alpha_{0}, \zeta_{0}, g_{1,0}, g_{2,0}, g_{3,0}, g_{4,0}\right)$ replaced by $\left(\gamma^{h} v_{h}, \alpha_{h}, \zeta_{h}, g_{1, h}, g_{2, h}\right.$, $\left.g_{3, h}, g_{4, h}\right) \equiv \vec{v}_{h}$ plus an additive constant $t_{h}|\Lambda|,|\Lambda|=L \beta$, i.e. the vacuum contribution. The quantities $\gamma^{h} v_{h}, \alpha_{h}, \zeta_{h}$ are $\vec{\omega}$ independent (by the "rotation invariance" of 


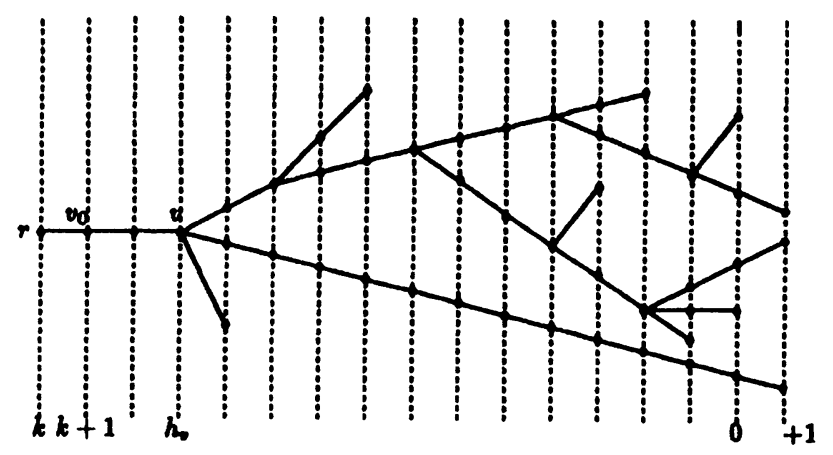

Fig. 1.

the theory, i.e. by the invariance under the transformation $\vec{x} \rightarrow-\vec{x}$ ). An essential role in this analysis will be played by the tree expansion.

We call $\tau_{n}$ the set of all the labeled trees with $n$ end points $\tau \in \tau_{n}$ that can be constructed as follows (see also the picture). Draw on the $(x, y)$ plane vertical lines at $x=k, k+1, \ldots, 0,1$. Let $\tau$ (the root) be a point on the line $x=k$. Starting from $\tau$ draw an horizontal line leading to a point $v_{0}$ on the line $x=k_{v_{0}}=k+1$. Choose $s_{v_{o}} \geqq 0$ and draw $s_{v_{0}}$ lines starting from $v_{0}$ leading to $s_{v}$ points $v_{1}, \ldots v_{s_{v}}$ on the line $x=k_{v_{l}}=k_{v}+1$, i.e. the lines cannot go back. Do the same thing starting with the points $v_{i}$ and go on recursively. A point $v$ is called an end point if $s_{v}=0$, i.e. if there is no line starting from this point. Moreover a point $v$ is a trivial vertex if $s_{v}=1$ and a non-trivial vertex if $s_{v} \geqq 2$. Finally if $h_{v}=1$, then $v$ is necessarily an end point. Clearly this process ends when all the reached points are end points. A cluster $v$ with frequency $h_{v}$ is the set of the end-points reachable from the vertex $v$ with frequency $h_{v}$; and the tree provides an organisation of the endpoints into a hierarchy of clusters. Each non-trivial or trivial vertex bears a label $\mathscr{R}$ except $v_{0}$ (see the picture) on which can bear either a label $\mathscr{R}$ or a lable $\mathscr{L}$. To each tree we associate a term $V^{(k)}\left(\tau, \psi^{(\leqq k)}\right)$ defined recursively as follows. If $\tau$ has only one end-point with frequency $k+1$ then $V^{(k)}\left(\tau, \psi^{(\leqq k)}\right)$ is equal to one of the terms of Eq. (24) with $\vec{v}_{k}$ instead of $\vec{v}_{0}$ or, only if $k=0$, one of the monomial in $\mathscr{R} V^{0}$. We attach a label to each endpoint of the tree to distinguish among these possibilities. Otherwise

$$
\left.V^{(k)}\left(\tau, \psi^{(\leqq k)}\right)=\mathcal{O} \frac{1}{s_{v} !} \mathscr{E}_{k+1}^{T}\left[V^{(k+1)}\left(\tau^{1}, \psi^{(\leqq k+1)}\right), \ldots\right)\right]
$$

where $\mathcal{O}$ is $\mathscr{L}$ or $\mathscr{R}$ if the vertex $v$ bears an $\mathscr{L}$ or $\mathscr{R}$ label, $n \geqq 2, \tau^{1} \ldots \tau^{s_{v}}$ are the subtrees starting from $v$ and the symbols $\mathscr{E}_{h}^{T}$ denote the truncated expectations with respect to a measure with covariance $g^{(h)}$. We have that $\mathcal{O}$ is equal to $\mathscr{L}$ only if $v=v_{0}$ and the tree contributes to the local part of the effective potential. We also associate to each field a labels, $f, f=1, \ldots, n_{\tau}$, where $n_{\tau}$ is the number of the fields associated with all the endpoints of the tree. To every field with label $f$ corresponds a momentum $k(f)$ and the indices $\vec{\omega}(f), \sigma(f), \varepsilon(f)= \pm 1$ and, also, the index $s(f)=0,1,2$ allowing us to distinguish the three possibilities $\psi_{k(f), \vec{\omega}(f), \sigma(f)}^{\varepsilon(f)}, E(\vec{k}) \psi_{k(f), \vec{\omega}(f), \sigma(f)}^{\varepsilon(f)},-i k_{0} \psi_{k(f), \vec{\omega}(f), \sigma(f)}^{\varepsilon(f)}$. We call $I_{v_{0}}$ the set of $f$ labels.

It is possible to check that the effective potential Eq. (25) can be written as 


$$
V^{(k)}\left(\psi^{(\leqq k)}\right)=\sum_{n=1}^{\infty} \sum_{\tau \in \tau_{n}} V^{(k)}\left(\tau, \psi^{(\leqq k)}\right) .
$$

From Eq. (26) we see that each set of running coupling constants $\vec{v}_{h}$ is determined once that a set $\vec{v}_{0}$ is given from the relation $\vec{v}_{h-1}=\vec{v}_{h}+\beta_{h}\left(\vec{v}_{h}, \vec{v}_{h+1}, \ldots \vec{v}_{0}\right)$, where $\beta$, called beta function, is a sum over all the trees contributing to the relevant part of the effective potential. We define

$$
V^{(k)}\left(\tau, \psi^{(\leqq k)}\right)=\int d k_{v_{0}} \sum_{P_{v_{0}}} V^{(k)}\left(\tau, P_{v_{0}}, k_{v_{0}}\right) \tilde{\psi}^{(\leqq k)}\left(P_{v_{0}}\right) \delta\left(\sum_{f \in P_{v_{0}}} \varepsilon(f) k(f)+2 n \pi / a\right),
$$

where $k_{v_{0}}$ is the set of all the momentum variables of the $n_{\tau}$ fields, $P_{v_{0}}$ is a nonempty subset of $I_{v_{0}},\left|P_{v_{0}}\right|$ are the number of elements of this subset, $\sum_{P_{\iota_{0}}}$ is the sum over such subsets and $\tilde{\psi}^{(\leqq k)}\left(P_{v_{0}}\right)=\prod_{f \in P_{\iota_{0}}} \psi_{k(f), \vec{\omega}(f), \sigma(f), s(f)}^{\varepsilon(f),}$.

If in Eq. (26) we expanded the expectations by Wick's theorem, we could represent the r.h.s. as a sum of Feynaman graphs (see [B.G., B.G.P.S.]). A Feynman graph is constructed by symbolising the fields associated with every end-point of the tree as oriented half lines emerging from that point and enclosing the endpoints belonging to the cluster $v$ together with their half lines into a ideal box. We pair, i.e. contract, the half lines in internal lines (all but the external lines $\left.\tilde{\psi}^{(\leqq k)}\left(P_{v_{0}}\right)\right)$ and we associate to each of them a propagator $g^{h_{v}}$, if the line is contained in the ideal box containing the cluster $v$ and not in any one with higher frequency. Every graph contributes to the effective potential with a term of the form $\int d k^{P_{v_{0}}} W_{h_{v_{0}}}\left(k^{P_{\iota_{0}}}\right) \delta\left(\sum_{f \in P_{\iota_{0}}} \varepsilon(f) k(f)+2 n \pi / a\right) \tilde{\psi}^{(\leqq k)}\left(P_{v_{0}}\right)$, where $k^{P_{\iota_{0}}}$ is the set of the variables $k(f)$ with $f \in P_{v_{0}}$ and $W$, called value of the graph, is the product of the propagators of the graph and of the running couplings or the kernels in Theorem 2.1 associated to the end points, integrated over the momenta of the internal lines.

Furthermore, if $G_{\tau}$ is the set of all Feynman graphs associated with $\tau$, given $g \in$ $G_{\tau}$, it is natural to associate a subgraph $g_{v}$ to the vertex $v$ enclosing into an ideal box the cluster $v$ and cutting into half lines the lines connecting points in the $v$ cluster with points outside from it. Each $g_{v}$ is of the form $\int d k^{P_{\iota}} W_{h_{v}}\left(k^{P_{v}}\right) \delta\left(\sum_{f \in P_{l}} \varepsilon(f) k(f)\right.$ $+2 n \pi / a) \bar{\psi}^{\left(\leqq h_{l}-1\right)}\left(P_{v}\right)$ where $\bar{\psi}^{\left(\leqq h_{v}-1\right)}\left(P_{v}\right)$ are the half lines emerging from $v$ before contraction and $P_{v}$ is defined as $P_{v_{0}}$. On all this term the $\mathscr{R}$ operation acts, if $v \neq v_{0}$, while if $v \equiv v_{0}$ the operation $\mathscr{L}$ or $\mathscr{R}$ acts, depending on whether it contributes to the relevant or to the irrelevant part of the effective potential. It is convenient to write $W(k)$ as a function of $k^{\prime}=k-\vec{\omega} p_{F}$ introduced in the preceding section defining $W_{h_{l}}\left(k^{\prime P_{v}}+\vec{\omega}^{P_{v}} p_{F}\right)=f_{h_{r}}\left(k^{\prime P_{v}} ; \vec{\omega}^{P_{v}}\right)$.

We call scaling dimension $D\left(P_{v_{0}}\right)=-2+\sum_{A \in P_{\iota_{0}}}\left(1 / 2+\chi_{A}\right)$, where $\chi_{A}=0$ if $A=\psi_{k+\vec{\omega} p_{F}, \vec{\omega}, \sigma}^{h}, \chi_{A}=1$ if $A=E(k) \psi_{k^{\prime}+\vec{\omega} p_{F}, \vec{\omega}, \sigma}^{h}$ or $A=-i k_{0} \psi_{k^{\prime}+\vec{\omega} p_{F}, \vec{\omega}, \sigma}^{h}$. The size of a generic graph associated with a monomial $\tilde{\psi}^{\leqq h}\left(P_{v_{0}}\right)$ with value $W^{h}$ is defined by

$$
\left\|W^{h}\right\|=\sup _{k_{2}^{e x}, \ldots k_{n}^{e x}} \gamma^{h D\left(P_{v_{0}}\right)} d_{h}\left(k_{1}^{e x}\right) \ldots d_{h}\left(k_{n}^{e x}\right) W^{h}\left(k_{1}^{e x}, \ldots k_{n}^{e x}\right)
$$

where $d_{n}(k)$ is the characteristic function of the support of $h\left(\gamma^{-2 h+2}\left(k_{0}^{2}+E(\vec{k})^{2}\right)\right)$.

In order to motivate our definition of localization suppose for a moment that $\mathscr{R}=I$, where $I$ is the identity operator; by a standard calculation it is possible 
to prove that the size, Eq. (27), of a Feynman graph is bounded by $\|W\|<$ $c^{n} \varepsilon^{n} \prod_{v} \gamma^{-\left(h_{t}-h_{r^{\prime}}\right) D\left(P_{v}\right)}$, where $v^{\prime}$ is the vertex preceding $v$ in the tree ordering. To obtain an estimate of the perturbative contribution of order $n$ to the effective potential, we must sum over trees. In order to have an estimate uniform in $\beta, L$ it is necessary that $D\left(P_{v}\right)>0$ for all $P_{v}$. But we have that $D\left(P_{v}\right)=-1$ if $\left|P_{v}\right|=2$ and $\sum_{A \in P_{v}} \chi_{A}=0$, while $D\left(P_{v}\right)=0$ if $\left|P_{v}\right|=4$ and $\sum_{A \in P_{v}} \chi_{A}=0$ or $\left|P_{v}\right|=2$ and $\sum_{A \in P_{t}} \chi_{A}=1$. Like in [B.G.] one could define as "relevant part" of the effective potential the sum of its local quadratic and quartic parts in the fields. However such definitions would still contain irrelevant terms. This can be easily understood by remarking that for $h$ suitably small the contributions to the effective potential $V^{h}$ having forms:

$$
\begin{aligned}
& \int \prod_{i=1}^{4} d k_{i}^{\prime} f_{h_{i}}^{4}\left(k_{1}^{\prime}, k_{2}^{\prime}, k_{3}^{\prime}, k_{4}^{\prime} ; \underline{\vec{\omega}}\right) \delta\left(k_{1}^{\prime}+k_{2}^{\prime}-k_{3}^{\prime}-k_{4}^{\prime}+\left(\vec{\omega}_{1}+\vec{\omega}_{2}-\vec{\omega}_{3}-\vec{\omega}_{4}\right) p_{F}+\frac{2 n \pi}{a}\right) \\
& \psi_{k_{1}^{\prime}+\vec{\omega}_{1} p_{F}, \vec{\omega}_{1}, \sigma}^{+} \psi_{k_{2}^{\prime}+\vec{\omega}_{2} p_{F}, \vec{\omega}_{2}, \sigma^{\prime}}^{+} \psi_{k_{3}^{\prime}+\vec{\omega}_{3} p_{F}, \vec{\omega}_{3}, \sigma}^{-} \psi_{k_{4}^{\prime}+\vec{\omega}_{4} p_{F}, \vec{\omega}_{4}, \sigma}^{-} \\
& \quad \int d k_{1}^{\prime} d k_{2}^{\prime} \delta\left(k_{1}^{\prime}-k_{2}^{\prime}+\left(\vec{\omega}_{1}-\vec{\omega}_{2}\right) p_{F}+2 n \pi / a\right) f^{2}\left(k_{1}^{\prime}, k_{2}^{\prime} ; \underline{\vec{\omega}}\right) \psi_{k_{1}^{\prime}+\vec{\omega}_{1} p_{F}, \vec{\omega}_{1}, \sigma}^{+} \psi_{k_{2}^{\prime}+\vec{\omega}_{2} p_{F}, \vec{\omega}_{2}, \sigma}^{-}
\end{aligned}
$$

are vanishing unless $\left(\vec{\omega}_{1}+\vec{\omega}_{2}-\vec{\omega}_{3}-\vec{\omega}_{4}\right) p_{F}+\frac{2 n \pi}{a}=0$ in the first case and $\left(\vec{\omega}_{1}-\vec{\omega}_{2}\right) p_{F}+2 n \pi / a=0$ in the second as the delta's in the above equations cannot be satisfied for the support properties of the propagator (see Eq. (19) and relative discussion).

It is possible to check by a standard calculation that the size of the generic Feynman graph contributing to the effective potential defined above is bounded by

$$
\|W\|<C^{m} \varepsilon^{n} \prod_{v} \gamma^{-\left(h_{\iota}-h_{t^{\prime}}\right)\left(D\left(P_{c}\right)+z_{\iota}\right)}
$$

where $D\left(P_{v}\right)+z_{v}>0$ (the $\mathscr{R}$ operation was defined in order to make true such an inequality), $\varepsilon=\max \left|\vec{v}_{h}\right|$ and $c$ is a suitable constant independent from $n$. By repeating the estimates in [G.] it is easy to see that Eq. (28) implies that $\left|V^{(k)}\left(\tau, \psi^{\prime k}\right)\right| \leqq$ $\varepsilon^{n} c^{n} n$ !. We shall use (and prove) an equation stronger than Eq. (28), hence we do not dicuss its proof in more detail.

It is convenient to see the effect of $\mathscr{L}$ when $V^{k}(\psi \leqq k)$ is written as an integral over the coordinates. Writing

$$
\begin{gathered}
\int d k^{\prime P_{v}} \delta\left(\sum_{f \in P_{l}} \varepsilon(f) k(f)+\varepsilon(f) \vec{\omega}(f) p_{F}+2 n \pi / a\right) f_{h_{v}}\left(k^{\prime P_{v}} ; \vec{\omega}^{P_{l}}\right) \tilde{\psi}^{\left(\leqq h_{v}-1\right)}\left(P_{v}\right) \\
\quad=\int d x^{P_{v}} W\left(x^{P_{\imath}}\right) \hat{\psi}^{\left(\leqq h_{t}-1\right)}\left(P_{v}\right),
\end{gathered}
$$

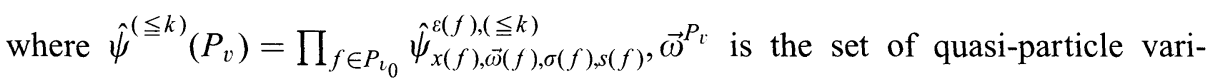
ables $\vec{\omega}(f)$ such that $f \in P_{v}$ and $W\left(x^{P_{v}}\right)$ is a not traslation invariant function unless $\sum_{f i n P_{l}} \varepsilon(f) \vec{\omega}(f) p_{F}+2 n \pi / a=0$ in which case it is translation invariant (see the 
delta-function in the above equation). By Eq. (22), (23) we see, by performing a Fourier transform, that the $\mathscr{L}$-operation acts in the following ways:

$$
\begin{gathered}
\mathscr{L} \int_{\Lambda} W_{h_{v}}\left(x_{1}, \ldots x_{n} ; \underline{\vec{\omega}}\right) \hat{\psi}_{x_{1}, \vec{\omega}_{1}, \sigma_{1}}^{+} \ldots \hat{\psi}_{x_{n}, \vec{\omega}_{n}, \sigma_{n}}^{-} d x_{1} \ldots d x_{n}=0 \quad n>4, \\
\mathscr{L} \int_{\Lambda} W_{h_{l}}\left(x_{1}-x_{4}, x_{2}-x_{4}, x_{3}-x_{4} ; \underline{\vec{\omega}}\right) \hat{\psi}_{x_{1}, \vec{\omega}_{1}, \sigma}^{+} \hat{\psi}_{x_{2}, \vec{\omega}_{2}, \sigma^{\prime}}^{+} \hat{\psi}_{x_{3}, \vec{\omega}_{3}, \sigma^{\prime}}^{-} \hat{\psi}_{x_{4}, \vec{\omega}_{4}, \sigma}^{-} \prod_{i=1}^{4} d x_{i}= \\
\delta_{\left(\vec{\omega}_{1}+\vec{\omega}_{2}-\vec{\omega}_{3}-\vec{\omega}_{4}\right) p_{F}+2 n \pi / a, 0} \int_{\Lambda} d t_{1} d t_{2} d t_{3} W_{h_{v}}\left(t_{1}, t_{2}, t_{3} ; \underline{\vec{\omega}}\right) s_{L, \beta}\left(t_{1}, t_{2}, t_{3}\right) \\
\times \int_{\Lambda} \delta\left(x_{1}-x_{2}\right) \delta\left(x_{2}-x_{3}\right) \delta\left(x_{3}-x_{4}\right) \hat{\psi}_{x_{1}, \vec{\omega}_{1}, \sigma}^{+}, \hat{\psi}_{x_{2}, \vec{\omega}_{2}, \sigma^{\prime}}^{+} \hat{\psi}_{x_{3}, \vec{\omega}_{3}, \sigma^{\prime}}^{-} \hat{\psi}_{x_{4}, \vec{\omega}_{4}, \sigma}^{-} \prod_{i=1}^{4} d x_{i},
\end{gathered}
$$

where $s_{L, \beta}\left(t_{1}, t_{2}, t_{3}\right)=\frac{1}{4} \sum_{j, k=1}^{2} e^{i \frac{\pi}{L}(-1)^{\prime}\left(\vec{t}_{1}+\vec{t}_{2}-\vec{t}_{3}\right)+i \frac{\pi}{\beta}(-1)^{k}\left(t_{1}^{0}+\vec{t}_{2}^{0}-\vec{t}_{2}^{0}\right)}$ and is present only because we are studying the sytem with finite volume and with temperature different from zero; moreover

$$
\begin{aligned}
& \mathscr{L} \int_{\Lambda} d x_{1} d x_{2} \hat{\psi}_{x_{1}, \vec{\omega}_{1}, \sigma}^{+} \hat{\psi}_{x_{2}, \vec{\omega}_{2}, \sigma}^{-} W_{h_{v}}\left(x_{1}-x_{2} ; \underline{\vec{\omega}}\right)=\delta_{\vec{\omega}_{1}, \vec{\omega}_{2}} \int_{\Lambda} d t W_{h_{v}}(t ; \vec{\omega}) \cos \left(\frac{\pi}{L} \vec{t}\right) \cos \left(\frac{\pi}{\beta} t_{0}\right) \\
& \quad \times \int_{\Lambda} d x_{1} d x_{2} \delta\left(x_{1}-x_{2}\right) \hat{\psi}_{x_{1}, \vec{\omega}, \sigma}^{+} \hat{\psi}_{x_{2}, \vec{\omega}, \sigma}^{-} \\
& \quad+\int_{\Lambda} d t W_{h_{v}}(t ; \vec{\omega}) \frac{\beta}{\pi} \sin \left(t_{0} \frac{\pi}{\beta}\right) \cos \left(\frac{\pi}{L} \vec{t}\right) \int_{\Lambda} d x_{1} d x_{2} \partial_{x_{2}^{0}} \delta\left(x_{1}-x_{2}\right) \hat{\psi}_{x_{1}, \vec{\omega}, \sigma}^{+} \hat{\psi}_{x_{2}, \vec{\omega}, \sigma}^{-} \\
& \quad+\int_{\Lambda} d t W_{h_{v}}(t ; \vec{\omega}) \frac{L}{\pi} \sin \left(\vec{t} \frac{\pi}{L}\right) \cos \left(\frac{\pi}{\beta} t_{0}\right) \int_{\Lambda} d x_{1} d x_{2} \bar{\partial}_{\vec{x}_{2}, \vec{\omega}} \delta\left(x_{1}-x_{2}\right) \hat{\psi}_{x_{1}, \vec{\omega}, \sigma}^{+} \hat{\psi}_{x_{2}, \vec{\omega}, \sigma}^{-},
\end{aligned}
$$

where the kernels $W$ are antiperiodic functions both in the time and space variables with period $\beta$ and $L, t=\left(t^{0}, \vec{t}\right), \bar{\partial}_{\vec{x}, \vec{\omega}}$, the covariant derivative, is defined by

$$
\bar{\partial}_{\vec{x}, \vec{\omega}} f(\vec{x})=i \sum_{\vec{k}} \vec{\omega} E\left(\vec{k}+\vec{\omega} p_{F}\right) \hat{f}(\vec{k})
$$

If $\delta\left(x_{i}-x_{j}\right)$ are integrated away in the r.h.s. of Eq. (29), (30),(31) we see that the action of $\mathscr{L}$ has the effect that the monomials in the fields are changed into local expressions. This is the main reason for which we introduced the fields $\hat{\psi}_{x, \vec{\omega}, \sigma}^{\sigma}$ rather than working with $\psi_{x, \vec{\omega}, \sigma}^{\sigma}$ Eq. (4). The non-trivial action of $\mathscr{R}$ on the terms with four external lines is

$$
\begin{aligned}
& \int_{\Lambda} \mathscr{R} W_{h_{v}}\left(x_{1}-x_{4}, x_{2}-x_{4}, x_{3}-x_{4} ; \underline{\vec{\omega}}\right) \hat{\psi}_{x_{1}, \vec{\omega}_{1}, \sigma}^{+} \hat{\psi}_{x_{2}, \vec{\omega}_{2}, \sigma^{\prime}}^{+} \hat{\psi}_{x_{3}, \vec{\omega}_{3}, \sigma^{\prime}}^{-} \hat{\psi}_{x_{4}, \vec{\omega}_{4}, \sigma}^{-} \prod_{i=1}^{4} d x_{i}= \\
& \int_{\Lambda}^{4} d \prod_{i=1}^{4} d x_{l} \hat{\psi}_{x_{1}, \vec{\omega}_{1}, \sigma}^{+} \hat{\psi}_{x_{2}, \vec{\omega}_{2}, \sigma^{\prime}}^{+} \hat{\psi}_{x_{3}, \vec{\omega}_{3}, \sigma^{\prime}}^{-} \hat{\psi}_{x_{4}, \vec{\omega}_{4}, \sigma}^{-}\left\{W_{h_{v}}\left(x_{1}-x_{4}, x_{2}-x_{4}, x_{3}-x_{4} ; \underline{\vec{\omega}}\right)\right. \\
& \left.-\delta\left(x_{1}-x_{2}\right) \delta\left(x_{2}-x_{3}\right) \delta\left(x_{3}-x_{4}\right) \int_{\Lambda} d t_{1} d t_{2} d t_{3} s_{L, \beta}\left(t_{1}, t_{2}, t_{3}\right) W_{h_{v}}\left(t_{1}, t_{2}, t_{3} ; \underline{\vec{\omega}}\right)\right\} .
\end{aligned}
$$


In the estimates of the following section it is convenient integrating $\delta^{\prime} s$ in Eq. (32) obtaining a different, equivalent form:

$$
\begin{aligned}
\int \mathscr{R} W & \left(x_{1}-x_{4}, x_{2}-x_{4}, x_{3}-x_{4} ; \underline{\vec{\omega}}\right) \hat{\psi}_{x_{1}, \vec{\omega}_{1}, \sigma}^{+} \hat{\psi}_{x_{2}, \vec{\omega}_{2}, \sigma^{\prime}}^{+} \hat{\psi}_{x_{3}, \vec{\omega}_{3}, \sigma^{\prime}}^{-} \hat{\psi}_{x_{4}, \vec{\omega}_{4}, \sigma}^{-} \prod_{i=1}^{4} d x_{i} \\
= & \int \prod_{i=1}^{4} d x_{l} W\left(x_{1}-x_{4}, x_{2}-x_{4}, x_{3}-x_{4} ; \underline{\vec{\omega}}\right)\left(1-s_{L, \beta}\left(\vec{x}_{1}-\vec{x}_{4}, x_{2}-x_{4}, x_{3}-x_{4}\right)\right) \\
& \times \hat{\psi}_{x_{1}, \vec{\omega}_{1}, \sigma}^{+} \hat{\psi}_{x_{1}, \vec{\omega}_{2}, \sigma^{\prime}}^{+} \hat{\psi}_{x_{1}, \vec{\omega}_{3}, \sigma^{\prime}}^{-} \hat{\psi}_{x_{1}, \vec{\omega}_{4}, \sigma}^{-}+ \\
& \int \prod_{i=1}^{4} d x_{i} W\left(x_{1}-x_{4}, x_{2}-x_{4}, x_{3}-x_{4} ; \underline{\vec{\omega}}\right) \cdot\left\{\hat{\psi}_{x_{1}, \vec{\omega}_{2}, \sigma}^{+} \hat{\psi}_{x_{2}, \vec{\omega}_{2}, \sigma^{\prime}}^{+} \hat{\psi}_{x_{3}, \vec{\omega}_{3}, \sigma^{\prime}}^{-} \hat{\psi}_{x_{4}, \vec{\omega}_{4}, \sigma}^{-}\right. \\
& \left.-1 / 2 \sum_{i=1}^{2} \hat{\psi}_{x_{l}, \vec{\omega}_{1}, \sigma}^{+} \hat{\psi}_{x_{i}, \vec{\omega}_{2}, \sigma^{\prime}}^{+} \hat{\psi}_{x_{l}, \vec{\omega}_{3}, \sigma^{\prime}}^{-} \hat{\psi}_{x_{i}, \vec{\omega}_{4}, \sigma}^{-}\right\}
\end{aligned}
$$

where $s_{L, \beta}\left(t_{1}, t_{2}, t_{3}\right)$ is, once again, present only because one wants to distinguish carefully the $L, \beta<\infty$ from the $L, \beta=\infty$ case. Equation (33) shows that there are two ways in which the renormalization acts on a subgraph with four half lines connecting points in the cluster $v$ to points outside it. One way renormalization affects the graphical analysis is that one of the half-lines does not represnt a $\psi$-field as we can write:

$$
\begin{aligned}
& \left(\hat{\psi}_{x_{1}, \vec{\omega}_{1}}^{+} D_{x_{2,1}, \vec{\omega}_{1}}^{+} \hat{\psi}_{x_{3}, \vec{\omega}_{3}}^{-} \hat{\psi}_{x_{4}, \vec{\omega}_{4}}^{-}+\hat{\psi}_{x_{1}, \vec{\omega}_{1}}^{+} \hat{\psi}_{x_{2}, \vec{\omega}_{2}}^{+} D_{x_{3,1}, \vec{\omega}_{3}}^{-}+\hat{\psi}_{x_{1}, \vec{\omega}_{1}}^{+} \hat{\psi}_{x_{2}, \vec{\omega}_{2}}^{+} \hat{\psi}_{x_{3}, \vec{\omega}_{3}}^{-} D_{x_{4,1}, \vec{\omega}_{4}}^{-}\right. \\
& +D_{x_{1,2}, \vec{\omega}_{1}}^{+} \hat{\psi}_{x_{1}, \vec{\omega}_{1}}^{+} \hat{\psi}_{x_{3}, \vec{\omega}_{3}}^{-} \hat{\psi}_{x_{4}, \vec{\omega}_{4}}^{-}+\hat{\psi}_{x_{1}, \vec{\omega}_{1}}^{+} \hat{\psi}_{x_{2}, \vec{\omega}_{2}}^{+} D_{x_{3,2}, \vec{\omega}_{3}}^{-} \hat{\psi}_{x_{4}, \vec{\omega}_{4}}^{-}+\hat{\psi}_{x_{1}, \vec{\omega}_{1}}^{+} \hat{\psi}_{x_{2}, \vec{\omega}_{2}}^{+} \hat{\psi}_{x_{3}, \vec{\omega}_{3}}^{-} D_{x_{4,2}, \vec{\omega}_{4}}^{-},
\end{aligned}
$$

where

$$
\begin{gathered}
D_{x_{j, i, \vec{\omega}}}^{\varepsilon}=\hat{\psi}_{x_{l}, \vec{\omega}}^{\varepsilon}-\hat{\psi}_{x_{\jmath}, \vec{\omega}}^{\varepsilon}=\left(x_{i}-x_{j}\right) \int_{0}^{1} d u \partial \hat{\psi}_{x_{J, l}^{\prime}(u), \vec{\omega}}^{\varepsilon}, \\
x_{j, i}^{\prime}(u)=u x_{j}+(1-u) x_{j}, \quad \partial=\left(\partial_{t}, \partial_{\vec{\omega}}\right) .
\end{gathered}
$$

$x_{j, i}^{\prime}(u)$ are called interpolated points. It is easy to check that the effect of this substitution (i.e. a $D$-line instead of a $\psi$-line) is that in the estimate of a generic Feynman graph, in which the line representing $D$-field has an end in the cluster $v$ and the other in cluster the $v^{\prime}$, there is an extra factor $\gamma^{-\left(h_{t}-h_{t^{\prime}}\right)}$ with respect to the case in which $\mathscr{R}=1$.

The other way in which $\mathscr{R}$ can act, from Eq. (33) is that the kernel $W$ is substituted by a kernel $W(1-s)$ and this produces, in the estimate, at least an extra factor $\gamma^{-\left(h-h^{*}\right)}$ with respect to the not renormalized case, with $h^{*}=\min \left(h_{L}, h_{\beta}\right)$, where $h_{L}$ is such that if $h<h_{L} g_{\vec{\omega}}^{h} \equiv 0$ for $\vec{k}$ of the form $2 n \pi / L, n$ integer. Of course $\gamma^{-h_{L}}=$ const $L$. We define $h_{\beta}$ in the same way. Of course if $h \leqq h^{*}$ and $V^{h}(\phi)=-\log \int P\left(d \psi^{h+1}\right) e^{-V^{h+1}\left(\psi^{h+1}+\phi\right)}$, then

$$
V^{h}(\phi)=V^{h-1}(\phi)
$$

that is the effective potential stops flowing. Equation (36) is the analogue of Lemma 1 in [B.G.P.S.] whose proof (not explicitly written there) is not so trivial for the exponential decay of the propagators in momentum space. Since there are no non-trivial vertex with $h^{\prime} \leqq h^{*}$, then we have that $\gamma^{-\left(h_{v}-h^{*}\right)} \leqq \gamma^{-\left(h_{v}-h_{r^{\prime}}\right)}$. Similar considerations can be made on the terms with two external lines. 


\section{Analyticity of the Anomalous Effective Potential}

In order to see if the flow of the relevant running coupling $\vec{v}_{h}$ (see lines following Eq. (25)) is bounded we write then the equations for the $\vec{v}_{h}$ up to the second order:

$$
\begin{aligned}
g_{1, h-1} & =g_{1, h}-2 \beta g_{1, h}^{2}+O\left(\gamma^{h}\right), \\
g_{2, h-1} & =g_{2, h}-\beta g_{1, h}^{2}+O\left(\gamma^{h}\right), \\
g_{4, h-1} & =g_{4, h}+O\left(\gamma^{h}\right), \\
\alpha_{h-1} & =\alpha_{h}+\tilde{\beta}_{1} g_{1, h}^{2}+\tilde{\beta}_{2} g_{2, h}^{2}+O\left(\gamma^{h}\right), \\
\zeta^{h-1} & =\zeta^{h}+\tilde{\beta}_{1} g_{1, h}^{2}+\tilde{\beta}_{2} g_{2, h}^{2}+O\left(\gamma^{h}\right), \\
v^{h-1} & =\gamma v^{h}+O\left(\gamma^{h}\right),
\end{aligned}
$$

where $\beta, \tilde{\beta}_{1}, \tilde{\beta}_{2}>0$ and the equation for $t_{h}$ (see lines following Eq. (25)) is not written for simplicity as $\vec{v}_{h}$ does not depend on $t_{h}$. We have supposed that $p_{F} \neq \pi / 2 a$ postponing the discussion of the case $p_{F}=\pi / 2 a$ to the last section. These equations are qualitatively similar to the equation founded by Solyom [S.] for his phenomenological hamiltonian. If $g_{1,0}<0$ and the corrections $O\left(\gamma^{h}\right)$ are neglected, then $g_{1, h}$ grows so that the second order truncation has no meaning. If $g_{1,0} \geqq 0$ things looks different. It is easy to check by using the general methods of stability theory that, for $v_{0}$ small enough:

$$
\begin{gathered}
g_{1, h} \simeq \frac{g_{1,0}}{1-2 \beta h g_{1,0}} \rightarrow 0, \quad g_{4, h}=g_{4,0}, \\
g_{2, h} \rightarrow g_{2, \infty} \simeq g_{2,0}-\beta \sum_{h=0}^{-\infty} \frac{g_{1,0}^{2}}{\left(1-2 \beta h g_{1,0}\right)^{2}}=g_{2,0}+O\left(g_{1,0}^{2}\right) .
\end{gathered}
$$

However also in this case the flow is unbounded. In fact we have that $\alpha_{h}=$ $\alpha_{0}+\sum_{h=-\infty}^{0}\left(\tilde{\beta}_{1} g_{1, h}^{2}+\tilde{\beta}_{2} g_{2, h}^{2}\right)$ and similarly for $\zeta_{h}$; so that in any case $\alpha_{h}, \zeta_{h} \rightarrow \infty$ because $g_{2, h}, g_{4, h}$ do not go to zero. Note that even if the third order contribution to the Beta function makes that $g_{2, h}, g_{4, h}$ tend to zero, this would happen very slowly, i.e. not faster than $1 / \sqrt{-h}$.

This suggests that we try a new and more general scaling approach, including the one described in the preceding section, the anomalous scaling. Given a sequence of positive numbers $Z_{h}$, with $Z_{0}=1$ we can write, for $h \leqq 0$,

$$
\int P z_{h+1}\left(d \psi^{(\leqq h)}\right) e^{-\bar{V}^{(h)}\left(\sqrt{Z_{h+1}} \psi^{(\leqq h)}\right)}=\int \tilde{P}_{Z_{h}}\left(d \psi^{(h)}\right) P_{Z_{h}}\left(d \psi^{(<h)}\right) e^{-V^{(h)}\left(\sqrt{Z_{h}} \psi^{(\leqq h)}\right)},
$$

where $P_{Z_{h}}\left(d \psi^{(h)}\right)$ denotes the Grassmannian integration with propagator $\frac{g^{h}}{Z_{h}}$ and $\tilde{P}_{Z_{h}}\left(d \psi^{(h)}\right)$ the one with propagator $\frac{\tilde{g}^{h}}{Z_{h}}$ where, if we call $C_{h}(k)=\sum_{k=-\infty}^{h} f\left(\gamma^{-2 k+2}\right.$ $\left.\left(k_{0}^{2}+E(\vec{k})^{2}\right)\right), \tilde{g}^{(h)}(k)$ is given by $g^{(h)}(k)+\tau^{(h)}(k)$ with:

$$
\tau^{(h)}(k)=\frac{C_{h}(k)\left(1-C_{h}(k)\right)}{-i k_{0}+E(\vec{k})} \frac{z_{h}}{1+z_{h} C_{h}(k)} .
$$


Fermi Surface for a $\mathrm{d}=1$ Interacting Fermions in Periodic Potential

$\tilde{V}^{h}(\psi)$ is determined from $V^{h+1}(\psi)$ using the following relation, for $h<0$ :

$$
e^{-\tilde{V}^{(h)}\left(\sqrt{Z_{h+1}} \psi^{(\leqq h)}\right)}=\int \tilde{P}_{Z_{h+1}}\left(d \psi^{(h+1)}\right) e^{-V^{(h+1)}\left[\sqrt{Z_{h+1}}\left(\psi^{(h+1)}+\psi^{(\leqq h)}\right)\right]} .
$$

Note that $\tilde{V}^{0}(\psi)=V^{0}(\psi)$ and that the relevant part of $\tilde{V}^{h}(\psi)$ (we write in it also the constant part of $\left.\tilde{V}^{h}(\psi \leqq h)\right)$ is given by

$$
\mathscr{L} \tilde{V}^{h}\left(\sqrt{Z_{h+1}} \psi^{\leqq h}\right)=Z_{h+1} \gamma^{h} n_{h} F_{v}+Z_{h+1} a_{h} F_{\alpha}+Z_{h+1} z_{h} F_{\zeta}+Z_{h+1}^{2} \sum_{i=1}^{4} \tilde{g}_{l}^{h} F_{i}+t_{h}|\Lambda|,
$$

where $|\Lambda|=L \beta$. The sequence of $Z_{h}$ is chosen so that the relevant part of $V^{h}(\psi)$ does not contain the term proportional to $F_{\zeta}$, i.e.

$$
\mathscr{L} V^{h}\left(\sqrt{Z_{h}} \psi^{\leqq h}\right)=Z_{h} \gamma^{h} v_{h} F_{v}+Z_{h} \delta_{h} F_{\alpha}+Z_{h}^{2} \sum_{l=1}^{4} g_{i}^{h} F_{l}+\gamma^{2 h} \theta_{h}|\Lambda|,
$$

and this is achieved by taking $Z_{h}=Z_{h+1}\left(1+z_{h}\right)$. Clearly we have $v_{h}=\frac{Z_{h+1}}{Z_{h}} n_{h}$, $g_{h}=\left(\frac{Z_{h+1}}{Z_{n}}\right)^{2} \tilde{g}^{h}, \quad \delta_{h}=\frac{Z_{h+1}}{Z_{h}}\left(a_{h}-z_{h}\right), \quad \gamma^{2 h} \theta_{h}=t_{h}+t_{h}^{\prime}, \quad$ where $\quad t_{h}^{\prime}=\int \frac{d k}{(2 \pi)^{2}}$ $\log \left(1+\frac{Z_{h}-Z_{h+1}}{Z_{h+1}} C_{h}(k)\right) \cdot V^{h}$ is called anomalous effective potential.

We can write $V^{(k)}\left(\hat{\psi}^{(\leqq k)}\right)=\sum_{n=1}^{\infty} \sum_{\tau \in \tau_{n}} \tilde{V}^{(k)}\left(\tau, \hat{\psi}^{(\leqq k)}\right)$ with

$$
\left.\tilde{V}^{(k)}\left(\tau, Z_{k}^{\frac{1}{2}} \psi^{(\leqq k)}\right)=\mathcal{O} \frac{1}{s_{v_{0}} !} E_{k+1}^{T}\left[V^{k+1)}\left(\tau^{1}, Z_{k+1}^{\frac{1}{2}} \psi^{(\leqq k+1)}\right), \ldots\right)\right],
$$

where $n \geqq 2, \tau^{1} \ldots \tau^{s_{0}}$ are the subtrees starting from $v_{0}$ (the first vertex above the root), the symbols $E_{h}, E_{h}^{T}$ denote the expectations with respect to a grassmannian integration with propagator $Z_{h}^{-1} \tilde{g}^{(h)}$ and $\mathcal{O}$ is equal to $\mathscr{L}^{*}$, if the tree contributes to the local part of the potential, or $\mathscr{R}$, if it contributes to the irrelevant part, where $V^{h}(\psi)=\mathscr{L}^{*} \tilde{V}^{(h)}\left(\frac{Z_{h+1}}{Z_{h}} \psi\right)+\mathscr{R} \tilde{V}^{(h)}\left(\frac{Z_{h+1}}{Z_{h}} \psi\right)$ and $\mathscr{L}^{*} \tilde{V}^{(h)}$ differs from $\mathscr{L} \tilde{V}^{(h)}$ only because it does not contain the addend proportional to $-i k_{0} \psi_{k, \sigma, \vec{\omega}}^{+} \psi_{k, \sigma, \vec{\omega}}^{-}$.

We write the effective potential as an integral in position space, i.e. we write:

$$
V^{(k)}\left(\tau, \sqrt{Z_{k}} \psi^{(\leqq k)}\right)=\int d x_{v_{0}} \sum_{P_{v_{0}}}\left(Z_{k}\right)^{\frac{1}{2}\left|P_{\iota_{0}}\right|} V^{(k)}\left(\tau, P_{v_{0}}, x_{v_{0}}\right) \psi^{(\leqq k)}\left(P_{v_{0}}\right)
$$

where $x_{v_{0}}$ is the set of all the coordinate variables. We define also the kernels

$$
W^{(k)}\left(\tau, P_{v_{0}}, x^{P_{v_{0}}}\right)=\int_{\Lambda} d\left(x_{v_{0}} \backslash x^{P_{\iota_{0}}}\right) V^{(k)}\left(\tau, P_{v_{0}}, x_{v_{0}}\right)
$$

so that

$$
V^{(k)}\left(\tau, Z_{k}^{\frac{1}{2}} \psi^{(\leqq k)}\right)=\sum_{P_{\iota_{0}}} \int_{\Lambda} d x^{P_{v_{0}}} W^{(k)}\left(\tau, P_{v_{0}}, x^{P_{v_{0}}}\right) Z_{k}^{\frac{1}{2}\left|P_{v_{0}}\right|} \tilde{\psi}^{(\leqq k)}\left(P_{v_{0}}\right)
$$

Here $x^{P_{v_{0}}}$ is the set of points on which the monomial $\tilde{\psi}^{(\leqq k)}\left(P_{v_{0}}\right)$ depends (recall that there can be more than one point for each field). Let us define: $\vec{v}_{h}=\left(g_{h}^{\alpha}, \delta_{h}, v_{h}\right)$ 
and $\varepsilon_{k}=\max _{i, h \geqq k}\left|v_{i, h}\right|$ and let us formulate the following theorem:

Theorem 4.1. There exist a constant $\bar{\varepsilon}>0$, such that, if $\varepsilon_{k} \leqq \bar{\varepsilon}$ and $\sup _{k<h^{\prime}<h}$ $\left|\frac{Z_{h}}{Z_{h^{\prime}}}\right| \leqq 1+c_{2} \bar{\varepsilon}^{2}, c_{2}>0$ then, for every $N$ :

$$
\int_{\Lambda} d\left(x^{\left(P_{\iota_{0}}\right)}\right)\left(1+\gamma^{k} d\left(P_{v_{0}}\right)\right)^{N} \sum_{\tau \in \tau_{n}}\left|W\left(\tau, P_{v_{0}}, x^{P_{v_{0}}}\right)\right| \leqq \Lambda \gamma^{-k D\left(P_{v_{0}}\right)}\left(C_{N} \varepsilon_{k}\right)^{n},
$$

where $d\left(P_{v_{0}}\right)$ is the length of the shortest tree graph connecting the set of points $x^{\left(P_{v_{0}}\right)}, C_{N}$ is a constant and $D\left(P_{v_{0}}\right)$ is the scaling dimension of the monomial $\psi \leqq k\left(P_{v_{0}}\right)$.

Proof. Let be $\mathscr{E}_{h_{\iota}}^{T}\left(\tilde{\psi}\left(P_{1}\right), \ldots, \tilde{\psi}\left(P_{k}\right)\right)$ the truncated expectation with propagator $\tilde{g}^{\left(h_{\iota}\right)}$ of $n$ fields. By using a well known expansion of truncated expectation in terms of interpolating parameters $s_{t}, t=1, \ldots, k-1$ [Le., B.G.P.S.], we can write:

$$
\mathscr{E}_{h_{v}}^{T}\left(\tilde{\psi}\left(P_{1}\right), \ldots, \tilde{\psi}\left(P_{k}\right)\right)=\sum_{T_{l}} \prod_{l \in T_{v}} \tilde{g}^{\left(h_{l}\right)}\left(x_{l}-y_{l}\right) \int d P_{T_{v}}(s) \operatorname{det} G^{T_{v}}(s),
$$

where $T_{v}$ is an anchored tree graph between the clusters of space vertices from which the fields labeled with $P_{1}, \ldots, P_{k}$ emerge: this means that $T_{v}$ is a set of lines connecting two points in different clusters, which become a tree graph if one identifies all the points in the same cluster; we call $T=\cup_{v} T_{v}$ and, if $l \in$ $T_{v}, x_{l}, y_{l}$ are the end-points of the line and are such that $x_{l} \equiv x_{i, j}$ or $y_{l} \equiv x_{i^{\prime}, j^{\prime}}$, where $x_{l, j}$ is the coordinate of the $i^{\text {th }}$ field of the $j^{\text {th }}$ monomial $\tilde{\psi}\left(P_{j}\right) \cdot G^{T_{v}}(s)$ is a $(n-k+1) \times(n-k+1)$ matrix whose elements are $G_{j i j^{\prime} i^{\prime}}^{T_{v}}=S_{j j^{\prime}} \tilde{g}^{\left(h_{v}\right)}\left(x_{l^{\prime}, j^{\prime}}-x_{i, j}\right)$ with $x_{i^{\prime}, j^{\prime}}-x_{i, j}$ not belonging to $T_{v}, S_{j J^{\prime}}=\prod_{t=j}^{j^{\prime}-1} s_{t}$ and $d P_{T_{v}}(s)$ is a normalised measure which depends on $s_{t}$ and $T_{v}$.

From Eq. (41) and App. 2 we have that

$$
\begin{aligned}
V^{(k)}\left(\tau, P_{v_{0}}, x_{v_{0}}\right)= & \sum_{\left\{P_{v}\right\} \text { v not e.p. }} \prod\left(\frac{Z_{h_{v}}}{Z_{h_{v}-1}}\right)^{\frac{1}{2}\left|P_{v}\right|} \frac{1}{s_{v} !} \tilde{\mathscr{E}}_{h_{\iota}}^{T}\left(\tilde{\psi}\left(P_{v^{1}} / Q_{v^{1}}\right), \ldots,\right. \\
& \left.\tilde{\psi}\left(P_{v^{s_{v}}} / Q_{v^{s_{v}}}\right)\right) \prod_{\text {vnot e.p. }} \vec{v}_{h_{v}},
\end{aligned}
$$

where $\prod_{v \text { not e.p. }} \vec{v}_{h_{v}}$ is the product of the running couplings associated to the end points of the tree $\tau$ (which are $n$ if $\tau \in \tau_{n}$ ), $P_{v}$ is a not empty subset of $I_{v}$, the field labels reachable from $v, v_{i}$ are the $s_{v}$ vertices immediately following $v, \sum_{P_{v}}$ represents the sum over all the compatible choices of the subsets $P_{v}$ such that $Q_{v} \subset P_{v}, P_{v}=\cup Q_{v_{i}}$ and $\tilde{\mathscr{E}}_{h_{v}}^{T}$ obeys to an equation like Eq. (42) in which $\tilde{g}^{\left(h_{v}\right)}\left(x_{l}-y_{l}\right)$ is replaced by $\left|\xi_{l}-h_{l}\right|^{z_{l}} \tilde{g}^{\left(h_{l}\right)}\left(\xi_{l}-\eta_{l}\right)$, where $z_{l}$ is a positive integer $z_{l} \leqq 2$ and such that $\sum_{l \in T_{v}} z_{l} \leqq 2$. The end points of the lines in $T_{v} \xi_{l}, \eta_{l}$ can be simple points $x_{j, l}$ or interpolated points $x_{j, i}^{\prime}$ (see Eq. (35)), i.e

$$
x_{J, l}^{\prime}=\sum_{i^{\prime}} \varepsilon_{i^{\prime}, j}(\underline{u}) x_{i^{\prime}, j}, \quad \sum_{i^{\prime}} \varepsilon_{l^{\prime}, j}(\underline{u})=1, \quad \varepsilon_{i^{\prime}, j}(0)=\delta_{i^{\prime}, i},
$$

and $G_{j i j^{\prime} i^{\prime}}^{T_{l}}=S_{j j^{\prime}} \tilde{g}^{\left(h_{v}\right)}\left(x_{\imath^{\prime}, j^{\prime}}^{\prime}-x_{i, j}^{\prime}\right)$ with $x_{i^{\prime}, j^{\prime}}^{\prime}-x_{i, j}^{\prime}$ not belonging to $T_{v}$. 
Estimating the determinant in Eq. (42) by the Grahm-Hadamard (see for instance [B.G.P.S.]) inequality it follows:

$$
\begin{aligned}
\left|V^{(k)}\left(\tau, P_{v_{0}}, x_{v_{0}}\right)\right| \leqq & \bar{\varepsilon}^{n} \sum_{\left\{P_{v}\right\} \text { v }} \prod_{\text {not e.p. }}\left(\frac{Z_{h_{v}}}{Z_{h_{\imath}-1}}\right)^{\frac{1}{2}\left|P_{v}\right|} C^{\left|Q_{v}\right|-\left|P_{v}\right|} \ldots J\left(\tau, P_{v_{0}}, x_{v_{0}}\right) \\
& \cdot \gamma^{\frac{h_{v}}{2} \sum_{J=0}^{2}(2 j+1) \sum_{l}\left(\left|P_{v^{\prime}}^{\prime}\right|-\left|Q_{v^{i}}^{\prime}\right|\right)},
\end{aligned}
$$

where $P_{v}^{J}$ denotes the subset of $P_{v}$ correspondent to field with a derivative index of order $j, Q_{v}^{j}$ is defined analogously and:

$$
J\left(\tau, P_{v_{0}}, x_{v_{0}}\right)=\left(\prod_{\text {not e. } p} \frac{1}{s_{v} !}\right) \int \prod_{v \text { not e.p }} \sum_{T_{v}} \prod_{l \in T_{v}} d \underline{u}\left|\xi_{l}-\eta_{l}\right|^{z_{l}} \tilde{g}^{\left(h_{l}\right)}\left(\gamma^{h_{v}}\left(\xi_{l}-\eta_{l}\right)\right) .
$$

The interpolated points and the terms $\left|\xi_{l}-\eta_{l}\right|$ are produced by the renormalization, see Eq. (35). In [B.G.P.S.] it is found an equation very similar to Eq. (44), except that in the equation analogous to Eq. (45) the terms $\left|\xi_{l}-\eta_{l}\right|$ produced by the renormalization would not coincide with the argument of the propagator belonging to the anchored tree $T_{v}$ and $z_{l}$ would be bounded by $2 n$. However the proof given in [B.G.P.S.] for the boundedness of the integral over the coordinates of the analogue of Eq. (44) would not apply here as it requires exponential decay for the propagator. The elimination of the latter condition is the main technical innovation that we develop in this section.

We can perform in Eq. (45) the change of variables $\xi_{l}-\eta_{l} \rightarrow y_{l}$ realised from the following linear system $y=A_{T}(\underline{u}) x$, where $A_{T}(\underline{u})$ is a square matrix $n \times n$ whose elements are functions of the interpolated parameters $u$, so that we can write:

$$
\begin{aligned}
& \int_{\Lambda} d x_{v_{0}}\left(1+\gamma^{k} d\left(P_{v_{0}}\right)\right)^{N} J\left(\tau, P_{v_{0}}, x_{v_{0}}\right) \\
& \quad=\sum_{T}\left(\prod_{v \text { not e.p }} \frac{1}{s_{v} !}\right) \int d \underline{u}\left|\operatorname{det} A_{T}(u)^{-1}\right| \prod_{l \in T} \int d y_{l}\left(1+\gamma^{k} d_{y}\left(P_{v_{0}}\right)\right)^{N}\left|y_{l}\right|^{z} \tilde{g}^{\left(h_{v}\right)}\left(\gamma^{h_{l}} y_{l}\right),
\end{aligned}
$$

where $d_{y}\left(P_{v_{0}}\right)$ is the length of the shortest tree graph expressed in terms of $y$ variables. In Appendix 3 it is proved that $\left|\operatorname{det} A_{T}(u)\right|=1$ so:

$$
\int_{\Lambda} d x_{v_{0}}\left(1+\gamma^{k} d\left(P_{v_{0}}\right)\right)^{N} J\left(\tau, P_{v_{0}}, x_{v_{0}}\right) \leqq|\Lambda| C^{2 n} \prod_{v} C^{\sum_{\imath}\left|P_{v^{i}}\right|-\left|Q_{v^{i}}\right|} \gamma^{-2 h_{\iota}\left(s_{v}-1\right)-z_{l} h_{\iota}}
$$

Then Eq. (44), (46) imply that

$$
\gamma^{k D\left(P_{t_{0}}\right)} \sum_{\tau \in \tau_{n}} \int_{\Lambda} d x_{v_{0}}\left(1+\gamma^{k} d\left(P_{v_{0}}\right)^{N}\left|V^{(k)}\left(\tau, P_{v_{0}}, x_{v_{0}}\right)\right| \leqq\left(C C^{\prime} \varepsilon_{k}\right)^{n}\right.
$$

so that the theorem is proved. We remark that, without the result of Appendix 2, one would obtain Eq. (48) with $C^{n}$ replaced by $C_{n}^{n}$, where $C_{n}$ would be a function of $n$ and $C_{n}$ could only be bounded by $n$ ! or worse. 


\section{The Flow of Renormalization Group}

The Beta function can be written as $\beta_{h}\left(\vec{v}_{h}, \ldots, \vec{v}_{0}\right)=\bar{\beta}_{h}\left(\vec{v}_{h} \ldots \vec{v}_{0}\right)+\gamma^{h} \hat{\beta}_{h}\left(\vec{v}_{h}, \ldots, \vec{v}_{0}\right)$. In the spinless case $v_{i, h}=\left\{v_{h}, \delta_{h}, Z_{h}, \lambda_{h}\right\}$ and the function $\bar{\beta}_{h}\left(\vec{v}_{h} \ldots \vec{v}_{0}\right)$ is the same as in the $U(\vec{x})=0$ case. So repeating the arguments in [B.G.M., B.G.P.S] it is possible to prove that the flow is bounded and anomalous so that Theorem 1.1 holds.

The spinning case is more involved. The equations for the running coupling can be written explicitly in the following way:

$$
\begin{aligned}
g_{1, h-1}= & \frac{Z_{h}^{2}}{Z_{h-1}^{2}}\left[g_{1, h}+g_{1, h}^{2}\left(-2 \beta+B_{1}\left(g_{\geqq h}, \delta_{\geqq h}, v_{\geqq h}\right)+\gamma^{h} R_{1}\left(g_{\geqq h}, \delta_{\geqq h}, v_{\geqq h}\right)\right],\right. \\
g_{2, h-1}= & \frac{Z_{h}^{2}}{Z_{h-1}^{2}}\left[g_{2, h}+g_{2, h} B_{2}\left(g_{2, \geqq h}, g_{4, \geqq h}, \delta_{\geqq h}, v_{\geqq h}\right)\right. \\
& \left.+g_{1, h}^{2}\left(-\beta+B_{3}\left(g_{\geqq h}, \delta_{\geqq h}, v_{\geqq h}\right)\right)+\gamma^{h} R_{2}\left(g_{\geqq h}, \delta_{\geqq h}, v_{\geqq h}\right)\right], \\
g_{4, h-1}= & \frac{Z_{h}^{2}}{Z_{h-1}^{2}}\left[\left(g_{4, h}+B_{4}\left(g_{2, \geqq h}, g_{4, \geqq h}, \delta_{\geqq h}, v_{\geqq h}\right)\right.\right. \\
& \left.+g_{1, h}^{2} B_{5}\left(g_{\geqq h}, \delta_{\geqq h}, v_{\geqq h}\right)+\gamma^{h} R_{3}\left(g_{\geqq h}, \delta_{\geqq h}, v_{\geqq h}\right)\right], \\
\delta_{h-1}= & \frac{Z_{h}}{Z_{h-1}}\left[\delta_{h}+\delta_{h} g_{h}^{2} B_{6}\left(g_{\geqq h}\right)+v_{h}^{2} B_{7}\left(g_{\geqq h}, \delta_{\geqq h}, v_{\geqq h}\right)\right. \\
& \left.+\gamma^{h} R_{4}\left(g_{\geqq h}, \delta_{\geqq h}, v_{\geqq h}\right)\right], \\
v_{h-1}= & \gamma \frac{Z_{h}}{Z_{h-1}}\left[v^{h}+v_{h} g_{h}^{2} B_{8}\left(g_{\geqq h}, \delta_{\geqq h}, v_{\geqq h}\right)+\gamma^{h} R_{5}\left(g_{\geqq h}, \delta_{\geqq h}, v_{\geqq h}\right)\right], \\
& \frac{Z_{h}}{Z_{h-1}}\left[1+g_{h, 1}^{2} \tilde{\beta}_{1}+g_{h, 2}^{2} \tilde{\beta}_{2}+g_{h}^{2} B_{9}\left(g_{\geqq h}\right)+\delta_{h} g_{h}^{2} B_{10}\left(g_{\geqq h}, \delta_{\geqq h}\right)\right. \\
& \left.+g_{h}^{2} v_{h}^{2} B_{11}\left(g_{\geqq h}, \delta_{\geqq h}, v_{\geqq h}\right)+\gamma^{h} R_{6}\left(g_{\geqq h}, \delta_{\geqq h}, v_{\geqq h}\right)\right],
\end{aligned}
$$

where by $g_{h}$ we mean generically the quartic running coupling, i.e. one of $g_{h, 1}, g_{h, 2}$, $g_{h, 4}$. We know from Sect. (4) that $B_{i}$ and $R_{l}$ are expressed by a power series in $\vec{v}_{h}$ converging to an analytic function if the running coupling $\vec{v}_{h}$ are such that $\left|\vec{v}_{h}\right| \leqq \varepsilon$. In writing Eq. (49) we make explicit the lowest order contribution in the running couplings to the power series $B_{l}$ and we use some symmetry considerations.

Using the last relation in Eq. (49) to eliminate $\frac{Z_{h}}{Z_{h-1}}$, the fact that $\gamma>1$ and the implicit function theorem it is possible to prove that the above equations are equivalent to:

$$
\begin{aligned}
\mu_{h-1} & =\mu_{h}+\hat{G}_{\mu}^{h}\left(g_{1, h}, \mu_{h} ; \ldots ; g_{1,0}, \mu_{0} ; v_{h}\right)+\gamma^{h} \hat{R}_{\mu}^{h}\left(g_{1, h}, \mu_{h} ; \ldots ; g_{1,0}, \mu_{0} ; v_{h}\right), \\
g_{1, h-1} & =g_{1, h}+\hat{G}_{1}^{h}\left(g_{1, h}, \mu_{h} ; \ldots ; g_{1,0}, \mu_{0} ; v_{h}\right)+\gamma^{h} \hat{R}_{1}^{h}\left(g_{1, h}, \mu_{h} ; \ldots ; g_{1,0}, \mu_{0} ; v_{h}\right), \\
v_{h-1} & =\gamma v_{h}+\hat{G}_{v}^{h}\left(g_{1, h}, \mu_{h} ; \ldots ; g_{1,0}, \mu_{0} ; v_{h}\right)+\gamma^{h} \hat{R}_{v}^{h}\left(g_{1, h}, \mu_{h} ; \ldots ; g_{1,0}, \mu_{0} ; v_{h}\right),
\end{aligned}
$$

where $\mu_{h}=g_{2, h}, g_{4, h}, \delta_{h}$ and $\hat{G}^{h}, \hat{R}^{h}$, are analytic for $\left|g_{1, h^{\prime}}\right|<\varepsilon,\left|\mu_{h^{\prime}}\right|<\varepsilon$, if $h^{\prime} \geqq h$ and $\left|v_{h}\right|<\varepsilon$. 
Eq. (52), (49), given any sequence of $g_{1, h}, \mu_{h}$ with $\left|g_{1, h}\right|,\left|\mu_{h}\right|<\varepsilon$, imply that there is a unique $v_{0}$, analytic in $g_{1, h}, \mu_{h}$ for $\left|g_{1, h}\right|,\left|\mu_{h}\right|<\varepsilon$, such that $\left|v_{h}\right|<\varepsilon$ and $v_{h}$ converges to 0 for $h \rightarrow-\infty$ at the rate $O\left(\gamma^{h}\right)$. The proof of the existence of $v_{0}$ is essentially a version of the unstable manifold theorem. The equation for $v_{0}$ is:

$$
v_{0}-\sum_{i=-\infty}^{0} \gamma^{i}\left[\hat{G}_{v}^{i}\left(g_{1, i}, \mu_{i} ; \ldots ; g_{1,0}, \mu_{0} ; v_{l}\right)+\gamma^{i} \hat{R}_{v}^{i}\left(g_{1, i}, \mu_{i} ; \ldots ; g_{1,0}, \mu_{0} ; v_{i}\right)\right]=0 \text {. }
$$

By Theorem 2.1 this value $v_{0}$ is obtained, given $\alpha, \lambda$, by a unique choice of $v$.

By a similar argument it is possible to choose $\delta_{0}$ (and this corresponds to fixing $\alpha)$ such that $\delta_{h} \rightarrow 0$ for $h \rightarrow-\infty$ : this choice corresponds to requiring that the Fermi velocity is fixed to 1 (see [B.G.M]) but we can avoid the work of checking the latter statement here because this choice, contrary to the choice of $v_{0}$, is not essential to control the flow of $g_{1, h}, \mu_{h}$. With the above choice of $v_{0}, \delta_{0}$ we have that

$$
\begin{aligned}
\mu_{h-1} & =\mu_{h}+\hat{G}_{\mu}^{h}\left(g_{1, h}, \mu_{h} ; \ldots ; g_{1,0}, \mu_{0} ; 0\right)+\gamma^{h} \hat{R}_{\mu}^{h}\left(g_{1, h}, \mu_{h} ; \ldots ; g_{1,0}, \mu_{0} ; v_{h}\right), \\
g_{1, h-1} & =g_{1, h}+\hat{G}_{1}^{h}\left(g_{1, h}, \mu_{h} ; \ldots ; g_{1,0}, \mu_{0} ; 0\right)+\gamma^{h} \hat{R}_{1}^{h}\left(g_{1, h}, \mu_{h} ; \ldots ; g_{1,0}, \mu_{0} ; v_{h}\right), \\
v_{h-1} & =\gamma v_{h}+\hat{G}_{v}^{h}\left(g_{1, h}, \mu_{h} ; \ldots ; g_{1,0}, \mu_{0} ; 0\right)+\gamma^{h} \hat{R}_{v}^{h}\left(g_{1, h}, \mu_{h} ; \ldots ; g_{1,0}, \mu_{0} ; v_{h}\right) .
\end{aligned}
$$

Remembering that $\hat{G}_{1}^{h}\left(g_{1, h}, \mu_{h} ; \ldots ; g_{1,0}, \mu_{0} ; 0\right)=g_{1, h}^{2}\left(-2 \beta+\bar{G}_{1}^{h}\left(g_{1, h}, \mu_{h} ; \ldots ; g_{1,0}\right.\right.$, $\left.\mu_{0} ; 0\right)$, with $\bar{G}_{1}^{h}$ analytic as a function of its argument, it is clear that, for any sequence of $\left|\mu_{h}\right|<\varepsilon$ and any complex $g_{1,0}$, such that $\left|g_{1,0}-\varepsilon / 2\right|<\varepsilon / 2$, then $\mid g_{1, h}-$ $\varepsilon / 2 \mid \leqq \varepsilon / 2$ and $g_{1, h} \rightarrow 0$ for $h \rightarrow-\infty$ as $O\left(\frac{1}{|h|}\right)$.

Remark. Chosen $v$ as above and $\lambda$ complex such that $|\lambda-\tilde{\varepsilon} / 2|<\tilde{\varepsilon} / 2$, where $\tilde{\varepsilon}$ is chosen so that $\left|v_{0}\right|,\left|\mu_{0}\right|<\varepsilon$ and $\left|g_{1,0}-\varepsilon / 2\right| \leqq \varepsilon / 2$, if it happens that $\left|\mu_{h^{\prime}}\right|<\varepsilon$ for $h^{\prime} \geqq h$, then $\vec{v}_{h^{\prime}}$ for $h^{\prime} \geqq h-1$ is analytic as a function of $\lambda$ in $|\lambda-\tilde{\varepsilon} / 2|<\tilde{\varepsilon} / 2$ and $v_{h}, g_{1, h} \rightarrow 0$ for $h \rightarrow \infty$.

We want to show that $\left|\mu_{h}\right|<\varepsilon$ for all $h$ if $\left|\lambda-\frac{\tilde{\varepsilon}}{2}\right|<\frac{\tilde{\varepsilon}}{2}$. We define the function $\lim _{h \rightarrow-\infty} \lim _{T, L \rightarrow \infty} \hat{G}_{i}^{h}(v ; \ldots ; v ; 0)=G_{i}(v)$, where $i=\mu, v, 1$ and $v=g_{1}, \mu$. We prove that:

$$
G_{\mu}(0, \mu)=0 .
$$

Equation (55) can be proved by comparing the beta function of our system with the Beta function of the Mattis model. The Mattis model describes two spinning fermions with linear dispersion relation. The Hamiltonian is:

$$
\begin{aligned}
T_{0}^{\prime}+H_{I}^{\prime}= & \sum_{\vec{\omega}, \sigma} \int d \vec{x}: \psi_{\vec{x}, \vec{\omega}, \sigma}^{+}(i \vec{\omega} \vec{\partial}) \psi_{\vec{x}, \vec{\omega}, \sigma}^{-}:+\alpha \sum_{\vec{\omega}, \sigma} \int d \vec{x}: \psi_{\vec{x}, \vec{\omega}, \sigma}^{+}(i \vec{\omega} \vec{\partial}) \psi_{\vec{x}, \vec{\omega}, \sigma}^{-}: \\
& +\sum_{\vec{\omega}, \sigma, \sigma^{\prime}} \int d \vec{x} d \vec{y} \lambda g_{4}(\vec{x}-\vec{y}):\left(\psi_{\vec{x}, \vec{\omega}, \sigma}^{+} \psi_{\vec{x}, \vec{\omega}, \sigma}^{-}\right)::\left(\psi_{\vec{y}, \vec{\omega}, \sigma^{\prime}}^{+} \psi_{\vec{y}, \vec{\omega}, \sigma^{\prime}}^{-}\right): \\
& +\sum_{\vec{\omega}, \sigma, \sigma^{\prime}} \int d \vec{x} d \vec{y} \lambda g_{2}(\vec{x}-\vec{y}):\left(\psi_{\vec{x}, \vec{\omega}, \sigma}^{+} \psi_{\vec{x}, \vec{\omega}, \sigma}^{-}\right)::\left(\psi_{\vec{y},-\vec{\omega}, \sigma^{\prime}}^{+} \psi_{\vec{y},-\vec{\omega}, \sigma^{\prime}}^{-}\right):
\end{aligned}
$$

where :: denotes the Wick ordering respect to the free vacuum and $\psi_{\vec{x}, \vec{\omega}, s}^{ \pm}$are creation or annihilation operators of $\vec{\omega}$-fermions. Note that, contrary to the model with hamiltonian Eq. (1), the Mattis model hamiltonian is written directly in terms of quasiparticles. We can introduce a family of Grassmannian variables $\psi_{x, \vec{\omega}, \sigma}^{\varepsilon}$ and study the 
Mattis model by a renormalization group analysis similar to the one discussed so far. The ultraviolet part of the theory is rather delicate, due to the linear dispersion relation of the propagator of the Mattis model, but it has been discussed in [G.Sc.] (only for the Luttinger model, but the same considerations trivially hold also for the Mattis model).

The discussion of the infrared part is made by repeating the arguments in Sect. 2 - Sect. 4 with $U(\vec{x})=0$. The infrared integration is written as $\prod_{\varepsilon, \sigma, \vec{\omega}= \pm 1}$ $\prod_{h=-\infty}^{0} P\left(d \psi_{k, \tilde{\omega}, \sigma}^{\varepsilon, h}\right)$, where the covariance of $\psi_{k, \hat{\omega}, \sigma}^{\varepsilon, h}$ is

$$
g_{\overrightarrow{\bar{\omega}}, k^{\prime}, M}^{\geqq h}=\frac{\sum_{h^{\prime}=h}^{0} f\left(\gamma^{-2 h^{\prime}}\left(k_{0}^{2}+\vec{k}^{2}\right)\right.}{-i k_{0}+\vec{\omega} \vec{k}^{\prime}} .
$$

The effective potential is given by Eq. (24) with $g_{1, h}=g_{3, h}=0$ and $v_{h}=0$ by the symmetry of the interaction and for the parity of the Mattis model propagator.

The Beta function is given by $\mu_{h-1}=\mu_{h}+B_{\mu, M}^{h}\left(\mu_{h} ; \ldots ; \mu_{0}\right)+\gamma^{h} \hat{R}_{\mu}^{h}\left(\mu_{h} ; \ldots ; \underline{\mu}_{0}\right)$. The crucial point is that

$$
B_{\mu, M}^{h}\left(\mu_{h} ; \ldots ; \mu_{0}\right)=G_{\mu}^{h}\left(\mu_{h}, 0 ; \ldots ; \mu_{0}, 0 ; 0\right) .
$$

This essentially follows from the fact that the propagators $g_{\vec{\omega}}^{h}(x)$ of our model differ from the Mattis one only for terms of order $\gamma^{h}$ (see Lemma 2.1) and from the fact that in our model $v_{h}=O\left(\gamma^{h}\right)$. The analysis in the preceding section shows that the Beta function is analytic as a function of its argument in a circle with radius independent from $\beta, L$ (see Th. 4.1); this implies that the limit of the Beta function as $\beta \rightarrow \infty$ is an analytic function of its argument in the same domain. We call from now on $\mu_{h}^{L}$ the running coupling in the theory with finite volume (but not temporal cut-off) and $\lim _{L \rightarrow \infty} \mu_{h}^{L}=\mu_{h}$. If $L_{h}=p_{0}^{-1} \gamma^{-h}$ the following lemma, analogous to Lemma 2 in [B.G.P.S.], holds:

Lemma 5.1. If $\mu_{h^{\prime}}$ is defined and $\left|\mu_{h^{\prime}}\right| \leqq \varepsilon_{1} \leqq \varepsilon / 2$, for $h^{\prime} \geqq h$, then also $\mu_{h^{\prime}}^{L_{h}}$ is defined for $h^{\prime} \geqq h$ and

$$
\left|\mu_{h^{\prime}}^{L_{h}}-\mu_{h^{\prime}}\right| \leqq c_{1} \varepsilon_{1}^{3 / 2} \gamma^{h-h^{\prime}} \quad h^{\prime} \geqq h
$$

for some constant $c_{1}$.

Proof. We proceed inductively noting that Eq. (58) holds for $h^{\prime}=0$ and proving that Eq. (58) holds for $h^{\prime}-1$ if it holds for the couplings with frequency greater than or equal to $h^{\prime}$. We write

$$
\mu_{h^{\prime}-1}^{L_{h}}-\mu_{h^{\prime}-1}=\mu_{h^{\prime}}^{L_{h}}-\mu_{h^{\prime}}+\beta_{\mu, M}^{h^{\prime}, L_{h}}\left(\mu_{h^{\prime}}^{L_{h}}, \ldots, \mu_{0}^{L_{h}}\right)-\beta_{\mu, M}^{h^{\prime}}\left(\mu_{h^{\prime}}, \ldots, \mu_{0}\right),
$$

where $\beta_{\mu, M}^{h^{\prime}, L_{h}}$ and $\beta_{\mu, M}^{h^{\prime}}$ are the Beta functions for the Mattis model with finite or infinite volume. It is convenient to write the second difference in Eq. (59) as

$$
\left[\beta_{\mu, M}^{h^{\prime}, L_{h}}\left(\mu_{h^{\prime}}^{L_{h}}, \ldots, \mu_{0}^{L_{h}}\right)-\beta_{\mu, M}^{h^{\prime}, L_{h}}\left(\mu_{h^{\prime}}, \ldots, \mu_{0}\right)\right]+\left[\beta_{\mu, M}^{h^{\prime}, L_{h}}\left(\mu_{h^{\prime}}, \ldots, \mu_{0}\right)-\beta_{\mu, M}^{h^{\prime}}\left(\mu_{h^{\prime}}, \ldots, \mu_{0}\right)\right] .
$$

The first term can be bounded, proceeding as in Sect. 4 and using the inductive assumption, by $c_{2} \varepsilon_{1}^{5 / 2} \gamma^{h-h^{\prime}}$. In order to estimate the second difference in Eq. (60) 
we remember that $\beta_{\mu, M}^{h^{\prime}}\left(\mu_{h^{\prime}}, \ldots, \mu_{0}\right)$ can be written, by Eq. (42), (43), as a sum over trees $\tau$; to each tree is associated a product of terms which can be written as the integral of products of propagators whose arguments $\xi_{l}-\eta_{l}$ form an anchored tree graph $T$ times a determinant; all except one of the points belonging to the anchored tree graph are integrated. We perform a change of variables $\xi_{l}-\eta_{l}=y_{l}$ as in Eq. (46) and we write

$$
\beta_{\mu, M}^{h^{\prime}}\left(\mu_{h^{\prime}}, \ldots, \mu_{0}\right)=\hat{\beta}_{\mu, M}^{h^{\prime}}\left(\mu_{h^{\prime}}, \ldots, \mu_{0}\right)+\tilde{\beta}_{\mu, M}^{h^{\prime}}\left(\mu_{h^{\prime}}, \ldots, \mu_{0}\right)
$$

where $\hat{\beta}_{\mu, M}^{h^{\prime}}$ contains terms integrated in $\left|\vec{y}_{l}\right| \leqq L / 2$ for each $y_{l}$ belonging to the spanning tree. All the terms contained in $\tilde{\beta}_{\mu, M}^{h^{\prime}}$ contain at least an integral with domain $\left|\vec{y}_{l}\right|>L / 2$ so that, proceeding as in Sect. 4 and remembering Eq. (46), it follows that $\tilde{\beta}_{\mu, M}^{h^{\prime}}$ is bounded by $c_{4} \varepsilon_{1}^{2} \gamma^{h-h^{\prime}}$.

It remains to bound $\beta_{\mu, M}^{h^{\prime}, L_{h}}\left(\mu_{h^{\prime}}, \ldots, \mu_{0}\right)-\hat{\beta}_{\mu, M}^{h^{\prime}}\left(\mu_{h^{\prime}}, \ldots, \mu_{0}\right)$; this can made by noting that this term can be written as a sum over trees similar to that one in Sect. 4 for $\beta^{h^{\prime}, L}$, with the integrals over the arguments of the spanning tree $T$ with domain $\left|\vec{y}_{l}\right| \leqq L / 2$ : the only difference is that at least one of the propagators of the spanning tree $g^{h^{\prime \prime}, L}\left(y_{l}\right), h^{\prime \prime}>h^{\prime}$ is replaced by $g^{h^{\prime \prime}}\left(y_{l}\right)-g^{h^{\prime \prime}, L_{h}}\left(y_{l}\right)$, or at least one of the determinants is replaced by $\operatorname{det} G^{T}-\operatorname{det} G^{T, L_{h}}$. Noting that the number of possible substitutions is bounded by $C^{n}$, if $\tau \in \tau_{n}$, and that

$$
\begin{gathered}
\left|g^{h^{\prime}}(y)-g^{h^{\prime}, L_{h}}(y)\right| \leqq \frac{\gamma^{-h^{\prime}}}{L_{h}} \frac{C_{N}}{1+\gamma^{h^{\prime} N}|y|^{N}},|\vec{y}| \leqq \frac{L}{2} \\
\left|\operatorname{det} G^{T}-\operatorname{det} G^{T, L_{h}}\right| \leqq \frac{C^{|P|}}{L_{h}} \gamma^{h|P| / 2}
\end{gathered}
$$

if $|P|$ is the number of the fields in $G$ and proceeding as in Sect. 4 we find that this term is bounded by $c_{3} \varepsilon_{1}^{2} \gamma^{h-h^{\prime}}$. Finally we can write

$$
\begin{aligned}
\left|\mu_{h^{\prime}-1}^{L_{h}}-\mu_{h^{\prime}-1}\right| & \leqq c_{1} \varepsilon_{1}^{3 / 2} \gamma^{h-h^{\prime}}+c_{2} \varepsilon_{1}^{5 / 2} \gamma^{h-h^{\prime}}+c_{3} \varepsilon_{1}^{2} \gamma^{h-h^{\prime}}+c_{4} \varepsilon_{1}^{2} \gamma^{h-h^{\prime}} \\
& \leqq c_{1} \varepsilon_{1}^{3 / 2} \gamma^{h-h^{\prime}+1}
\end{aligned}
$$

where the first term comes from the first difference in Eq. (59) and the last three from the second difference in Eq. (60). The above inequality is always verified if $\varepsilon_{1}$ is chosen suitable small.

Let us remark that the effective potential at scale $h$ can be also obtained by a one step integration by the relation

$$
\left.e^{V^{h}(\psi \leqq h}\right)=\frac{\mathscr{N}_{0}}{\mathscr{N}} \int P\left(d \psi^{>h}\right) e^{-V^{0}\left(\psi^{\leqq 0}\right)} .
$$

$V^{h}\left(\psi^{\leqq}\right)$is given by a series over one step Feynman graphs similar to those ones of Sect. 4 except that to each internal line is associated the propagator $g_{\vec{\omega}}^{>h}(k)=\sum_{k=h+1}^{0} g_{\vec{\omega}}^{h}(k)$ and to each vertex one of the terms in $V^{0}$ Eq. (17), (18). The expansion is well defined if $|\lambda| \leqq \frac{\text { const }}{L}$, which is not $O(1)$, and hence very 
small as $L \rightarrow \infty$. By the definition of the localization in Sect. (4) it follows that

$$
\begin{gathered}
Z_{h}^{L} \gamma^{h} v_{h}=\frac{1}{4} \sum_{j=1}^{2} V_{2}^{h}\left(0,(-1)^{j} \frac{\pi}{L} ; \vec{\omega}\right)=0 ; Z_{h}^{L}-1=\left.\frac{i \partial}{\partial k_{0}} \sum_{i=1}^{2} V_{2}^{h}\left(k_{0},(-1)^{2} \frac{\pi}{L} ; \vec{\omega}\right)\right|_{k_{0}=0}, \\
Z_{h}^{L}-1+Z_{h}^{L} \delta_{h}^{L}=\frac{\vec{\omega} L}{2 \pi}\left(V_{2}^{h}\left(0, \frac{\pi}{L} ; \vec{\omega}\right)-V_{2}^{h}\left(0,-\frac{\pi}{L} ; \vec{\omega}\right)\right), \\
\left(Z_{h}^{L}\right)^{2} g_{2, h}^{L}=\frac{1}{2} \sum_{k} V_{4}^{h}\left(\left(0,(-1)^{k} \frac{\pi}{L}\right) ;\left(0,(-1)^{k} \frac{\pi}{L}\right) ;\left(0,(-1) \frac{\pi}{L}\right) ;\left(0,(-1)^{k} \frac{\pi}{L}\right) ; \underline{\vec{\omega}}_{1}\right), \\
\left(Z_{h}^{L}\right)^{2} g_{4, h}^{L}=\frac{1}{2} \sum_{k} V_{4}^{h}\left(\left(0,(-1)^{k} \frac{\pi}{L}\right) ;\left(0,(-1)^{k} \frac{\pi}{L}\right) ;\left(0,(-1) \frac{\pi}{L}\right) ;\left(0,(-1)^{k} \frac{\pi}{L}\right) ; \underline{\vec{\omega}}_{2}\right),
\end{gathered}
$$

where $V_{2}^{h}\left(k^{\prime} ; \vec{\omega}\right)$ is given by the sum over all the one step graphs with two external lines with momentum $\overrightarrow{k^{\prime}}+\vec{\omega} p_{F}$ and quasi-particle index $\vec{\omega}$ and $V_{4}^{h}\left(k_{1}^{\prime}, k_{2}^{\prime}, k_{3}^{\prime}, k_{4}^{\prime} ; \underline{\omega}_{i}\right)$ is given by the sum over the one step Feynman graphs with four external lines with momentum $k_{\imath}^{\prime}+\vec{\omega}_{i} p_{F}$ and quasi-particle index $\vec{\omega}_{i}, i=1,2,3,4$ and $\underline{\omega}_{1}=$ $(1,-1,-1,1), \underline{\vec{\omega}}_{2}=(1,1,1,1)$. Of course the one step expansion for $V_{2}\left(k^{\prime} ; \vec{\omega}\right)^{h}$ and $V_{4, i}^{h}\left(k_{1}^{\prime}, k_{2}^{\prime}, k_{3}^{\prime}, k_{4}^{\prime} ; \underline{\underline{\vec{\omega}}}\right)$ is convergent only if $|\lambda| \leqq \frac{\text { const }}{L}$. If $L \equiv L_{h-n}$ and $n>2$ it follows from the compact support properties of $g_{\vec{\omega}}{ }^{h}(k)$ that $g_{\vec{\omega}}{ }^{h}(k) \equiv 0$ in a small domain around the point $(\pi / L, 0)$ so that the one step graphs contributing to $\mu_{h}^{L_{h-n}}$ or $Z_{h}^{L_{h-n}}$ are only the irreducible ones, defined as graphs which cannot be splitted into two parts by cutting a single internal line. We call $\sum^{h}(k ; \vec{\omega})$ and $\Gamma_{l}^{h}\left(k_{1}, k_{2}, k_{3}, k_{4} ; \underline{\vec{\omega}}\right)$ the sum over the irreducible one step graphs contributing to $V_{2}^{h}\left(k^{\prime} ; \vec{\omega}\right)$ and $V_{4, i}^{h}\left(k_{1}^{\prime}, k_{2}^{\prime}, k_{3}^{\prime}, k_{4}^{\prime} ; \underline{\underline{\omega}}\right)$. Then Eq. (62) can be written, if $L \equiv L_{h-n}$ and $n>2$, replacing $V_{2}^{h}$ and $V_{4}^{h}$ by $\sum^{h}$ and $\Gamma^{h}$.

By definition $\sum^{h}(k ; \vec{\omega})$ and $\Gamma_{i}^{h}\left(k_{1}, k_{2}, k_{3}, k_{4} ; \underline{\vec{\omega}}\right)$ are simply related to the two point or truncated four point Schwinger function with infrared cut-off at scale $h$ :

$$
\begin{gathered}
S^{>h, L}\left(k^{\prime}, \vec{\omega}\right)=\frac{1}{-i k_{0}+\vec{\omega} \overrightarrow{k^{\prime}}+\sum^{h}\left(k^{\prime}\right)}, \\
S_{4}^{>h, T, L}\left(k_{1}^{\prime}, \vec{\omega}_{1},+, \sigma ; k_{2}^{\prime}, \vec{\omega}_{2},+, \sigma^{\prime} ; k_{3}^{\prime}, \vec{\omega}_{3},-, \sigma^{\prime} ; k_{4}^{\prime}, \vec{\omega}_{4},-, \sigma\right)= \\
S^{>h, L}\left(k_{1}^{\prime}, \vec{\omega}_{1}\right) S^{>h, L}\left(k_{2}^{\prime}, \vec{\omega}_{2}\right) S^{>h, L}\left(k_{3}^{\prime}, \vec{\omega}_{3}\right) S^{>h, L}\left(k_{4}^{\prime}, \vec{\omega}_{4}\right) \Gamma^{h}\left(k_{1}^{\prime}, k_{2}^{\prime}, k_{3}^{\prime}, k_{4}^{\prime} ; \underline{\vec{\omega}}\right) .
\end{gathered}
$$

The above equations can be proven as an identity between graph at any order.

Equations (62),(63), (64) by substitution and some algebra implies that if $L \equiv L_{h-n}$ and $n>2$ :

$$
\begin{gathered}
\frac{L}{\pi} \frac{1}{Z_{h}^{L}\left(1+\delta_{h}^{L}\right)}=S^{>h, L}(0, \pi / L ; \vec{\omega}) \quad ; \quad \frac{L^{2}}{\pi^{2}} \frac{1}{Z_{h}^{L}\left(1+\delta_{h}^{L}\right)^{2}}=\frac{\partial S^{>h, L}(0, \pi / L ; \vec{\omega})}{\partial k_{0}}, \\
\left(\frac{L}{\pi\left(1+\delta_{h}^{L}\right)}\right)^{4} \frac{g_{2, h}^{L}}{\left(Z_{h}^{L}\right)^{2}}=\sum_{l=1}^{2} S^{>h, T, L}\left(0,(-1)^{l} \pi / L ; 0,(-1)^{i} \pi / L ; 0,\right. \\
\left.(-1)^{i} \pi / L ; 0,(-1)^{l} \pi / L ; \underline{\vec{\omega}}_{1}\right), \\
\left(\frac{L}{\pi\left(1+\delta_{h}^{L}\right)}\right)^{4} \frac{g_{4, h}^{L}}{\left(Z_{h}^{L}\right)^{2}}=\sum_{i=1}^{2} S^{>h, T, L}\left(0,(-1)^{l} \pi / L ; 0,(-1)^{i} \pi / L ; 0,\right. \\
\left.(-1)^{i} \pi / L ; 0,(-1)^{l} \pi / L ; \underline{\vec{\omega}}_{2}\right),
\end{gathered}
$$


Remark. The above identity is proved by a one step integration, so is proved in the region $|\lambda| \leqq c o n s t / L$, where the one step expansion for the effective potential and the Schwinger function is analytic. However the series for the effective potential and Schwinger functions at scale $h$ are analytic in $\lambda$ if the running coupling constants $\mu_{h^{\prime}}, h^{\prime}>h$ are such that $\left|\mu_{h^{\prime}}\right|<\varepsilon$, see Sect. 4 and below, so that in this case Eq. (65), (66) holds also in the domain $\left|\mu_{h^{\prime}}\right|<\varepsilon$.

$\mu_{\infty}^{L}, Z_{\infty}^{L}$, if $S^{>-\infty, L} \equiv S^{L}$ are given by Eq. (65), if $S^{>-\infty, T, L} \equiv S^{T, L}$ replaces $S^{>h, T, L}$.

Using the exact solution of the Mattis model [M.] and the explicit evaluation of its Schwinger function [Ma1] it is possible to prove the following lemma (analogous to Lemma 3 of [B.G.P.S.] but not explicitly proved there):

Lemma 5.2. In the Mattis model there exists an $\varepsilon$ such that, if $|\lambda| \leqq \varepsilon$, then $\mu_{\infty}^{L}$ is bounded and $\left|\mu_{\infty}^{L}\right| \leqq$ const $\lambda$ uniformly in $L$; moreover $Z_{\infty}^{L}=A_{L}(\hat{\lambda}) L^{2 \eta(\hat{\lambda})}$ with $A_{L}(\lambda)$ and $\eta(\hat{\lambda})$ bounded in $\lambda$ for $|\lambda| \leqq \varepsilon$ and $O\left(\hat{\lambda}^{2}\right)$.

Proof. If $\varepsilon_{\rho}(p)=\operatorname{sech} \phi(\vec{p})_{\rho}=\sqrt{\left(1+\alpha+\frac{\lambda \hat{g}_{4}(\vec{p})}{\pi}\right)^{2}+\left(\frac{\lambda \hat{g}_{2}(\vec{p})}{\pi}\right)^{2}}$ in [Ma1] it is shown that

$$
\begin{gathered}
S^{L}(x, \vec{\omega})=\bar{S}_{0}^{L}(x, \vec{\omega}) e^{-Q_{\rho}^{L}(x)} \\
\bar{S}_{0}^{L}(x, \vec{\omega})=\frac{\theta\left(x_{0}\right)}{L} \frac{e^{-\frac{c}{L}\left(x_{0}+l \vec{x}\right)}}{1-e^{-\frac{2 \pi}{L}\left(x_{0}+l \vec{x}\right)}}-\frac{\theta\left(-x_{0}\right)}{L} \frac{e^{-\frac{c}{L}\left(\left|x_{0}\right|-i \vec{x}\right)}}{1-e^{-\frac{2 \pi}{L}\left(\left|x_{0}\right|+i \vec{x}\right)}}=e^{-\frac{c}{L}\left|x_{0}\right|} \tilde{S}(x ; \vec{\omega}), \\
Q_{\rho}^{L}=\frac{\pi}{L} \sum_{\vec{p}>0} \frac{S_{\rho}(\vec{p})^{2}}{\vec{p}}\left(1-e^{-\vec{p}|t| \varepsilon_{\rho}(\vec{p})} \cos \vec{p} \vec{x}\right)-\frac{\pi}{L} \sum_{\vec{p}>0} \frac{\cos \vec{p} \vec{x}}{\vec{p}}\left(e^{-\vec{p}|t|}-e^{-\vec{p}|t| \varepsilon_{\rho}(\vec{p})}\right) \\
-\frac{\pi}{L} \sum_{\vec{p}>0} \frac{\sin \vec{p} \vec{x}}{\vec{p}}\left(e^{-\vec{p}|t|}-e^{-\vec{p}|t| \varepsilon_{\rho}(\vec{p})}\right),
\end{gathered}
$$

where $c=\pi+\lambda g_{4}(0)>0$ which is the solubility condition of the Mattis model, $S_{\rho}(p)=\sinh \phi(p)_{\rho}$ and $\hat{g}_{i}(\vec{p})$ is the Fourier transform of $g_{l}(\vec{r})$. In the limit $L \rightarrow \infty$ the asymptotic behaviour of the two point Schwinger function is [Ma1]:

$$
\frac{1}{i \vec{\omega} \vec{x}+\varepsilon(o) t} \frac{1}{\left(\vec{x}^{2}+\varepsilon(0)^{2} t^{2}\right)^{\eta}}\left(A(\phi)+A_{1}(\phi, x)\right),
$$

where $A_{1}(\phi, x)$ is bounded near $\hat{g}_{2}(\vec{p})=\hat{g}_{4}(\vec{p})=0$ and $\eta=\frac{1}{2}\left(1-\frac{\lambda \hat{g}_{2}(0)^{2}}{\left.\left(2 \pi(1+\alpha)+\lambda \hat{g}_{4}(0)\right)^{2}\right)}\right)^{-1 / 2}$ $-1 / 2 . \varepsilon(0)$ is the Fermi velocity. It is possible to choose $\alpha$ as an analytic function of $\lambda$ so that $\varepsilon(0)=1$.

From Eq. (65), (66) it is easy to see that $Z_{\infty}^{L}=L^{2 \eta} \frac{I_{2}^{L}}{\left(I_{1}^{L}\right)^{2}} \delta_{\infty}^{L}=-1+\frac{I_{1}^{L}}{\pi I_{2}^{L}}$, where

$$
\begin{aligned}
& S^{L}(0, \pi / L ; 1)=\frac{L}{L^{2 \eta}} \int_{0}^{\infty} d x_{0} \int_{-\frac{1}{2}}^{\frac{1}{2}} d \vec{x} e^{-c x_{0}}\left(L^{1+2 \eta} \tilde{S}^{L}(L x) e^{-Q_{\rho}^{L}(x L)}\right) e^{i \pi \vec{x}}=\frac{L}{L^{2 \eta}} I_{1}^{L}, \\
& \partial_{k_{0}} S^{L}(0, \pi / L ; \vec{\omega})=\frac{L^{2}}{L^{2 \eta}} \int_{0}^{\infty} d x_{0} \int_{-\frac{1}{2}}^{\frac{1}{2}} d \vec{x} e^{-c x_{0}} x_{0}\left(L^{1+2 \eta} \tilde{S}^{L}(L x) e^{-Q_{\rho}^{L}(x L)}\right) e^{i \pi \vec{x}}=\frac{L^{2}}{L^{2 \eta}} I_{2}^{L} .
\end{aligned}
$$


From Eq. (69) it follows that $I_{1}^{L}, I_{2}^{L}$ has a limit for $L \rightarrow \infty$.

We study now the four points Schwinger function. We limit ourselves to $S^{L}\left(x_{1},+, \sigma ; x_{2},+, \sigma^{\prime} ; x_{3},-, \sigma^{\prime} ; x_{4},-, \sigma\right)$ but similar considerations could be made also for $S^{L}\left(x_{1},+, \sigma ; x_{2},+,-\sigma ; x_{3},-,-\sigma ; x_{4},-, \sigma\right)$. In [Ma1] it is shown that

$$
S^{T, L}\left(x_{1},+, \sigma ; x_{2},+, \sigma^{\prime} ; x_{3},-, \sigma^{\prime} ; x_{4},-, \sigma\right)=S^{L}\left(x_{1}-x_{4} ; 1\right) S^{L}\left(x_{2}-x_{3} ;-1\right)\left(e^{A}-1\right),
$$

where $A=F\left(x_{1}-x_{3}\right)+F\left(x_{2}-x_{4}\right)-F\left(x_{1}-x_{2}\right)-F\left(x_{3}-x_{4}\right)$ and $F(x)=\frac{2 \pi}{L} \sum_{\vec{p}}$ $\frac{s_{\rho}(\vec{p}) c_{\rho}(\vec{p})}{\vec{p}}\left(1-e^{-\vec{p}\left|x_{0}\right| \varepsilon_{\rho}(\vec{p})} \cos \vec{p} \vec{x}\right)$ with $c_{\rho}(p) \tau \cosh \phi(\vec{p})_{c}$. Performing the change of variables $u=x_{1}-x_{4}, v=x_{2}-x_{3}, z=x_{1}-x_{3}$ we can write

$$
\begin{aligned}
\frac{g_{2, \infty}^{L}\left(I_{2}^{L}\right)^{2} L^{4}}{L^{4 \eta}}= & \frac{L^{4}}{L^{4 \eta}} \int_{0}^{\infty} d u_{0} \int_{-1}^{1} d \vec{u} e^{-c u_{0}}\left(L^{1+2 \eta} \tilde{S}^{L}(L u) e^{-Q_{\rho}^{L}(u L)}\right. \\
& \times \int_{0}^{\infty} d v_{0} \int_{-1}^{1} d \vec{v} e^{-c v_{0}}\left(L^{1+2 \eta} \tilde{S}^{L}(L v) e^{-Q_{\rho}^{L}(v L)}\right) \\
& \left.\times \int_{0}^{\infty} d z_{0} \int_{-1}^{1} d \vec{z}(\cos \pi \vec{u} \cos \pi \vec{v}-\sin \pi \vec{u} \sin \pi \vec{v})\left(e^{A \rho(u L, v L, z L)}-1\right)\right\}
\end{aligned}
$$

It is easy to see that the expression between curly brackets of the r.h.s. of Eq. (71) has a limit for $L \rightarrow \infty$. This is made by using the asymptotic expressions of $S^{L}(x, \vec{\omega})$, Eq. (69), and of $F(x)$ (i.e. $s(0) c(0) \log \left|x_{0}^{2}+\varepsilon_{\rho}^{2}(0) \vec{x}^{2}\right|$ ) and by dividing the integration domain of the integral in the r.h.s. of Eq. (71) in several regions:

$$
\begin{gathered}
D_{1}=\left\{u_{0}, v_{0}, z_{0} \leqq 1\right\}, \quad D_{2}=\left\{u_{0}, v_{0} \geqq 1, z_{0} \leqq 1\right\}, \quad D_{3}=\left\{u_{0}, v_{0} \leqq 1, z_{0} \geqq 1\right\}, \\
D_{4}=\left\{u_{0} \leqq 1, z_{0}, v_{0} \geqq 1\right\}, \quad D_{5}=\left\{v_{0} \leqq 1, z_{0}, u_{0} \geqq 1\right\}, \quad D_{6}=\left\{u_{0}, v_{0}, z_{0} \geqq 1\right\} .
\end{gathered}
$$

In each region the infrared divergences are integrable. In $D_{2}$ the integration over $u_{0}, v_{0}$ is controlled by the exponential factors; in $D_{3}$ one has to use that $\left(e^{A}-1\right) \simeq_{z_{0} \rightarrow \infty} \frac{f\left(u_{0}, v_{0}, \vec{u}, \vec{v}, \vec{z}\right)}{z_{0}^{2}}$, where $f\left(u_{0}, v_{0}, \vec{u}, \vec{v}, \vec{z}\right)$ is a polynomial of second order in the variables $u_{0}, v_{0}, \vec{u}, \vec{v}, \vec{z}$; in $D_{4}$ it is convenient to split the integration domain in two regions, one with $\left|z_{0}-v_{0}\right| \leqq 1$ and the other with $\left|z_{0}-v_{0}\right| \geqq 1$. In the first of these regions one can perform a change of variables $u_{0}, v_{0}, z_{0} \rightarrow y_{0}=z_{0}-v_{0}, u_{0}, v_{0}$ and the integral is of course divergence free as $y_{0}, u_{o} \leqq 1$, while $v_{0}$ is controlled by the exponential term; in the second region one can use that $\left(e^{A}-1\right) \simeq_{x_{0} \rightarrow \infty}$ $\frac{f_{1}\left(u_{0}, v_{0}, \vec{u}, \vec{v}, \vec{z}\right)}{\left(z_{0}-v_{0}\right)^{2}}+\frac{f_{2}\left(u_{0}, v_{0}, \vec{u}, \vec{v}, \vec{z}\right)}{z_{0}\left(z_{0}-v_{0}\right)}$. Similar considerations hold for $D_{5}$ and $D_{6}$.

Suppose now that $\mu_{h}$, although start arbitrary small, can reach $O(\varepsilon / 2)$ at $h_{0}$; then $v_{h_{0}}$ is "close" to $v_{h_{0}}^{L_{h_{0}}}$, by the considerations above Eq. (58); but, by the consideration at the end of Sect. $3, v_{h_{0}}^{L_{h_{0}}}=v_{-\infty}^{L_{h_{0}}}$, so we can conclude that $v_{-\infty}^{L_{h_{0}}}=O(\varepsilon / 2)$. But this is in contradiction with the fact that, by the exact solution $v_{-\infty}^{L_{h_{0}}}=O(\lambda)$ (Lemma 5.1 ); this essentially proves that:

Lemma 5.3. There exists an $\varepsilon$ such that, if $|\lambda| \leqq \varepsilon$ then for any $h \leqq 0 \mu_{h}$ is analytic in $\lambda$ and $\left|\mu_{h}\right|<$ const $\cdot \lambda$ uniformly in $L ;$ moreover $Z_{h}=A_{L}(\bar{\lambda}) \gamma^{-h 2 \eta(\lambda)}$ with $A_{L}(\lambda), \eta(\lambda)$ analytic in $\lambda$ in $|\lambda|<\varepsilon$ and $O\left(\lambda^{2}\right)$. 
Proof. Let us suppose that, given $\varepsilon_{1} \leqq \varepsilon / 2$, there exists $h_{0}>-\infty$ such that

$$
\left|\mu_{h}\right| \leqq \varepsilon_{1} / 2<\left|\mu_{h_{0}}\right|<\varepsilon_{1}
$$

for $h \geqq h_{0}$. We start with a small $\mu$, say $|\mu| \leqq \varepsilon_{1} / 4$ and, from Lemma 5.1 we have that it is possible to fix $n>2$ so that, if $h^{\prime} \geqq h_{0}\left|\mu_{h^{\prime}}-\mu_{h^{\prime}}^{L_{h_{0}-n}}\right| \leqq c_{1} \varepsilon^{3 / 2} \gamma^{-n} \leqq \varepsilon_{1} / 8$ (for instance). Note that, if $\left|\mu_{h^{\prime}}\right| \leqq \varepsilon_{1} \leqq \varepsilon, h^{\prime} \geqq h$ the bounds in Sect. 4 imply that

$$
\left|\mu_{h^{\prime}}^{L}-\mu_{h^{\prime}+1}^{L}\right| \leqq \varepsilon_{1}^{2},
$$

and this equation imply that $\left|\mu_{h_{0}}^{L_{h_{0}-n}}-\mu_{h_{0}-n}^{L_{h_{0}-n}}\right| \leqq 2 b \varepsilon_{1}^{2} n$ and the factor 2 takes into account the small growth of $\mu_{h^{\prime}}^{L_{h_{0}-n}}$ for $h<h_{0}$. But by Eq. (36) it holds that $\mu_{h_{0}-n}^{L_{h_{0}-n}}=\mu_{\infty}^{L_{h_{0}-n}}$ and, $\mu_{\infty}^{L_{h_{0}-n}}$, defined by Eq. (65), (66) with $h=-\infty$, is close to $\mu$ by $c_{2} \varepsilon_{1}^{2}$ for Lemma 5.2 so that $\left|\mu_{h_{0}}-\mu\right| \leqq \varepsilon_{1} / 8+2 b \varepsilon_{1}^{2} n+c_{2} \varepsilon_{1}^{2}$.

We note finally that the Beta function has an essentially short memory as the number of scales $h^{\prime}$ above $h$ at which one must know $\vec{v}_{h}$ in order to compute $\vec{v}_{h-1}$ is essentially finite; in fact (see [B.G.P.S.])

$$
\hat{B}_{\mu, M}^{h}\left(\mu_{h} ; \ldots ; \mu_{0}\right)=\hat{B}_{\mu, M}^{h}\left(\mu_{h} ; \ldots ; \mu_{h}\right)+\sum_{k=h+1}^{0} D^{h, k}\left(\mu_{h} ; \ldots ; \mu_{0}\right)
$$

with $\left|D^{h, k}\right|<K \varepsilon^{2} \gamma^{-1 / 2(k-h)}$, if $\left|g_{1, h}\right|,\left|\mu_{h}\right|<\varepsilon$ and $K$ is a constant. But a dynamical system of the form $\mu_{h-1}=\mu_{h}+B\left(\mu_{h}\right)$ with $B$ vanishing at least to the second order cannot have trajectories bounded by a constant unless $B \equiv 0$ (see [B.G.P.S.]). This argument implies Eq. (55).

We return then to the study of Eq. (54): the vanishing of the Beta function Eq. (55) implies that, fixed $v, \delta$ as the above analytic functions in $\lambda$, if $|\lambda-\tilde{\varepsilon} / 2|<$ $\tilde{\varepsilon} / 2$, then $\left|v_{l, h}-v_{l, 0}\right| \leqq c \varepsilon^{2}$ and:

$$
\begin{gathered}
g_{1, h} \rightarrow_{h \rightarrow-\infty} 0 \quad g_{2, h} \rightarrow_{h \rightarrow-\infty} g_{2, \infty} \quad g_{4, h} \rightarrow_{h \rightarrow-\infty} g_{4, \infty} \quad \delta_{h} \rightarrow_{h \rightarrow-\infty} 0 \\
\frac{Z_{h}}{Z_{h-1}} \rightarrow_{h \rightarrow-\infty} \gamma^{-2 \eta},
\end{gathered}
$$

where $g_{2, \infty}, g_{4, \infty}, \eta$ are analytic functions in $\lambda$ for $|\lambda-\tilde{\varepsilon} / 2|<\tilde{\varepsilon} / 2$. From Eq. (49) it is easy to see that $g_{2, \infty}, g_{4, \infty}$ are $O(\varepsilon)$ and $\eta=O\left(\varepsilon^{2}\right)$ as $\eta=c g_{2, \infty}^{2}+O\left(\varepsilon^{2}\right), c>0$.

The 2-points Schwinger function $S(k, k) \equiv S(k)$ is given by

$$
S(k)=\frac{h\left(k_{0}^{2}+E(\vec{k})^{2}\right)}{-i k_{0}+E(\vec{k})}+S \leqq 0(k)\left(1-h\left(k_{0}^{2}+E(\vec{k})^{2}\right)\right),
$$

where $S^{\leqq 0}(k)$ in terms of Fourier transform

$$
\hat{S}^{\leqq 0}(x-y)=\left.\frac{\partial}{\partial \phi^{+}(x) \partial \phi^{-}(y)}\right|_{\phi=0} \log \frac{\mathscr{N}_{0}}{\mathscr{N}} \int P_{Z_{0}}\left(d \psi^{\leqq 0}\right) e^{-V^{0}\left(\sqrt{Z_{0}} \psi\right)+\left(\psi^{+}, \phi^{-}\right)+\left(\phi^{+}, \psi^{-}\right)} .
$$

In order to study $\hat{S}^{\leqq 0}(x-y)$ one shall study a tree expansion similar to that one studied in this and in the preceding section, generated integrating step by step the fields with decreasing frequency. This expansion is described in all details in 
[B.G.P.S.]. The bound Eq. (48) can be easily converted into a bound for the functional derivative of Eq. (73) finding that Schwinger functions are analytic functions of the running couplings and

$$
\hat{S}(x-y)=\sum_{h=-\infty}^{0} \frac{1}{Z_{h}}\left(g^{h}+\varepsilon \bar{g}^{h}\right),
$$

where $\varepsilon$ is supposed small enough and $\left|\bar{g}^{h}(x-y)\right| \leqq \frac{\gamma^{h}}{\left(1+\left(\left(x_{0}\right)_{\pi}^{2}+(\vec{x})_{\pi}^{2}\right)^{N}\right.}$. Of course $S(k)$ is analytic in $\lambda$ if $|\lambda-\varepsilon / 2| \leqq \varepsilon / 2$.

From Eq. (74) it follows that, if $\frac{Z_{h}}{Z_{h-1}} \rightarrow_{h \rightarrow-\infty} \gamma^{-2 \eta}$ then $\hat{S}$ decays, for $T, L \rightarrow$ $\infty,|x-y| \rightarrow \infty$ as $|x-y|^{-1-2 \eta}$. Then, from Eq. (72) and performing the Fourier transform to the two-points Schwinger function we have Theorem 1.2. A simple corollary of this theorem is that the two point Schwinger function $S(x, y)$ behaves, for $|x-y| \rightarrow \infty$, as

$$
\begin{aligned}
S(x, y)= & \int \phi(\vec{k}, x) \phi(-\vec{k}, y) e^{-i k_{0}\left(x_{0}-y_{0}\right)} S(k) \simeq\left(1-A_{0}(\lambda)\right) \frac{S_{0}(x, y)}{|x-y|^{2 \eta(\hat{\imath})}} \\
& +A_{1}(\lambda) \frac{1}{|x-y|^{1+2 \eta(\hat{\imath})}}
\end{aligned}
$$

with $A_{0}(\lambda)$ independent from $x$ and $y$ and with $S_{0}$ being the free pair Schwinger functions.

It remains to discuss the Borel summability. We remember that if for some $\varepsilon>0$ in the domain $|\lambda-\varepsilon / 2| \leqq \varepsilon / 2$ a function $f(\lambda)$ of complex $\lambda$ is such that:

1. $f(\lambda)$ is analytic,

2. $\left|f(\lambda)-\sum_{k=0}^{n} \frac{\lambda^{k}}{k !} f^{k}(0)\right| \leqq C^{n}|\lambda|^{n+1} n !$,

then $f(\lambda)$ is Borel summable in the given domain.

We known that $v_{0}(\lambda)$ and $S(x)$ verifies the first condition. Noting that $\mid \sum_{h=-\infty}^{0}$ $h^{n} \gamma^{h} \mid \leqq n ! C^{n}$ and remembering Eq. (53) an estimate on $v_{h}$ of the form $\left|\frac{\partial^{n} \vec{v}_{h}}{\partial \lambda^{n}}\right| \leqq$ $n ! h^{n} C^{n}$ uniform in $\lambda$ and $h$, which should be clear from the consideration above, seems to be enough to prove Borel summability of $v_{0}(\lambda)$. Without anomalous scaling this would be enough to prove Borel summability for the Schwinger function. But the presence of anomalous scaling requires also that

$$
\left|\frac{\partial^{n} \eta(\lambda)}{\partial \lambda^{n}}\right| \leqq(n !)^{2} C^{n} .
$$

Some cancellations in Eq. (49) strongly support the validity of this estimate, but its rigorous proof requires some extra work.

\section{Conclusions}

In the preceding sections we find that the Fermi surface is anomalous both if the fermions are spinless and the conduction band is not filled or if the fermions are spinning, the interaction repulsive and the band neither filled nor half filled.

Changing the form in the interaction it is possible to have a normal Fermi surface, i.e. $\eta(\lambda) \equiv 0$. We can consider in fact a slightly different model in which 
$\hat{v}_{n}\left(\vec{k}_{1}, \vec{k}_{2}, \vec{k}_{3}, \vec{k}_{4}\right)$ in Eq. (6) is a function with support strictly contained in the region where the $\vec{k}_{i}$ have the same sign and are non-zero. Note that in this case the interaction in the hamiltonian Eq. (1) is not a pair potential, i.e. it has the form $W\left(x_{1}, x_{2}, x_{3}, x_{4}\right)$. Then it is easy to see, by symmetry reasons, that there are no graphs contributing to $g_{1, h}$ or $g_{2, h}$ for every $h$. In fact in the graphs contributing to $g_{1, h}$ or to $g_{2, h}$ there are necessarily vertices in which the incoming or outcoming fields have momenta with different signs. If $g_{2, h}=g_{1, h}=0,\left|v_{l, 0}\right| \leqq \varepsilon, i=4,5$ it follows that $\left|v_{l, h}-v_{l, 0}\right| \leqq c \varepsilon^{2}$ and

$$
g_{4, h} \rightarrow_{h \rightarrow-\infty} g_{4, \infty}, \quad \delta_{h} \rightarrow_{h \rightarrow-\infty} \delta_{\infty}, \quad \frac{Z_{h}}{Z_{h-1}} \rightarrow_{h \rightarrow-\infty} 1,
$$

where $g_{4, \infty}, \delta_{\infty}$ are bounded functions in $v_{0, l}$ and $O(\varepsilon)$. It is not clear if an Hamiltonian with such a potential should be considered the model for some physical situation.

We discuss briefly the cases not covered by Theorems 1.1,1.2. We start from the case in which $p_{F}=\pi / 2 a$. From Sect. (3) we know that in this case there is another relevant running coupling, $g_{3, h}$. In order to study the Beta function at the second order, it is convenient to introduce a new coupling $\tilde{g}_{2, h}=g_{1, h}-2 g_{2, h}$ so that

$$
\begin{aligned}
g_{4, h-1} & =g_{4, h}, \quad g_{3, h-1}=g_{3, h}-\beta g_{3, h} \tilde{g}_{2, h}, \\
\tilde{g}_{2, h-1} & =\tilde{g}_{2, h}, \quad g_{1, h-1}=g_{1, h}-\beta g_{1, h}^{2}, \\
\alpha_{h-1} & =\alpha_{h}+\tilde{\beta}_{1} g_{1, h}^{2}+1 / 4 \tilde{\beta}_{2}\left(g_{1, h}-2 \tilde{g}_{2, h}\right)^{2}+\tilde{\beta}_{3} g_{3, h}^{2}, \\
\zeta^{h-1} & =\zeta^{h}+\tilde{\beta}_{1} g_{1, h}^{2}+1 / 4 \tilde{\beta}_{2}\left(g_{1, h}-2 \tilde{g}_{2, h}\right)^{2}+\tilde{\beta}_{3} g_{3, h}^{2}, \\
v^{h-1} & =\gamma v^{h} .
\end{aligned}
$$

It is possible to choose $\left|v_{i, 0}\right| \geqq \varepsilon$ so that, if $g_{1,0}, \tilde{g}_{2,0} \geqq 0$, then $\tilde{g}_{2, \infty}=\tilde{g}_{2,0}=O(\varepsilon)$ and $g_{3, h}, g_{1, h} \rightarrow h \rightarrow-\infty 0$. The behaviour of the flow at the second order suggests that we try an anomalous scaling. One can repeat the consideration in Sect. (4) and write the analogues of Eq. (49) for the case $p_{F} \neq \pi / 2 a$. However the equations are too difficult and we are not able to prove that the flow is bounded at any order.

Going back to the case $p_{F} \neq \pi / 2 a$ if $g_{1,0}<0$ the running coupling constants exit from the convergence circle of the beta function in finite many steps. The only hope in order to have a bounded flow that can be studied by perturbation theory is that the Beta function have a non-trivial fixed point:

$$
v^{*}=\beta\left(v^{*}\right) \text {. }
$$

Some heuristic consideration [L.E.,S.] and a third order analysis lead to the conjecture that

$$
\begin{gathered}
g_{1, h}, \tilde{g}_{2, h}, g_{3, h} \rightarrow_{h \rightarrow-\infty} g_{1, \infty}^{*}, \tilde{g}_{1, \infty}^{*}, \tilde{g}_{2, \infty}^{*}, g_{3, \infty}^{*}, \quad \delta_{h} \rightarrow_{h \rightarrow-\infty} \delta_{\infty}^{*}, \\
\frac{Z_{h}}{Z_{h-1}} \rightarrow_{h \rightarrow-\infty} \gamma^{-2 \eta^{*}},
\end{gathered}
$$

where $g_{1, h}^{*}, g_{2, h}^{*}, g_{3, h}^{*}, g_{4, h}^{*}, \eta^{*}$ are constants independent from the interaction $\lambda$ and $O(1)$. A similar flow cannot be studied very likely by our perturbative expansion as the radius of convergence is very small. Perhaps one could try to use better 
estimates than those in Sect. (4) or use other techniques in order to enlarge the convergence domain and reach this fixed point (if it exists at all).

Finally we note that, if $p_{F}=\pi / a$, the propagator is given by (if $\overrightarrow{k^{\prime}}=\vec{k}+\pi / a$ ):

$$
\left.g^{h}\left(k^{\prime}\right)=\gamma^{-h} \bar{g}^{h}\left(\gamma^{-h} k_{0}, \gamma^{-h / 2} \overrightarrow{k^{\prime}}\right)=\gamma^{-h} \bar{g}_{I}\left(\gamma^{-h} k_{0}, \gamma^{-h / 2} \overrightarrow{k^{\prime}}\right)+\gamma^{-h / 2} C_{h}\left(\gamma^{-h} k_{0}, \gamma^{-h / 2} \overrightarrow{k^{\prime}}\right)\right)
$$

where

$$
g_{I}\left(\gamma^{-h} k_{0}, \gamma^{-h / 2} \overrightarrow{k^{\prime}}\right)=\frac{f\left(\gamma ^ { 2 } \left(\left[\gamma^{-h} k_{0}\right]^{2}+\left(\beta\left[\gamma^{-h} \overrightarrow{k^{\prime}}\right]^{4}\right)\right.\right.}{-i\left[\gamma^{-h} k_{0}\right]+\tilde{\beta}\left[\gamma^{-h} \overrightarrow{k^{\prime}}\right]^{2}},
$$

and $C_{h}(t)$ weakly dependent on $h, T, L$. One can estimate by a power counting argument like in Sect. (3) the generic graph contributing to $V^{k}$ : in this case one obtains that the size of the graph is bounded by $\gamma^{-k\left(m_{4}+\frac{m_{2}}{2}\right)}$, where $m_{4}$ and $m_{2}$ are the number of the vertex with two or four external lines in the graph. This is the behaviour of not renormalizable field theories. It is unclear whether techniques of [F.G.,F.] for a non-renormalizable field theory can be of any use.

\section{A. Appendix}

We state first some easy consequence of the results in [Ko.], proved in [B.M.]

Lemma A.1. We have

$$
\begin{aligned}
\lim _{\tau \rightarrow n \pi / a^{ \pm}} E\left(\tau+i h_{I}\right) & =\lim _{\tau \rightarrow n \pi / a^{ \pm}} E\left(-\tau+i h_{I}\right), \\
\lim _{\tau \rightarrow n \pi / a^{ \pm}} \phi\left(\tau+i h_{I}, \vec{x}\right) \phi\left(\tau+i h_{I}, \vec{y}\right) & =\lim _{\tau \rightarrow n \pi / a^{ \pm}} \phi\left(-\tau+i h_{I}, \vec{x}\right) \phi\left(-\tau+i h_{I}, \vec{y}\right),
\end{aligned}
$$

where $h_{I} \leqq h_{n-1}$.

Lemma A.2. If $\bar{k}=g+i \bar{h}$ where $\bar{h} \neq h_{n}$ then $\varepsilon(\overline{\vec{k}})=\overline{\vec{k}}^{2}+\varepsilon_{1}(\overline{\vec{k}})$, where $\lim _{g \rightarrow \infty}$ $\frac{\varepsilon_{1}(\bar{k})}{k}=0$, and $|\sqrt{\varepsilon(\bar{k})}-\bar{k}| \leqq \frac{c}{\sqrt{\varepsilon(\vec{k})}}$. Moreover there exist a constant $K$ such that $\phi(\bar{k}, x)<K e^{-\bar{h} a \vec{x}}$ and $\left|\phi(\bar{k}, \vec{x})-e^{i \bar{k} \vec{x}+i \tilde{\varepsilon}(\bar{k}) t}\right| \leqq \frac{K e^{-\bar{h} \vec{x}}}{\sqrt{\varepsilon(\bar{k})}}$.

In order to prove Eq. (15), we start proving the following lemma:

\section{Lemma A.3.}

$$
\begin{gathered}
g_{u . v .}(x, y)=S(x, y)+R_{1}(x, y)+R_{2}(x, y), \\
S(x, y)=H(\vec{x}-\vec{y}) H\left(x_{0}-y_{0}\right) \theta\left(x_{0}-y_{0}\right) \int d \vec{k} \phi(\vec{k},-\vec{x}) \phi(\vec{k}, \vec{y}) e^{-E(\vec{k})\left(x_{0}-y_{0}\right)}, \\
\left|R_{1}(x, y)\right| \leqq \frac{C_{N}}{1+|x-y|^{N}},\left|R_{2}(x, y)\right| \leqq C e^{-a|x-y|},
\end{gathered}
$$

where $E(\vec{k})=\varepsilon(\vec{k})-\mu, N$ is an integer, $H(t)$ is a $C^{\infty}$ compact support function such that $H(t)=1$ for $|t| \leqq 1, H(t)=0$ for $|t| \geqq \gamma, \gamma>1$ and $\theta$ is the step function. 


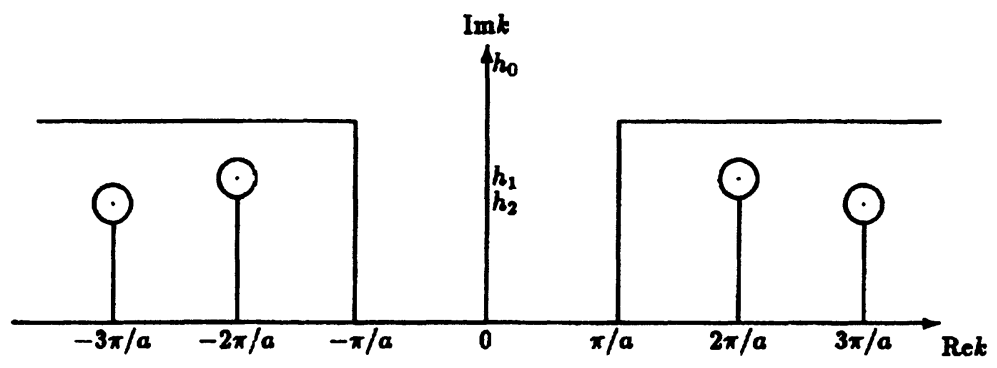

Fig. 2.

Proof. Let $g_{u . v .}(x, y)=g_{1}(x, y)+g_{2}(x, y)$, where in $g_{1}$ the integral is restricted on $k \leqq \pi / a$ and in $g_{2}$ on $k \geqq \pi / a$. It is easy to see that $g_{1}$ belong to $R_{1}$.

For $|\vec{k}| \geqq \pi / a$ we have $E(\vec{k}) \geqq 0$ and $h\left(c^{2}\left(k_{0}^{2}+E(\vec{k})^{2}\right)=1\right.$, so we can write

$$
g_{2}(x, y)=\theta\left(x_{0}-y_{0}\right) \underset{|\vec{k}| \geqq \frac{\pi}{a}}{\int} d \vec{k} e^{-E(\vec{k})\left(x_{0}-y_{0}\right)} \phi(\vec{k},-\vec{x}) \phi(\vec{k}, \vec{y}) .
$$

Let

$$
g_{2}(x, y)=H(\vec{x}-\vec{y}) H\left(x_{0}-y_{0}\right) g_{2}(x, y)+\left(1-H(\vec{x}-\vec{y}) H\left(x_{0}-y_{0}\right)\right) g_{2}(x, y) .
$$

We will show that the second term in Eq. (80) belongs to $R_{2}(x, y)$.

It is always possible to choose $\bar{h} \neq h_{n}, \bar{h}<h_{0}$ such that $\operatorname{ReE}(g+i \bar{h})>0$ for $|g| \geqq \pi / a$. Let us remember that $\lim _{n \rightarrow \infty} h_{n}=0$. We can shift the integral of $g_{2}(x, y)$ to a line with a complex part $\bar{h}$ considering the following integral in the complex plane:

$$
\int_{\gamma_{1}} \phi(k,-\vec{x}) \phi(k, \vec{y}) e^{-E(k)\left(x_{0}-y_{0}\right)},
$$

where $\gamma_{1}$ is a connected path such that the function is analytic in its interior and $-[\tilde{n}+1 / 2] \pi / a \leqq \operatorname{Re} k \leqq[\tilde{n}+1 / 2] \pi / a, \tilde{n} \rightarrow \infty$ (see the picture)

We can eliminate the integral along the part of the path perpendicular to the real axis using periodicity Eq. (78). The integrals on the circle around the nonanalyticity points $\vec{k}_{n}$ give a vanishing contribution when the radius is sent to zero by Eq. (3), (2).

Since in the limit $\tilde{n} \rightarrow \infty$ the integral on the path from $\tilde{n}+1 / 2$ and $(\tilde{n}+1 / 2)+$ $i \bar{h}$ is vanishing we have

$$
\begin{aligned}
\int_{|\vec{k}|>\pi / a} d \vec{k} \phi(\vec{k}, \vec{x}) \phi(-\vec{k}, \vec{y}) e^{-E(\vec{k})\left(x_{0}-y_{0}\right)}= & \int_{|\vec{k}|>\pi / a} d \vec{k} \phi(\vec{k}+i \bar{h}, \vec{x}) \phi(-\vec{k}-i \bar{h}, \vec{y}) \\
& e^{-E(\vec{k}+i \bar{h})\left(x_{0}-y_{0}\right)}
\end{aligned}
$$

so that, using the properties of Block wave listed in Lemma A.2, we have that $g_{2}$ belong to $R_{2}$. Finally summing and subtracting:

$$
H(\vec{x}-\vec{y}) H\left(x_{0}-y_{0}\right) \theta\left(x_{0}-y_{0}\right) \int_{|\vec{k}| \leqq \pi / a} d \vec{k} \phi(\vec{k}, \vec{x}) \phi(\vec{k},-\vec{y}) e^{-E(\vec{k})\left(x_{0}-y_{0}\right)},
$$

and noting that it belongs to $R_{1}(x, y)$, we have the lemma. Q.E.D. 


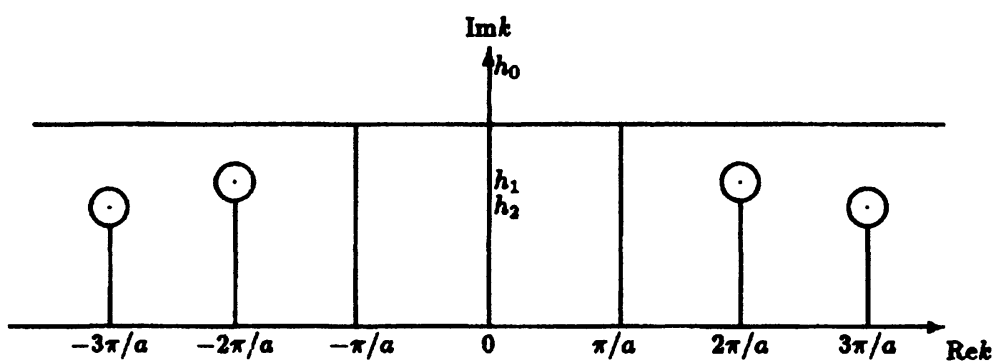

Fig. 3.

We regularize $S(x, y)$ replacing in Eq. (79) $\theta\left(x_{0}-y_{0}\right)$ by $\theta_{N}\left(x_{0}-y_{0}\right)$, where $\theta_{N}(t)$ is a smooth function with support in $\left(\gamma^{-N}, \gamma\right)$, where $N$ is a positive integer. $\theta_{N}(t)$ can be written as: $\theta_{N}(t)=\sum_{i}^{N} f\left(\gamma^{l} t\right)$ with $f(t)=(H(t / \gamma)-H(t)) \theta(t)$, so that $\theta(t) h(t)=\lim _{N \rightarrow \infty} \theta_{N}(t), \lim _{N \rightarrow \infty} S_{N}(x, y)=S(x, y)$. We write $S_{N}(x, y)=$ $\sum_{h=1}^{N} C_{h}(x, y)$, where

$$
C_{h}(x, y)=H(\vec{x}-\vec{y}) f\left(\gamma^{h}\left(x_{0}-y_{0}\right)\right) \int d \vec{k} \phi(\vec{k}, \vec{x}) \phi(\vec{k},-\vec{y}) e^{-E(\vec{k})\left(x_{0}-y_{0}\right)} .
$$

Lemma A.4. $C_{h}(x, y)$ can be written in the following way:

$$
\begin{aligned}
& C_{h}(x, y)=\gamma^{h / 2} C_{1, h}\left(\gamma^{h}\left(x_{0}-y_{0}\right), \gamma^{h / 2}(\vec{x}-\vec{y})\right)+C_{2, h}\left(\gamma^{h} x_{0}, \gamma^{h / 2} \vec{x} ; \gamma^{h} y_{0}, \gamma^{h} \vec{y}\right), \\
& \gamma^{h / 2} C_{1, h}\left(\gamma^{h}\left(x_{0}-y_{0}\right), \gamma^{h / 2}(\vec{x}-\vec{y})\right)= H(\vec{x}-\vec{y}) f\left(\gamma^{h}\left(x_{0}-y_{0}\right)\right) \gamma^{h / 2} \\
& \times \int d k e^{-i \vec{k} \gamma^{h / 2}(\vec{x}-\vec{y})} e^{-\left(\vec{k}^{2} \gamma^{h}-\mu\right)\left(x_{0}-y_{0}\right)},
\end{aligned}
$$

and $C_{1, h}, C_{2, h}$ are smooth functions such that $\left|C_{1, h}(x-y)\right|,\left|C_{2, h}\left(x_{0}, x ; y_{0}, y\right)\right| \leqq$ $C e^{-a|x-y|}$.

Proof. If $\vec{x}=u+n_{x} a, \vec{y}=v+n_{y} a$, we write

$$
\begin{gathered}
\phi(\vec{k}+i \bar{h}, \vec{x})=e^{i(\vec{k}+i \bar{h}) \vec{x}+i \tilde{\varepsilon}(\vec{k}+i \bar{h}) u}+\phi_{1}(\vec{k}+i \bar{h}, \vec{x}), \\
\bar{\varepsilon}(\vec{k}+i \bar{h})=(\vec{k}+i \bar{h})^{2}=(\vec{k}+i \bar{h})^{2}+\varepsilon_{1}(\vec{k}), \quad \sqrt{\bar{\varepsilon}(\vec{k}+i \bar{h})}=(\vec{k}+i \bar{h})+\tilde{\varepsilon}(\vec{k}+i \bar{h}) .
\end{gathered}
$$

We can shift the integral of $C_{2, h}$ to a line with imaginary part $\gamma^{h / 2} \bar{h}, \bar{h}>\max _{n} h_{n}$ using a connected path $\gamma_{2}$ (see the picture)

We have

$$
C_{2, h}\left(\gamma^{h} x_{0}, \gamma^{h / 2} \vec{x}, \gamma^{h} y_{0}, \gamma^{h} \vec{y}\right)=H(\vec{x}-\vec{y}) f\left(\gamma^{h}\left(x_{0}-y_{0}\right)\right)
$$

$\int_{0}^{1} d \sigma \int d k \frac{\partial}{\partial \gamma^{-h / 2} \sigma}\left[e^{i(\vec{k}+i \bar{h}) \vec{y} \gamma^{h / 2}+l\left[\sigma \gamma^{-h / 2}\right] \tilde{\varepsilon}\left(\gamma^{h / 2}(k+i \bar{h}) \gamma^{h / 2} \vec{y}\right.}+\sigma \gamma^{-h / 2}\left(\phi_{1}\left(\gamma^{h / 2}(\vec{k}+i \bar{h}), \vec{y}\right) \gamma^{h / 2}\right)\right]$

$$
\begin{gathered}
{\left[e^{-l(\vec{k}+i \bar{h}) \vec{x} \gamma^{h / 2}-i\left[\sigma \gamma^{-h / 2}\right] \tilde{e}\left(\gamma^{h / 2}(k+i \bar{h})\right) \gamma^{h / 2} \vec{x}}+\sigma \gamma^{-h / 2}\left(\phi_{1}\left(\gamma^{h / 2}(\vec{k}+i \bar{h}), \vec{x}\right) \gamma^{h / 2}\right)\right]} \\
e^{-\left[(\vec{k}+i \bar{h})^{2}+\left(\sigma \gamma^{-h / 2}\right) \gamma^{-h / 2} \varepsilon_{1}\left(\gamma^{h / 2}(\vec{k}+i \bar{h})\right)\right] \gamma^{h}\left(x_{0}-y_{0}\right)} .
\end{gathered}
$$


The lemma is proved noting that, from Lemma A.2:

$$
\lim _{\vec{k} \rightarrow \infty}\left|\vec{k} \phi_{1}(\vec{k}+i \bar{h}, \vec{x})\right|=e^{-\bar{h} \vec{x}} C \quad \lim _{\vec{k} \rightarrow \infty} \frac{\varepsilon_{1}(\vec{k}+i \bar{h})}{\vec{k}}=0 \quad \lim _{\vec{k} \rightarrow \infty} \tilde{\varepsilon}(\vec{k}+i \bar{h}) \vec{k}=\text { const }
$$

Q.E.D.

Summing over $C_{h}$ and making the limit $N \rightarrow \infty$ we have Eq. (16).

\section{B. Appendix 2}

Consider $V^{k}\left(\tau, P_{v_{0}}, x_{v_{0}}\right)$ on each vertex of $\tau$ with the action of $\mathscr{R}$ given by Eq. (32) and analogues; if the delta-functions are not integrated away there are no zeros or interpolated points. In order to obtain Eq. (43) we write the renormalization using Eq. (33) by integrating the delta-functions. We start from the first (climbing the tree from the root) non-trivial vertex $v$ in which the action $\mathscr{R} \neq 1$. The renormalization produces a zero $(x-y)^{x}$, if $z=1,2$ and $x, y$ are points in the cluster $v$, that we can write as

$$
(x-y)^{x}=\left(\sum_{l, j, i^{\prime}, j^{\prime}}\left(x_{i, j}-x_{i^{\prime}, j^{\prime}}\right)\right)^{z}
$$

Where $x_{i, j}-x_{i^{\prime}, J^{\prime}}$ are defined in the following way:

1. $x_{l, j}, x_{i^{\prime}, J^{\prime}}$ is the argument of a propagator belonging to $\tilde{T}_{v}$,

2. otherwise $x_{i, j}, x_{i^{\prime}, J^{\prime}}$ are the coordinates of some field with label in the set $P_{v_{i}}$ if $v_{l}$ is the frequency label of the generic subtrees coming from $v$.

If $\left|P_{v_{l}}\right|$ is equal to 2 or 4 the renormalization, acting on $v_{l}$, can produce some other factor $\left(x_{i, j}-x_{l^{\prime}, j^{\prime}}\right)^{z}$. However this does not happen. In fact if we call the effective potential on which $\mathscr{R}$ act in a non-trivial way as $V^{h_{v_{i}}}\left(\tau_{v_{l}}, P_{v_{l}} ; x_{1}-x_{2}, x_{2}-\right.$ $\left.x_{3}, x_{3}-x_{4}\right)$ or $V^{h_{l}}\left(\tau_{v_{l}}, P_{v_{l}} ; x_{1}-x_{2}\right)$ we have

$$
\begin{aligned}
& \int_{\Lambda}\left(\prod_{i=1} d x_{l}\right)\left(x_{l}-x_{j}\right)^{\alpha} \mathscr{R} V^{h_{v_{i}}}\left(\tau_{v_{l}}, P_{v_{l}} ; x_{1}-x_{4}, x_{2}-x_{4}, x_{3}-x_{4}\right) \\
& \quad \times \hat{\psi}_{x_{1}, \vec{\omega}_{1}, \sigma}^{+} \hat{\psi}_{x_{2}, \vec{\omega}_{2}, \sigma^{\prime}}^{+} \hat{\psi}_{x_{3}, \vec{\omega}_{3}, \sigma^{\prime}}^{-} \hat{\psi}_{x_{4}, \vec{\omega}_{4}, \sigma}^{-}= \\
& \int_{\Lambda}\left(\prod_{l=1} d x_{i}\right)\left(x_{l}-x_{J}\right)^{\alpha} V^{h_{v_{i}}}\left(\tau_{v_{i}}, P_{v_{l}} ; x_{1}-x_{4}, x_{2}-x_{4}, x_{3}-x_{4}\right) \\
& \quad \times \hat{\psi}_{x_{1}, \vec{\omega}_{1}, \sigma}^{+} \hat{\psi}_{x_{2}, \vec{\omega}_{2}, \sigma^{\prime}}^{+} \hat{\psi}_{x_{3}, \vec{\omega}_{3}, \sigma^{\prime}}^{-} \hat{\psi}_{x_{4}, \vec{\omega}_{4}, \sigma}^{-}, \\
& \int_{\Lambda} d x_{1} d x_{2}\left(x_{1}-x_{2}\right)^{\beta} \hat{\psi}_{x_{1}, \vec{\omega}, \sigma}^{+} \hat{\psi}_{x_{2}, \vec{\omega}, \sigma}^{-} \mathscr{R} V^{h_{v_{i}}}\left(\tau_{v_{l}}, P_{v_{l}} ; x_{1}-x_{2}\right)= \\
& \left.\int_{\Lambda} d x_{1} d x_{2}\left(x_{1}-x_{2}\right)^{\beta} \hat{\psi}_{x_{1}, \vec{\omega}}^{+}\left(\vec{\psi}_{1}-\vec{\psi}_{x_{2}, \vec{\omega}}^{-} V^{h_{v_{i}}}\right) \hat{\psi}_{x_{1}, \vec{\omega}, \sigma}^{+} \hat{\psi}_{v_{v_{i}}, \vec{\omega}, \sigma}^{-}, P_{v_{i}} ; x-y\right), \\
& \int_{\Lambda} d x_{1} d x_{2}\left(\vec{x}_{1}-\vec{x}_{2}\right) \hat{\psi}_{x_{1}, \vec{\omega}, \sigma}^{+} \hat{\psi}_{x_{2}, \vec{\omega}, \sigma}^{-}
\end{aligned}
$$




$$
\begin{aligned}
& \times V^{h_{i_{i}}}\left(\tau_{v_{l}}, P_{v_{t}} ; x_{1}-x_{2}\right)-\int_{\Lambda} d t V^{h_{l_{l}}}\left(\tau_{v_{l}}, P_{v_{i}} ; t\right) \frac{L}{\pi} \sin \left(\vec{t} \frac{\pi}{L}\right) \cos \frac{\pi}{\beta} t_{0} \\
& \times \int_{\Lambda} d x_{1} d x_{2} \hat{\psi}_{x_{1}, \vec{\omega}, \sigma}^{+} \hat{\psi}_{x_{2}, \vec{\omega}, \sigma}^{-} \delta\left(x_{1}-x_{2}\right)- \\
& \int_{\Lambda} d x_{1} d x_{2} \hat{\psi}_{x_{1}, \vec{\omega}, \sigma}^{+} \vec{\omega} \Delta \psi_{x_{1}, \vec{\omega}, \sigma}\left(\vec{x}_{1}-\vec{x}_{2}\right) V^{h_{l_{l}}}\left(\tau_{v_{l}}, P_{v_{l}} ; x_{1}-x_{2}\right) \\
& \times \frac{L}{\pi} \sin \left(\vec{\omega}\left(\vec{x}_{1}-\vec{x}_{2}\right) \frac{\pi}{L} \cos \frac{i \pi}{\beta}\left(x_{1,0}-x_{2,0}\right),\right. \\
& \int_{\Lambda} d x_{1} d x_{2}\left(x_{1,0}-x_{2,0}\right) \hat{\psi}_{x_{1}, \vec{\omega}}^{+} \hat{\psi}_{x_{2}, \vec{\omega}}^{-} \mathscr{R} V^{h_{l_{l}}}\left(\tau_{v_{l}}, P_{v_{l}} ; x_{1}-x_{2}\right)= \\
& \quad \int_{\Lambda} d x_{1} d x_{2}\left(x_{1,0}-x_{2,0}\right) \hat{\psi}_{x_{1}, \vec{\omega}, \sigma}^{+} \hat{\psi}_{x_{2}, \vec{\omega}, \sigma}^{-} V^{h_{v_{i}}}\left(\tau_{v_{l}}, P_{v_{l}} ; x_{1}-x_{2}\right) \\
& -\int_{\Lambda} d t V^{h_{l_{l}}}\left(\tau_{v_{l}}, P_{v_{l}} ; t\right) \frac{\beta}{\pi} \sin \left(t_{0} \frac{\pi}{\beta}\right) \cos \left(\frac{\pi}{L} \vec{t}\right) \\
& \quad \times \int_{\Lambda} d x_{1} d x_{2} \hat{\psi}_{x_{1}, \vec{\omega}, \sigma}^{+} \hat{\psi}_{x_{2}, \vec{\omega}, \sigma}^{-} \delta\left(x_{1}-x_{2}\right)
\end{aligned}
$$

where $\beta>1$ and $A=\bar{\partial}-\partial$. The renormalization of the subtrees does not produce any factor $\left(x_{l}-x_{j}\right)$ or $(x-y)$ in Eq. $(85),(86)$ or only a factor $(x-y)$ in Eq. $(87),(88)$. We can then repeat for the subtree $v_{l}$ the considerations made for the cluster $v$, writing $\left(x_{l}-y_{l}\right)$ or $(x-y)$ like in Eq. (84). Of course if in some subtree with frequency $v_{l}$, for some choice of $P_{v_{l}}$ the renormalization acts in a non-trivial way, integrating the corresponding deltas and using Eq. (35), the arguments of the propagators which connect $v_{1} \ldots, v_{s}$ form a tree $T_{v}$ which joins simple or interpolated points.

Iterating this argument for all vertices $v$ we have Eq. (43).

\section{Appendix 3}

We will give here a sketch of the proof. More detail can be found in [B.M.]. We want to prove that

$$
\prod_{l=1}^{n} d x_{i}=\prod_{i=1}^{n-1} d y_{l} d x_{1},
$$

where $x_{1}$ is the root of the tree.

If we consider a minimal cluster, i.e. a cluster containing only points, there is no interpolated point inside it, so we have $\prod_{i} d x_{l}^{(j)}=d x_{1}^{(j)} \prod d y_{l}^{(j)}$, where $j$ is the cluster index and $x_{l}^{(J)}, y_{l}^{(J)}$ are points and lines internal to cluster $j$. We can now write

$$
\prod_{l=1}^{n} d x_{l}=\prod_{j, l} d y_{l, j} \prod_{j} d x_{1, j} .
$$

Let $\bar{l}$ be the line connecting the cluster 1 to cluster 2 . We have $y_{\bar{l}}=x_{1}^{\prime}-x_{2}^{\prime}$ with $x_{j}^{\prime}=\sum_{l} \lambda_{l, j} x_{l, j}$ for suitable interpolating parameters $\lambda_{l}^{j}\left(\sum_{l} \lambda_{l, j}=1\right.$ see Sect. 4$)$. So we have

$$
\begin{aligned}
x_{1,2} & =x_{1,2}+y_{l}-x_{2}^{\prime}+x_{1}^{\prime} \\
& =\sum_{l} \lambda_{l, 2}\left(x_{1,2}-x_{l, 2}\right)+y_{l}+x_{1}^{\prime} .
\end{aligned}
$$


Now $x_{1,2}-x_{i, 2}$ can be written in terms of $y_{l, 2}$ and $x_{1}^{\prime}$ in terms of $y_{l, 1}$ and $x_{1,1}$ so that we can fix $x_{1,1}$ and substitute $d x_{1,2}$ by $d y_{\bar{l}}$ in Eq. (90). By using this fact we can make the change of variables for the line connecting the minimal clusters. Clearly we can go on recursively on the level of the clusters and reach all the graphs.

Acknowledgements. We are deeply indebted with G.Benfatto and G.Gallavotti for suggesting to us the subject of this work and for their continuous and encouraging advice. Particular thanks to G.Benfatto for granting us access to his personal notes. We also sincerely thank A.Neves and B.Scoppola for many suggestions and discussions.

\section{References}

[B.G.] Benfatto, G., Gallavotti, G.: Perturbation theory of the Fermi surface in a quantum liquid. A general quasiparticle formalism and one dimensional system. Stat. Phys. 59, 541-664 (1990)

[B.G.M.] Benfatto, G., Gallavotti, G., Mastropietro, V.: Renormalization theory and the Fermi surface in the Luttinger model. Phys. Rev. B 45,5468-5480 (1992)

[B.G.P.S.] Benfatto, G., Gallavotti, G., Procacci, A., Scoppola, B.: Beta function and Schwinger functions for a many fermions system in one dimension. Anomaly of the Fermi surface. Commun Math. Phys. 160, 93-171 (1994)

[B.M.] Bonetto, F., Mastropietro, V.: Renormalization group theory in a $\mathrm{d}=1$ system of interacting fermions in a periodic potential. CARR preprint 25/93

[D.M.] Di Castro, C., Metsner, W.: Conservation laws and correlation functions in the Luttinger liquids. Phys. Rev.

[F.] Felder, G.: Construction of a non-trivial planar field theory with an ultraviolet stable fixed point. Commun. Math. Phys. 102, 139-155 (1985)

[F.G.] Felder, G., Gallavotti, G.: Perturbation theory and Non-Renormanizable scalar fields. Commun. Math. Phys. 102, 549-571 (1985)

[G.] Gallavotti, G.: Renormalization theory and the ultraviolet stability for scalar fields via renormalization groups methods. Rev. Mod. Phys. 57, 471-562 (1985)

[G.Sc.] Gentile, G., Scoppola, B.: Renormalization group and the ultraviolet problem in the Luttinger model. Commun. Math. Phys. 154, 135-179 (1993)

[K.] Kohn, W.: Analytic properties of Bloch waves and Wannier function. Phys. Rev. 115, 4, 809-821 (1959)

[L.] Luttinger, J.: An exactly soluble model of a many fermions system. J. Math. Phys. 4, 1154-1162 (1963)

[Le.] Lesniewski, A.: Effective action for the Yukawa 2 quantum field. Theory. Commun. Math. Phys. 108, 437-467 (1987)

[L.E.] Luther, A., Emery, E.: Backward scattering in the one dimensional electron gas. Phys. Rev. Lett. 33, (1974)

[M.] Mattis, D.: Band theory of magnetism in metals in context of exactly soluble models. Physics 1, 183-193 (1964)

[M.L] Mattis, D., Lieb, E.: Exact solution of a many fermion system and its associated boson field. J. Math. Phys. 6, 304 (1965)

[Ma.1] Mastropietro, V.: Interacting soluble Fermi systems in one dimension. Nuovo Cimento 109B, 1-24 (1994)

[S.] Solyom, J.: The Fermi gas model of one dimensional conductors. Adv. Phys. 28, 201-303 (1958)

[Sh] Shankar, R.: Renormalization group approach to interacting fermion 
